Florida International University FIU Digital Commons

\title{
Multi-Resolution Modeling of Managed Lanes with Consideration of Autonomous/Connected Vehicles
}

Somaye Fakharian Qom

Florida International University, sfakh002@fiu.edu

DOI: $10.25148 /$ etd.FIDC000762

Follow this and additional works at: https://digitalcommons.fiu.edu/etd

Part of the Transportation Engineering Commons

\section{Recommended Citation}

Fakharian Qom, Somaye, "Multi-Resolution Modeling of Managed Lanes with Consideration of Autonomous/Connected Vehicles" (2016). FIU Electronic Theses and Dissertations. 2559.

https://digitalcommons.fiu.edu/etd/2559 


\title{
FLORIDA INTERNATIONAL UNIVERSITY
}

Miami, Florida

\section{MULTI-RESOLUTION MODELING OF MANAGED LANES WITH CONSIDERATION OF AUTONOMOUS/CONNECTED VEHICLES}

\author{
A dissertation submitted in partial fulfillment of \\ the requirements for the degree of \\ DOCTOR OF PHILOSOPHY \\ in \\ CIVIL ENGINEERING
}

by

Somaye Fakharian Qom 
To: Interim Dean Ranu Jung

College of Engineering and Computing

This dissertation, written by Somaye Fakharian Qom, and entitled Multi-Resolution Modeling of Managed Lanes with Consideration of Autonomous/Connected Vehicles, having been approved in respect to style and intellectual content, is referred to you for judgment.

We have read this dissertation and recommend that it be approved.

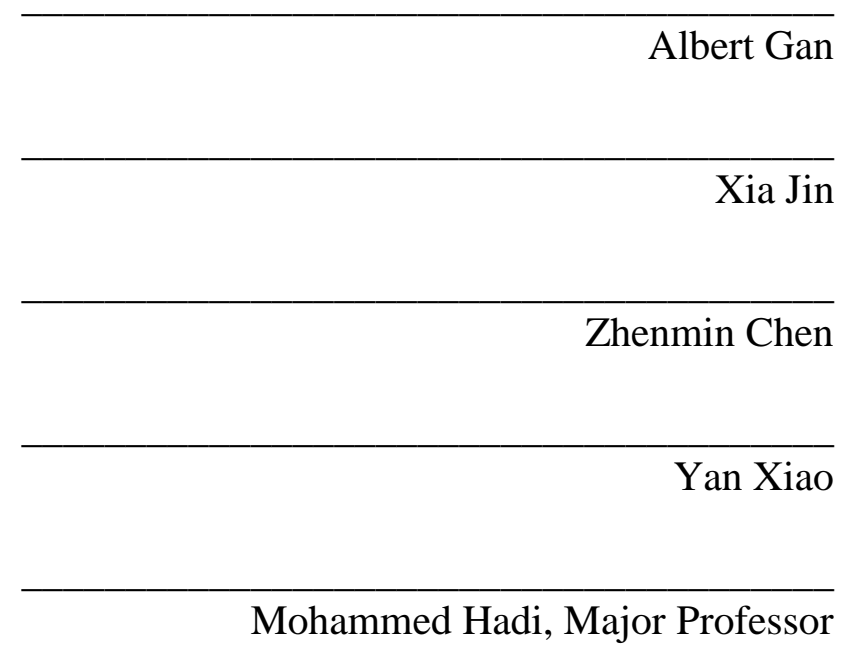

Date of Defense: June 29, 2016

The dissertation of Somaye Fakharian Qom is approved.

Interim Dean Ranu Jung College of Engineering and Computing

Andrés G. Gil Vice President for Research and Economic Development and Dean of the University Graduate School

Florida International University, 2016 
(C) Copyright 2016 by Somaye Fakharian Qom

All rights reserved. 


\section{DEDICATION}

I dedicate this dissertation to my beloved parents, Masoume and Morteza, and my lovely sister, Samira, for their unconditional love, their endless support, and encouragement. 


\section{ACKNOWLEDGMENTS}

First and foremost, I would like to strongly thank my advisor, Dr. Mohammed Hadi, for his guidance, patience, and for his confidence in me. It was my honor to work with such a well-respected advisor in the Intelligent Transportation Systems (ITS) field. He was generous with the time he spent on advising me and training me to conduct high quality research, and to develop a variety of skills that are necessary to succeed in my professional career.

I experienced a great teamwork environment in the Integrated Intelligent Transportation Systems (IITS) research lab. I would like to extend my deep appreciation extend my appreciation to Dr. Yan Xiao, who is incredibly knowledgeable and kind to share her knowledge. I appreciate her continued support of me in different endeavors, including leading student chapters at Florida International University. I would also like to thank my friend, Prof. Hani Mahmassani, for his valuable advice on professional and academic development.

I am also grateful to my committee members, Dr. Albert Gan, Dr. Zhenmin Chen, Dr. Xia Jin, and Dr. Yan Xiao, for serving on my committee, and for their valuable comments, suggestions, and interest in my research.

I will be always thankful to my dear friends Mohamadreza, Paniz, Shagahyegh, Fateme, and Maneli for their assistance, friendship, continued support, care, and encouragement. 


\title{
ABSTRACT OF THE DISSERTATION \\ MULTI-RESOLUTION MODELING OF MANAGED LANES WITH \\ CONSIDERATION OF AUTONOMOUS/CONNECTED VEHICLES
}

\author{
by
}

Somaye Fakharian Qom

Florida International University, 2016

Miami, Florida

\section{Professor Mohammed Hadi, Major Professor}

Advanced modeling tools and methods are essential components for the analyses of congested conditions and advanced Intelligent Transportation Systems (ITS) strategies such as Managed Lanes (ML). A number of tools with different analysis resolution levels have been used to assess these strategies. These tools can be classified as sketch planning, macroscopic simulation, mesoscopic simulation, microscopic simulation, static traffic assignment, and dynamic traffic assignment tools. Due to the complexity of the managed lane modeling process, this dissertation investigated a Multi-Resolution Modeling (MRM) approach that combines a number of these tools for more efficient and accurate assessment of ML deployments.

This study clearly demonstrated the differences in the accuracy of the results produced by the traffic flow models incorporated into different tools when compared with real-world measurements. This difference in the accuracy highlighted the importance of the selection of the appropriate analysis levels and tools that can better estimate ML and General Purpose Lanes (GPL) performance. The results also showed the importance of calibrating traffic flow model parameters, demand matrices, and assignment parameters 
based on real-world measurements to ensure accurate forecasts of real-world traffic conditions. In addition, the results indicated that the real-world utilization of ML by travelers can be best predicated with the use of dynamic traffic assignment modeling that incorporates travel time, toll, and travel time reliability of alternative paths in the assignment objective function. The replication of the specific dynamic pricing algorithm used in the real-world in the modeling process was also found to provide better forecast of ML utilization.

With regards to Connected Vehicle (CV) operations on ML, this study demonstrated the benefits of using results from tools with different modeling resolution to support each other's analyses. In general, the results showed that providing toll incentives for Cooperative Adaptive Cruise Control (CACC)-equipped vehicles to use ML is not beneficial at lower market penetrations of CACC due to the small increase in capacity with these market penetrations. However, such incentives were found to be beneficial at higher market penetrations, particularly with higher demand levels. 
1. INTRODUCTION

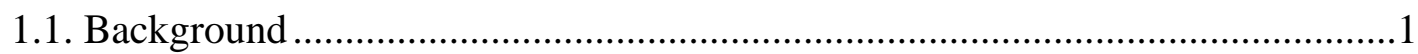

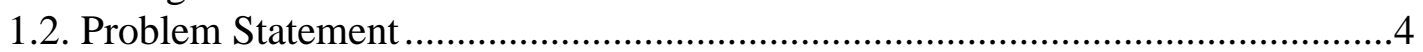

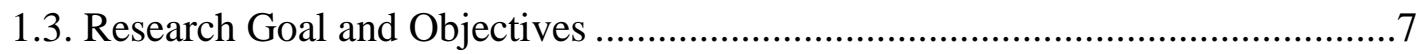

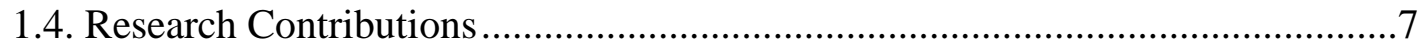

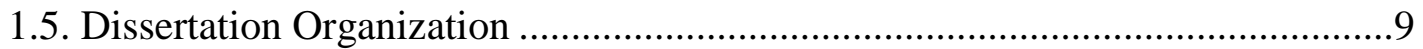

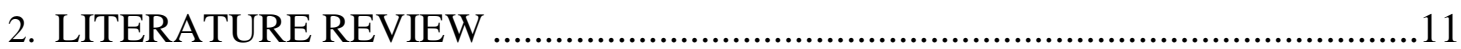

2.1. Multi-Resolution Modeling (MRM) ...........................................................11

2.1.1. Review of Simulation and Dynamic Traffic Assignment (DTA) Tools......12

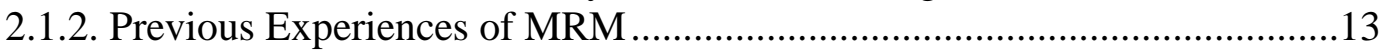

2.2. Managed Lane (ML) Modeling Structure ....................................................15

2.2.1. Sketch Planning and Demand Model-Based Tools ....................................16

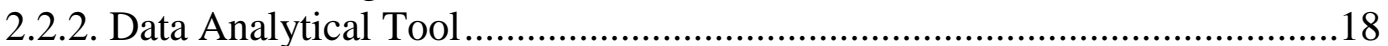

2.2.3. ML Modeling Input Parameters.............................................................19

2.2.4. Dynamic Traffic Assignment (DTA) Implementation in ML Modeling.....21

2.3. Mobility Impacts of Advanced Vehicle Technology ............................................23

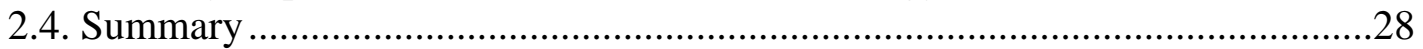

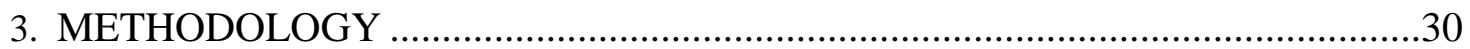

3.1. Overview of Developed MRM Framework .......................................................

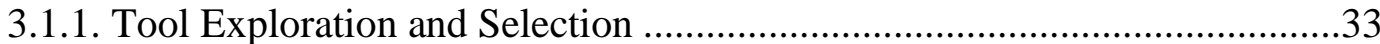

3.1.2. Data Sources and Pre-Processing..................................................................

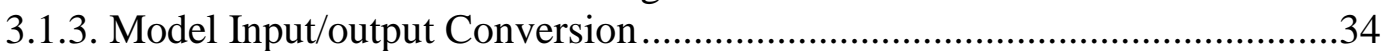

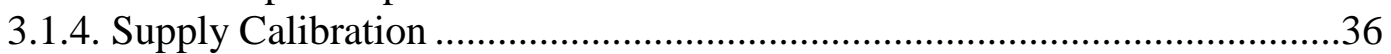

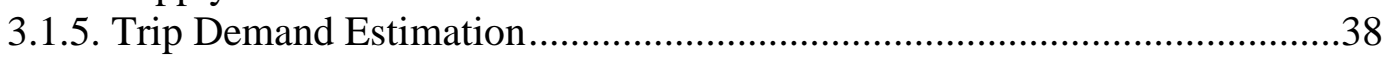

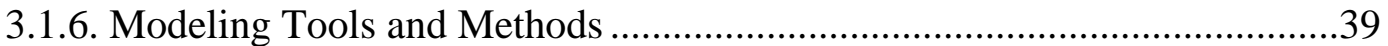

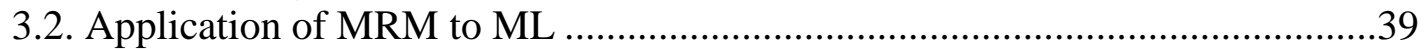

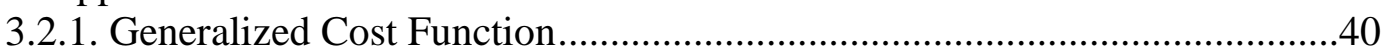

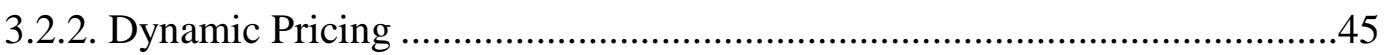

3.3. Assessing the Impacts of Advanced Vehicle Technology Utilizing MRM to

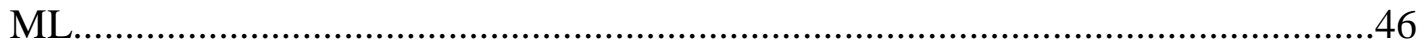

3.3.1. Capacity Impact Estimation based on Microscopic Simulation ...................46

3.3.2. Modeling of CACC Vehicle on Managed Lanes Based on Macroscopic

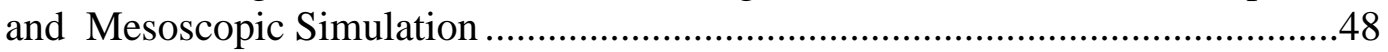

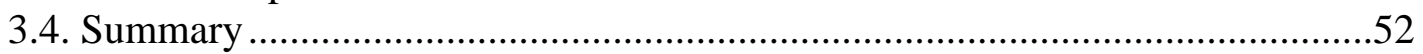

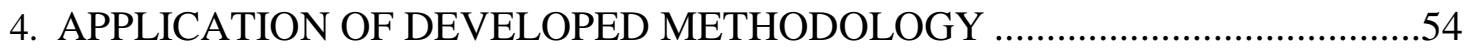

4.1. Data Processing and Importing to DTA Tools ................................................54

4.1.1. Network and Demand Data Conversion .................................................55 


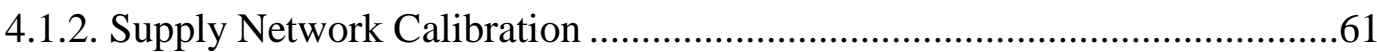

4.2. Origin-Destination Matrix Estimation (ODME) .............................................68

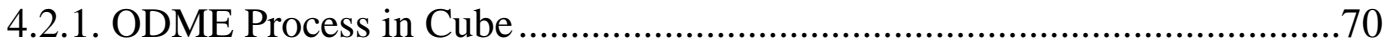

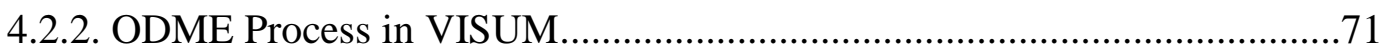

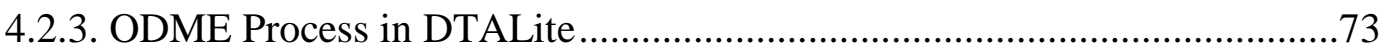

4.2.4. Summary of Tool Assessment for Demand Estimation................................75

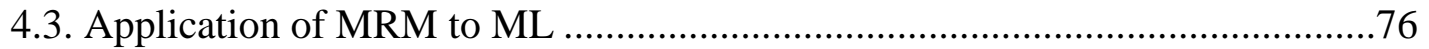

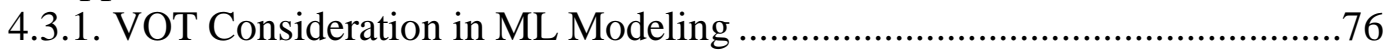

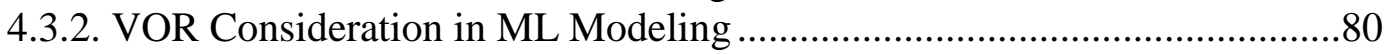

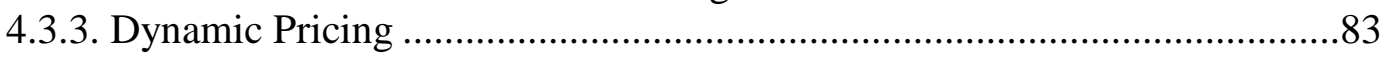

4.4. Modeling of Cooperative Adaptive Cruise Control (CACC) in ML Utilizing

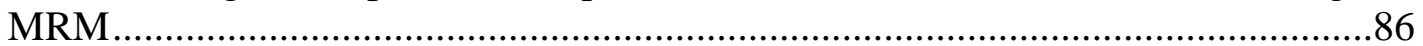

4.4.1. Capacity Impact Estimation based on Microscopic Simulation ...................86

4.4.2. Modeling the Impacts of CACC Vehicle Based on Macroscopic and

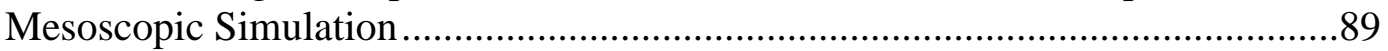

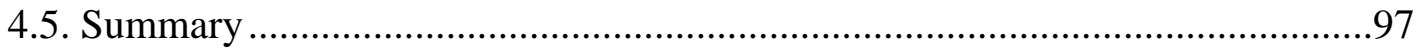

5. CONCLUSIONS AND RECOMMENDATIONS ………......................................

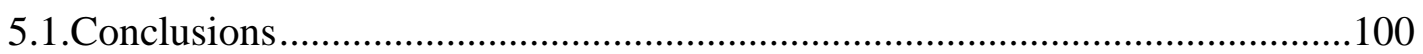

5.2. Recommendations for Future Works ...............................................................105

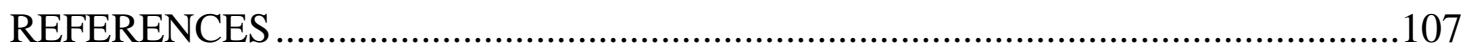

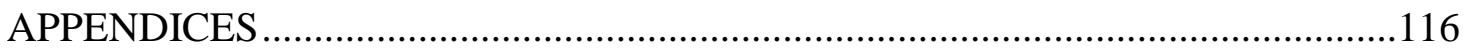

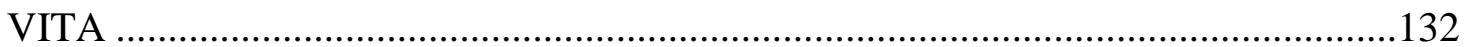




\section{LIST OF TABLES}

TABLE

PAGE

Table 3-1 Coefficients for Different TTI Percentile (Hadi et al., 2014)

Table 3-2 Simulation Results for Different Percentage of ACC (Shladover et al., 2012).47

Table 3-3 Simulation Results for Different Percentage of CACC

(Shladover et al., 2012)

Table 3-4 Estimation of Lane Capacity for ACC and CACC Vehicles at Time Gaps Chosen by Drivers in Field Data with the remaining vehicles manually driven (Shladover et al., 2012)

Table 4-1 Required Link Type to Model Traffic Network in DTALite .56

Table 4-2 Performance Measures for TFM Calibration Results Using Different DTA

Tools .65

Table 4-3 Goodness of Fit for Simulated Volume Based on Factorized Demand Matrix.75

Table 4-4 Goodness of Fit for Simulated Volume Based on Calibrated Demand Matrix.76

Table 4-5 Real-World Toll Value (\$) for I-95 Northbound in April, 2015 .......................78

Table 4-6 Diverted Volumes (veh/ln/15min) to ML for Different VOTs ........................79

Table 4-7 Goodness of Fit Statistics for Diverted Volume Replication Based on Different VOT. .80

Table 4-8 Goodness-of-Fit Statistics for Diverted Volume Replication with and without the Consideration of VOR

Table 4-9 I-95 ML Old Toll Policy (Before March, 2014)..........................................83

Table 4-10 I-95 ML New Toll Policy (After March, 2014) ............................................84

Table 4-11 Variation of Percentage of ML Share with Respect to toll Scheduled Policy Utilizing Different Tools (\%)... 


\section{LIST OF FIGURES}

FIGURE

PAGE

Figure 1-1 Examples of MRM Structure (FHWA, 2013a) ............................................

Figure 2-1 Example of Modeling Structures (FHWA, 2012a) .......................................13

Figure 2-2 Modeling Framework for Mesoscopic-Microscopic Integration

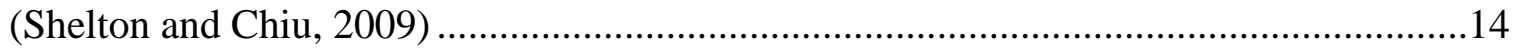

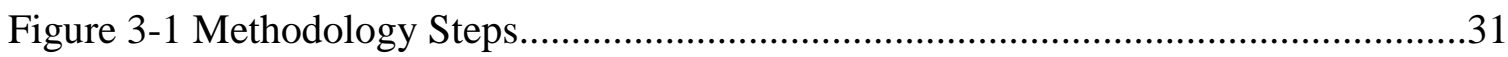

Figure 3-2 Development of General Multi-Resolution Modeling (MRM) Framework ....32

Figure 3-3 Multi-Resolution Modeling (MRM) for Managed Lanes (ML) ......................40

Figure 3-4 Lognormal Distribution for VOT Based on SHRP 2 C04 Project

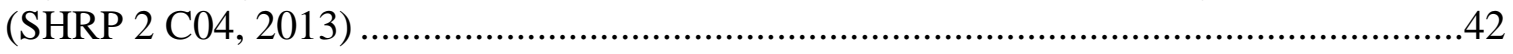

Figure 4-1 Exploration Network, Extracted Subarea from the SERPM Model ................55

Figure 4-2 Final Imported Network in NEXTA …................................................57

Figure 4-3 Demand Import Interface in NEXTA …....................................................58

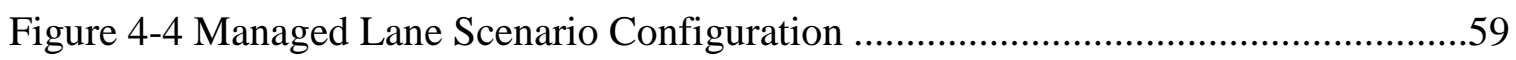

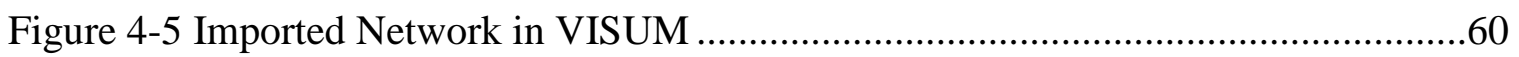

Figure 4-6 Imported Demand Matrix in VISUM...................................................60

Figure 4-7 Comparison of Different TFMs on Bottleneck Location Speed, NW $79^{\text {th }}$ St 63

Figure 4-8 Comparison of Different TFMs on Bottleneck Location Speed, NW $103{ }^{\text {rd }}$ St63

Figure 4-9 Speed Contours for the Study Corridor Utilizing Different TFMs .................67

Figure 4-10 Schematic of Sensor Locations for the ODME Process..............................69

Figure 4-11 Comparison of Observed vs. Simulated Link Volume Produced by Cube

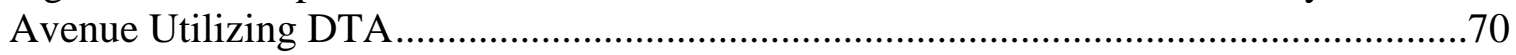

Figure 4-12 Comparison of Simulated vs. Observed Link Volumes after Running ODME in Cube 
Figure 4-13 Comparison of Observed vs. Simulated Volume Produced by VISUM Utilizing DTA

Figure 4-14 Comparison of Simulated Link Volumes vs. Observed Link Volume after Running ODME in VISUM.

Figure 4-15 Comparison of Observed Link Volumes vs. Simulated Link Volume Produced by DTALite Utilizing DTA

Figure 4-16 Comparison of Simulated Link Volumes vs. Observed Link Volume after

Running ODME in DTALite .74

Figure 4-17 VOT Distribution in DTALite (DTALite User Manual, 2012) ....................77

Figure 4-18 Comparison of Diverted Volume to ML for Different VOTs .79

Figure 4-19 Comparison of Diverted Volume to ML without VOR Utilizing Different Tools

Figure 4-20 Comparison of Diverted Volume to ML with VOR Utilizing Different Tools

Figure 4-21 Comparison of Toll-Density Curves for Old and New Toll Policies for I-95 Northbound Utilizing Different Tools ....

Figure 4-22 Comparison of Diverted Volume to ML Using Old and New Toll Policies for I-95 Northbound Utilizing Different Tools

Figure 4-23 Impacts of CACC Proportion on Lane Capacity (Shladover et al., 2012) .....88

Figure 4-24 Variation of Percentage of ML Share with Respect to CACC Market

Penetration Using STA

Figure 4-25 Variation of Percentage of ML Share with Respect to CACC Market Penetration Using DTA.

Figure 4-26 Variation of Speed at Bottleneck Location with Respect to CACC Market Penetration Using STA

Figure 4-27 Variation of Speed at Bottleneck Location with Respect to CACC Market

Penetration Using DTA. 


\section{ABBREVIATIONS AND ACRONYMS}

\begin{tabular}{|c|c|}
\hline $\mathrm{ACC}$ & Adaptive Cruise Control \\
\hline AV & Autonomous Vehicle \\
\hline CACC & Cooperative Adaptive Cruise Control \\
\hline $\mathrm{CV}$ & Connected Vehicle \\
\hline DTA & Dynamic Traffic Assignment \\
\hline DTALite & Dynamic Traffic Assignment light version \\
\hline ELToD & Express Lanes Time of Day \\
\hline FHWA & Federal Highway Administration \\
\hline FITSEVAL & Florida Intelligent Transportation System Evaluation Tool \\
\hline GPL & General Purpose Lanes \\
\hline ISSTA & Integrated Support System for Transportation Analysis \\
\hline MAE & Mean Absolute Error \\
\hline MAPE & Mean Absolute Percentage Error \\
\hline ML & Managed Lane \\
\hline MRM & Multi-Resolution Modeling \\
\hline NEXTA & Network EXploration for Transportation Analysis \\
\hline ODME & Origin-Destination Matrix Estimation \\
\hline RMSE & Root-Mean-Square Error \\
\hline SERPM & South East Florida Regional Planning Model \\
\hline STA & Static Traffic Assignment \\
\hline
\end{tabular}


TFM Traffic Flow Model

VOR Value of Reliability

VOT Value of Time 


\section{CHAPTER 1}

\section{INTRODUCTION}

\subsection{Background}

Modeling of transportation systems is an essential component of transportation system planning and engineering. Transportation modeling tools are generally categorized as sketch planning, macroscopic, mesoscopic, or microscopic based on the resolution in which traffic flows are simulated. Some of these models have static or dynamic traffic assignment to predict route selections by travelers. The traffic models of regional demand models are considered as macroscopic models. The demand forecasting models generally utilize a simple macroscopic traffic flow relationships to assess network performance during the demand modeling steps. Microscopic models are utilized to model smaller networks and are able to model individual vehicle interactions at small time intervals. Mesoscopic models have more detailed traffic representation than macroscopic models, but lower representation than microscopic models. Mesoscopic models allow the modeling of sub-networks such as small to medium regional networks. Mesoscopic simulation models generate and track individual vehicles. However these models describe the relationship between congestion and travel time through macroscopic traffic flow parameters such as volume, density, and speed.

Existing transportation tools are different in their applications and data requirements. Less detailed tool types can be used for large networks, while more detailed tool types are appropriate for smaller networks. All tool types are useful for 
transportation analysis and a combination of these tools may be proper for different applications depending on network size and the level of analysis.

Depending on the level of details, each tool type can be applied in the modeling process. An integrated approach of the different modeling levels can produce a powerful modeling environment. However, selecting, integrating, and interfacing different tools are the big challenges in transportation modeling. Therefore, the best methods for integrating and the benefits of combining these tools need to be clearly documented. Although this has been discussed in recent years, clear methods and assessments still need to be done.

Advanced modeling tools and methods are specially needed for the analyses of congested conditions and advanced Intelligent Transportation Systems (ITS) applications such as managed lanes (ML), smart work zones, incident management, freight corridors, and integrated corridor management. Depending on the level of the analyses and the specific problem under consideration, a number of tools have been used to assess these strategies including tools that can be classified as sketch planning, Dynamic Traffic Assignment (DTA), macroscopic simulation models, mesoscopic simulation models with different levels of details, microscopic simulation models, and a combination of these tools. The modeling community is increasingly faced with challenges when selecting and combining these tools for the modeling of congested conditions and advanced strategies (Hadi et al., 2013).

A Multi-Resolution Modeling (MRM) approach is proposed in this study for combining modeling tools with different traffic analysis resolutions of ML and associated advanced strategies. The resolution is the level of detail and precision used in the 
representation of the transportation network in a model or simulation. There are two definitions of MRM given by Davis and Hilestad (1998):

1. Building an integrated model involves different levels of simulation

2. Using a combination of the consistent models of the same project with different levels of modeling

An example of a simple partial MRM is to use demand forecasting models to provide initial demand estimates to mesoscopic or microscopic modeling tools. A full MRM utilizes a mesoscopic simulation-based DTA models for a large sub-area using trip demands from the demand models, and produce input data. Then, the microscopic models can be used to provide detailed analyses of selected sub-areas, corridors, or facilities. The MRM addresses issues that are beyond the capabilities of macroscopic, mesoscopic, and microscopic models. Examples of the MRM are shown in Figure 1-1 (FHWA, 2013a).

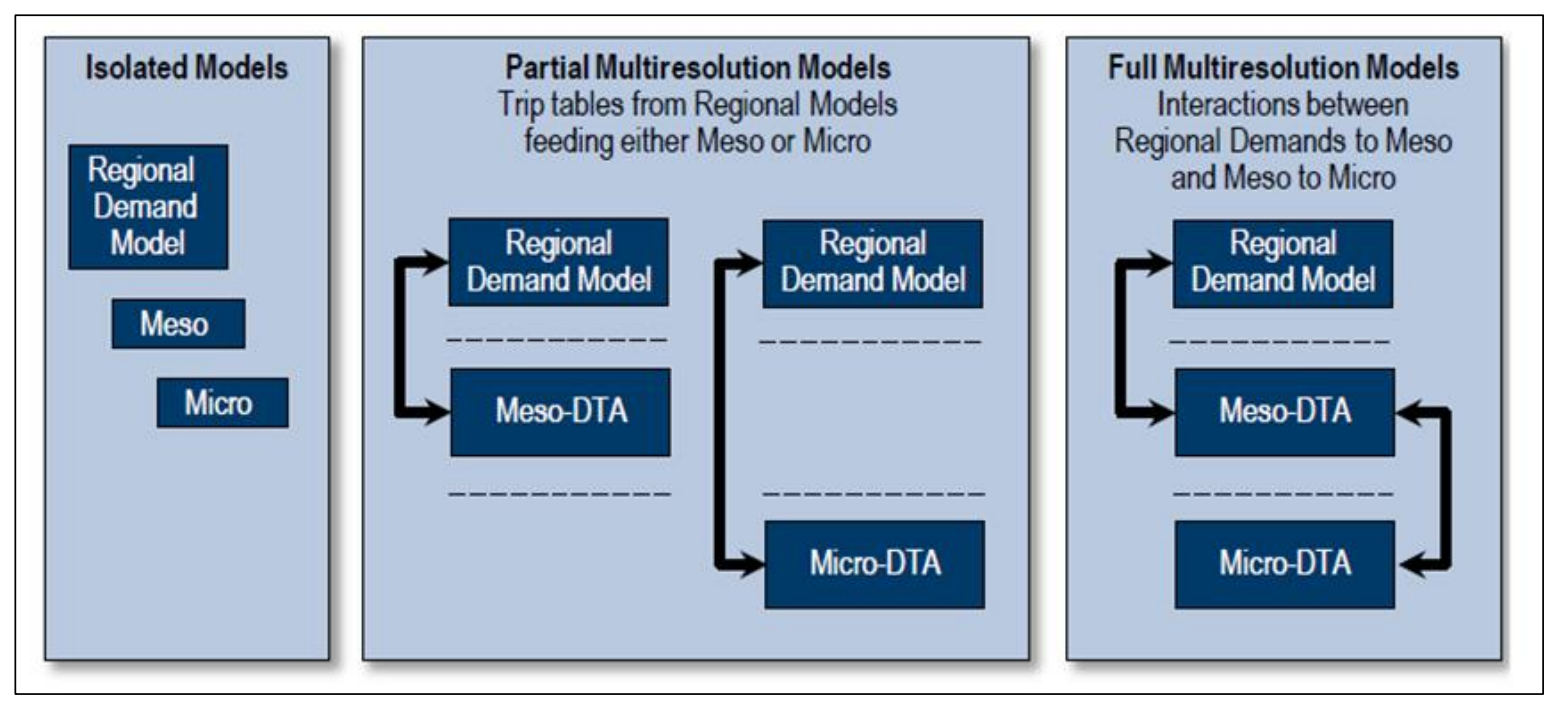

Figure 1-1 Examples of MRM Structure (FHWA, 2013a)

A typical application of the MRM approach is used in networks in which the initial demand matrices are estimated based on the approved regional demand forecasting process and then wide area diversion and bottleneck impacts are modeled using 
mesoscopic-based DTA models, followed by detailed analysis of traffic operations using microscopic models.

The Federal Highway Administration (FHWA) traffic analysis toolbox documents have provided guidance regarding the use of traffic analysis tools including simulation and DTA modeling tools, as can be found at http://ops.fhwa.dot.gov/trafficanalysistools. However, it is necessary to build on the existing state of practice and research and development efforts to establish a comprehensive framework for multi-resolution analyses to support the modeling processes. It is clear that additional tools and methods will have to be built and developed to support MRM (FHWA, 2012a).

Addressing the abovementioned needs is critical to modeling advanced strategies such as managed lane, ramp metering, smart work zone, and freight corridor management. This research investigates the use of MRM for managed lane strategy analysis with consideration of advanced strategies such as autonomous and connected vehicles.

\subsection{Problem Statement}

Modeling of transportation systems is an important component of transportation system engineering and planning. Transportation modeling tools are generally categorized as sketch planning, macroscopic, mesoscopic, or microscopic based on the resolution of traffic flows. Existing transportation modeling simulation types are different in their implementations and data requirements. Microscopic traffic simulation provides a detailed performance of traffic congestion as well as the interaction between individual vehicles. However, modeling a large network of microscopic simulation models can be 
costly and time-consuming. Previous experience shows that the development of newly integrated traffic simulation models could be more useful by linking them to regional travel demand, mesoscopic DTA-based, and microscopic models. (Rousseau et al., 2008).

Modeling tools used in transportation analyses are not integrated well enough to provide a complete and accurate assessment of the transportation systems. The need for model integration is increasingly recognized considering the needs to support the decision-making process and the tools supporting. Agencies need to evaluate many strategies by using tools that are sensitive to supply and demand calibration. In turn, this requires the use and integration of analysis tools across multiple resolutions (macroscopic, mesoscopic, and microscopic). Other data analysis and modeling support tools are also necessary for an effective modeling process (FHWA, 2013a).

One of the potential applications of MRM is the modeling of ML strategies. The utilization of advanced simulation methods is required accurately to assess the impacts of the ML and the associated operation strategies. Also, agencies require knowing the amount of the diverted volumes to a managed lane, which affected the revenue and congestion on both the ML and the General Purpose Lanes (GPL). They also need to know how the traveler will respond to changes in toll policies, such as changing the toll schedule or vehicle occupancy restriction. These questions can be answered by utilizing advanced modeling combined with network and demand calibration, and validation methods (Hadi et al., 2013).

Simulation-based DTA tools at a different level of analysis can be utilized to simulate the dynamics of traffic flow, particularly under congested conditions with consideration of queue spillback effects on traffic patterns after each assignment iteration. 
However, the data required for calibrating DTA models and the demand calibration effort are main concerns of modelers and planners when considering the use of such these dynamic traffic models (Hadi et al., 2012a). DTA models can be designed for several types of pricing strategies such as fixed, time-of-day, and congestion pricing. DTA models are able to consider a route-choice behavior problem in managed lane utilization. DTA are able to apply the concept of a generalized cost approach in modeling the selection of managed lanes by traveler. The generalized cost function is a utility function of travel distance, travel times, travel time reliability, and lane monetary cost (FHWA, 2012a).

Pricing, vehicle restriction, or access control can be used to improve system performance of the ML. In this regard, operating ML with preferential treatments of vehicles with advanced technologies such as Autonomous Vehicles (AV) or Connected Vehicles $(\mathrm{CV})$ could be beneficial since the performance of these vehicles are expected to improve the mobility and safety of ML. There will be also a time when the designation of special lanes for these vehicles will be advantageous to commuters. Special lanes for vehicle eligibility are established through High Occupancy Vehicle (HOV) lanes, High Occupancy Toll (HOT) lanes, truck lanes, and so on. It should be noted, however, that the managed lanes with vehicle eligibility restrictions present enforcement challenges. In addition, vehicle restriction and eligibility must be implemented at the appropriate time, considering the proportion of the vehicle types in traffic. The market penetration of advanced vehicle technology will also be driven by the purchase rates of these advanced vehicles and the replacement rates of conventional vehicles (FHWA, 2012b). To assess the interactions between advance vehicle technology and manually driven vehicles, it is 
necessary to use advanced models of each that have sufficient details to capture their interactions. A MRM approach that combines macroscopic, mesoscopic, and microscopic simulation modeling with DTA is proposed in this research.

\subsection{Research Goal and Objectives}

The goal of this dissertation is to develop and assess methods and tools using MRM in the evaluation of ML strategies. This research assesses the effectiveness of MRM methods and tools in the modeling of ML in different levels of analysis with the consideration of advanced vehicle technologies. The specific objectives of this study are including:

1. Develop and test criteria to select modeling tools for ML strategies.

2. Investigate the ability of existing tools with different levels of modeling to meet the ML modeling criteria.

3. Develop methods for use in support of agency modeling of ML utilizing MRM.

4. Apply and assess the recommended MRM methods in the modeling of ML.

5. Apply different pricing approaches in the modeling of ML.

6. Assess the use of MRM in the modeling of Adaptive Cruise Control (ACC) and Cooperative Adaptive Cruise Control (CACC) as examples of autonomous and connected vehicle strategies, respectively.

\subsection{Research Contributions}

This study aims to explore the effectiveness of different levels of modeling when used separately and in combinations. This study demonstrates how MRM can be used to 
answer questions related to the effectiveness of ML strategies with different incentives (preferential treatments), pricing strategies, and access restrictions to improve system performance. In particular, this dissertation shows how tools with different resolution levels can be used to model diversion due to changing toll schedules and the benefit of providing access and intensives for AVs and CVs.

A number of questions related to MRM are answered in this dissertation. A question that has been asked is what the value of going to more detailed resolutions such as utilizing dynamic traffic assignment combined with mesoscopic and possibly microscopic simulation for ML modeling. This study demonstrates the ability of different tools in modeling the operations of ML and GPL. Transportation agencies have also used models with supply and demand parameters that are not well calibrated based on detailed real-world data. This dissertation answers the question of the value of resources in better identification of modeling parameters.

This dissertation then answers a third question, which is tools with different resolutions can predict shifts to ML volume to changes in ML policies such as changes in toll schedule, that has not been adequately answered in previous studies. Answering this question is critical for the decision to select tools with specific resolutions for the agencies.

The impacts of using different parameters such as Value of Time (VOT), Value of Reliability (VOR), and toll in the assignment objective and utilizing fixed vs. stochastic VOT in the modeling results are also investigated. A unique method for increasing reliability in the generalized cost function is developed in this study that provides an effective and easy to use the method for including reliability in the route choice behavior. 
Finally, this study demonstrates how to use the MRM to model AVs and CVs on ML modeling. This modeling allows the determination of when and how it will be advantageous to provide preferential treatments to vehicles to use MLs.

\subsection{Dissertation Organization}

This section summarizes the organization of this document by presenting an overview of the chapters in this document.

Chapter 2 presents a review and assessment of past research related to the objectives and tasks of this study. First, a review is presented of existing simulation and DTA tools with emphasis on previous experience with MRM. Second, a review is presented for ML modeling approaches at different levels of details ranging from sketch

planning tools to microscopic simulation modeling. Chapter 2 finally reviews existing literature on the effects of $\mathrm{AVs}$ and $\mathrm{CVs}$ on traffic operations and illustrates the potential positive impacts on traffic mobility.

Chapter 3 presents the methodology and tasks that have been carried out through this study. This chapter explains the task sequence and the link between different tasks. The tasks of this study are including:

- $\quad$ Data sources and pre-processing

- Tool exploration and selection

- Model input/output conversion

- Supply calibration

- $\quad$ Trip demand estimation

- Application of MRM to ML 
- $\quad$ Assessment of the mobility impacts of $\mathrm{AVs} / \mathrm{CVs}$ in $\mathrm{ML}$

Chapter 4 presents the results of using MRM to model ML for a case study utilizing the methodology explained in Chapter 3.

Chapter 5 summarizes the findings of this research in the MRM framework in the context of managed lane modeling with consideration of $\mathrm{AVs} / \mathrm{CVs}$. 


\section{CHAPTER 2}

\section{LITERATURE REVIEW}

This chapter presents a review and assessment of past researches related to the objectives and tasks of this study. First, a review of existing simulation and DTA tools with focus on MRM is presented. Second, a review is presented for ML modeling approaches at different levels of details ranging from sketch planning tools to microscopic simulation modeling. Eventually, existing literature on the effects of advanced autonomous and connected vehicle technologies on traffic operations, illustrating the potential positive impacts on traffic mobility is reviewed in this chapter.

\subsection{Multi-Resolution Modeling (MRM)}

Analysts need to use a combination of tools with different resolutions, functionalities, and capabilities to take a full advantage of advances in traffic modeling. However, one main problem that arises when using a combination of different modeling tools is the difficulty of using the interfacing and integrating processes, as well as using data from various data sources and resolutions (Holyoak and Branko, 2009). The interfaces between tools have been in one direction, from macroscopic level to the microscopic level, in the most previous applications of MRM. However, two-way interfacing is also possible, and has not been adequately considered in previous transportation modeling levels (Duthie et al., 2012). 
The modeling of advanced vehicle technologies in this dissertation can actually be considered as a full MRM. The research on MRM is in its initial stages and there is still a lot to be learned regarding this type of analyses.

\subsubsection{Review of Simulation and Dynamic Traffic Assignment (DTA) Tools}

DTA is becoming an increasing acceptance in the transportation community. DTA models provide a more realistic traffic flow and driver response modeling patterns compared to static models used in traditional demand forecasting models by accounting for changing traffic conditions in small time intervals (15-30 minutes is usually used). More details about DTA can be found in Hadi et al. (2013), Hadi et al. (2012a), Hadi et al. (2012b), Chiu et al.(2011), and FHWA (2012a).

One of the main benefits of using DTA to model traffic networks is that the outputs of the model describe the time-dependent network states. Outputs from the model include time-dependent system level, and link level performance statistics.

Based on the discussion in Hadi et al. (2012b), DTA models are able to capture many realities in the transportation network that static assignment cannot capture, including vehicle trajectories, traffic performance, and driver routing decisions in response to congested conditions and advanced management and pricing decisions. A comprehensive review of DTA-based tools and their approaches was conducted by Hadi et al. (2012a) in IITS research lab at Florida International University (FIU). The summary of the review is presented in Appendix 1.

The simulation-based DTA tools described in Appendix 1 vary in the level of details from macroscopic to mesoscopic to microscopic. Tools with different levels of 
resolution are suitable for different applications. However, combining these tools in a single application can provide capabilities and functionalities that are not possible with the use of one type of model as described in the next section.

\subsubsection{Previous Experiences of MRM}

MRM refers to a modeling framework that combines microscopic, mesoscopic, and macroscopic representations of traffic flow. There is a consensus that such framework can be a useful tool to better assess traffic operations and advanced strategy impacts.

The Federal Highway Administration's (FHWA) Office of Operations classified the structures as partial and full MRM (FHWA, 2012a), as shown in Figure 2-1.

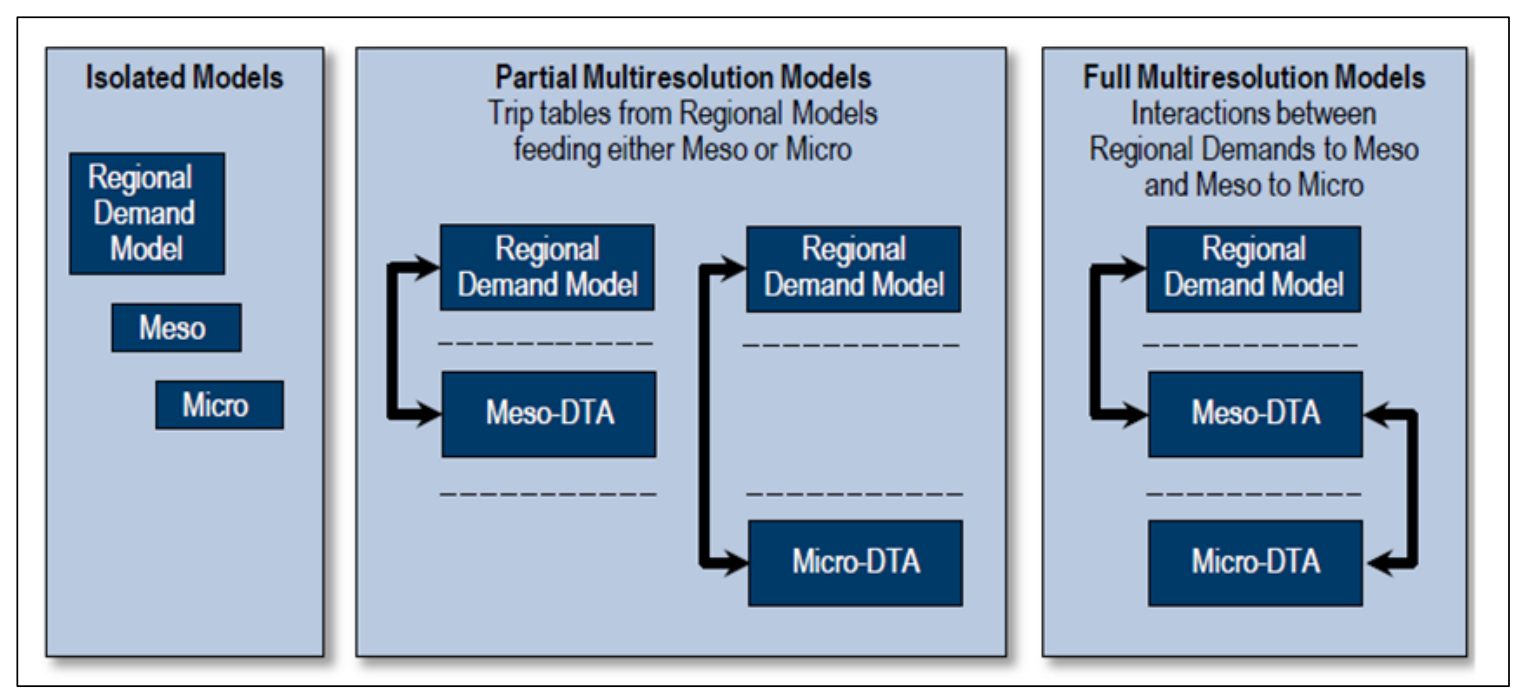

Figure 2-1 Example of Modeling Structures (FHWA, 2012a)

Sbayti and Roden (2010) compared the use of a partial MRM (macroscopic model to microscopic model structure) versus a full MRM (macroscopic to mesoscopic to microscopic model structure). In the partial MRM, a sub-area from the demand forecasting model was converted to run in a microscopic simulation tool. When using a 
full MRM approach, the outputs from the macroscopic model were fed into a mesoscopic DTA model, which produces time-dependent flows that are capacity constraints, and are used as inputs to microscopic models. They also identified the common challenges to integrate the regional demand models and network simulation tools including network and demand calibration, network input and output conversion, and hardware processing to support the integration of different levels of modeling.

In recent years, a few studies have used MRM in practices. A combination of the DynasT mesoscopic tool and VISSIM microscopic tool was used by Shelton and Chiu (2009). In their study, a sub-area was cut from a large regional DynusT network. To conduct this process, a conversion tool was developed to read files from DynasT inputs and outputs and generated the corresponding network and demands in the format required by VISSIM. Figure 2-2 illustrates the modeling framework that was used by Shelton and Chiu (2009).

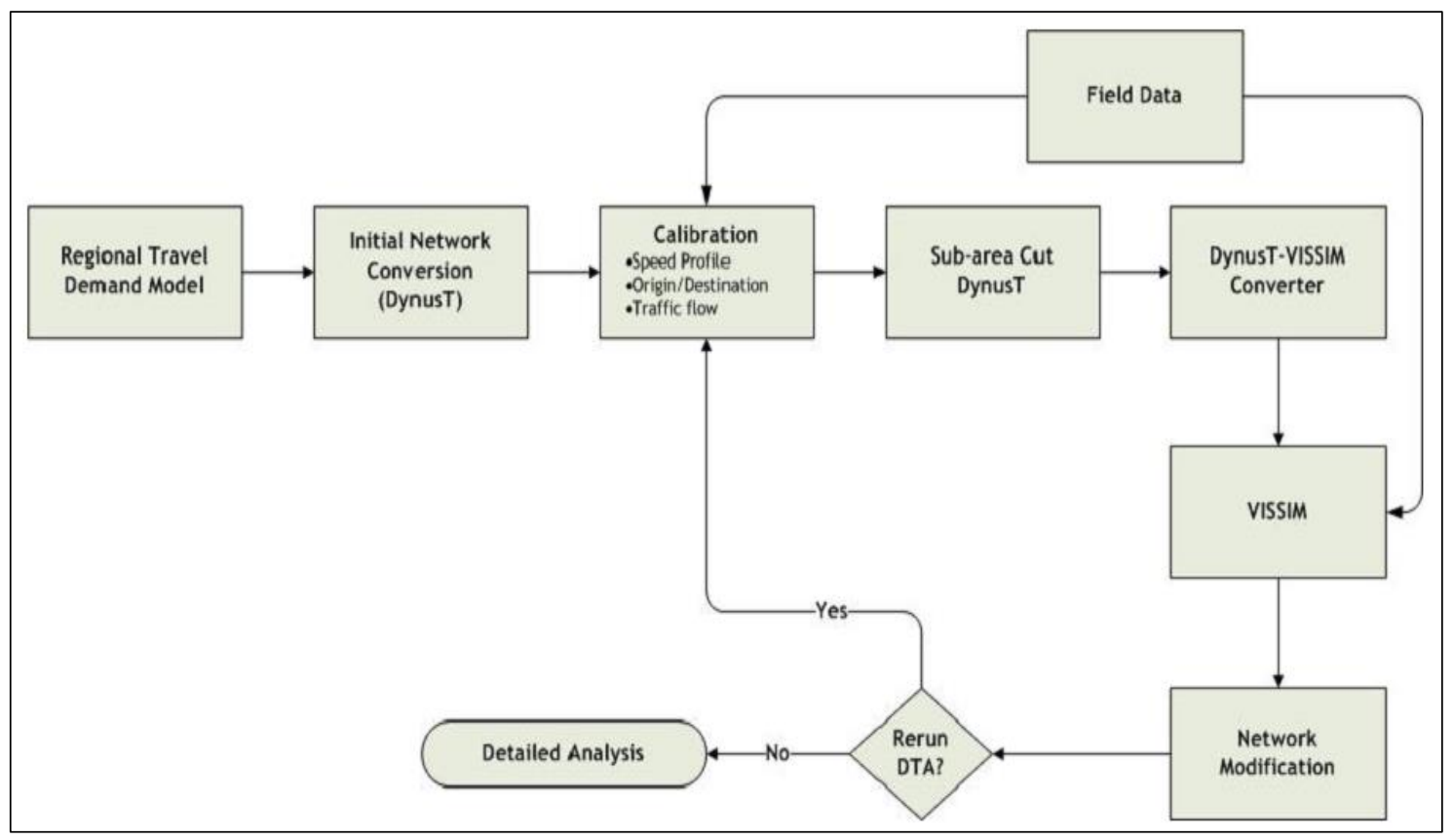

Figure 2-2 Modeling Framework for Mesoscopic-Microscopic Integration (Shelton and Chiu, 2009) 
Martin al. (2011) developed a partial MRM framework that involved the macro and microscopic simulation models. The travel demand forecasting model was implemented in VISUM simulation tool. A cut subarea from the VISUM macroscopic model was fed to the VISSIM microscopic simulation tool. Duthie et al. (2012) also used a combination of TransCAD (macroscopic demand forecasting model), VISTA (mesoscopic simulation-based DTA model), and VISSIM (microscopic simulation-based DTA model).

In summary, it can be concluded that MRM has recently been referenced as an approach to improve the assessment of the impacts of time-varying traffic demand that are capacity constrained and the associated vehicle routings that can be used as inputs to microscopic simulation model.

\subsection{Managed Lane (ML) Modeling Structure}

ML strategies are innovative ITS strategies to address congestion problems. These strategies involve operating lanes adjacent to the GPL of a freeway facility and providing congestion-free trips to eligible users. A Combination of access control, pricing, and vehicle eligibility defines the different types of ML. ML policies are adjusted to local traffic conditions. The toll values are different based on the time-of-day and the level of congestion. In an advanced toll policy, different toll values are applied to different user groups utilizing dynamic congestion pricing schemes (Hadi et al., 2013).

The key feature that distinguishes ML from traditional capacity improvements is the operational flexibility to actively respond to the current situation, and continuously keep the facility in optimal conditions. The criteria to select the operation parameters of 
ML can include one or more of the following: preserving a certain level of service in the ML, maximizing revenue, supporting environmentally-friendly vehicles, improving trip reliability, improving safety, and encouraging the use of public transit (FHWA, 2008c). Although the objective of the strategies utilized in existing managed lane applications is mainly to maintain an acceptable level of service of the priced lanes, studies show that travelers in general purpose lanes also benefit from managed lane deployments (Safirova et al., 2013; and Janson \& Levinson, 2013).

Successful implementation of ML strategies requires using advanced modeling methods to allow better assessment of the impacts of changes in traffic conditions and the impact of operation strategies. Simulation-based DTA tools are suitable candidates for use in ML modeling. These tools utilize simulation to capture the dynamics of traffic flow in congested conditions (Shabanian, 2014).

A variety of modeling approaches have been proposed to assess managed lane implementations. These approaches range from high-level sketch planning tools to microlevel modeling of individuals' behaviors and traffic operations (DeCorla-Souza and Whitehead, 2003; Li and Govind, 2003; Murray et al., 2001; and He et al., 2000).

\subsubsection{Sketch Planning and Demand Model-Based Tools}

The Federal Highway Administration (FHWA) developed an open-source sketch planning tool (POET-ML) to perform a quick evaluation of ML performances and pricing policies. The inputs required to use this spreadsheet include eligibility policies such as occupancy restrictions; physical characteristics such as the lengths and the number of the lanes, median types, and buffer types; and demand information such as the peak hour 
volumes on ML and GPL facilities. The user can change the current policy according to the results produced by the tool. The outputs from the tool include the potential impacts on travel demands, revenues, mobility, and the environment (FHWA, 2008a).

Another spreadsheet-based application, developed by the University of Texas at Austin is the Project Evaluation Toolkit (PET). PET includes a trip demand estimation module for time-of-day modeling and route choice behavior with different demand types. MLs may be directly implemented in PET by adjusting tolling rates by vehicle type and time of day. Vehicles types can be excluded from using the HOV and ML lanes by setting the tolls extremely high (Kockelman et al., 2012).

The Florida ITS Evaluation (FITSEVAL) is another sketch planning tool developed for the Florida Department of Transportation (FDOT) by Hadi et al. (2008) at Florida International University in Miami, Florida to evaluate and assess ITS advanced strategies through the Florida Standard Urban Transportation Model Structure (FSUTMS) framework. This tool evaluates the effects of different ITS applications including ML, on network performance measures such as Vehicle Mile Traveled (VMT) and Vehicle Hour Traveled (VHT), crash statistics, emissions, average speed, and fuel consumption. The ML module in FITSEVAL utilizes the static assignment of the Cube software.

The FHWA developed an interactive spreadsheet sketch planning tool, referred to as the Tool for Rush-Hour User Charge Evaluation (TRUCE) to quantify the impacts of congestion pricing on urban highways. In its current form, the model considers scenarios for congestion pricing on the network. The pricing strategy is changed based on the travel time savings (FHWA, 2008b). 
The Florida Department of Transportation (FDOT) in 2013 developed a standard approach for managed lane demand forecasting applications in the FSUTMS as a project with different phases. Phase I focused on the use of static assignment in developing route choice in managed lane modeling and analysis. The developed model was used to determine the proportion of drivers willing to use ML, given the charged tolls and the difference in performance between ML and GPL lanes, based on a static assignment procedure combined with a willingness-to-pay curve (Ruegg et al., 2013). In Phase II, the choice between GPL and ML was formulated by a logit model in the mode choice step of the traditional four-step demand forecasting procedure (Parsons Brinckerhoff, 2013).

The Florida's Turnpike Enterprise (FTE) in 2012 focused on estimating the express lanes traffic operations as a function of variable toll rates. FTE developed a tool which is referred to as the Express Lanes Time of Day (ELToD). ELToD utilizes a dynamic pricing policy, VOT, and VOR in the evaluation. An important input to ELToD is the O-D matrices which are output from the travel demand models. ELToD assumes the daily traffic and hourly distribution constant and estimates the diversion that will occur between the general purpose lanes and express lanes given these volumes. This can be done by solving the supply/demand equilibrium problem considering both the toll level and travel times for each analysis time interval (FTE, 2012).

\subsubsection{Data Analytical Tool}

The ITS Data Capture and Performance Management (ITSDCAP) is real-world data-based evaluation and analysis tool that was developed for the FDOT by Hadi et al. (2012c) at Florida International University. One of the functionalities of this tool is to 
evaluate and analysis ITS implementation. Procedures were developed in the ITSDCAP tool to evaluate advance ITS strategies such as managed lanes, ramp metering, incident management, and smart work zone. The benefits of managed lanes should be evaluated for both planning and operational purposes. The impacts of MLs are considered in this tool by the performance measures including travel time, travel time reliability, maximum throughput, and toll revenue.

\subsubsection{Modeling Input Parameters}

Two essential components of the managed lane choice are the Value of Time (VOT) and Value of Reliability (VOR). VOT is the measure of a driver's willingness-topay for travel time savings. VOR is a component that affects a traveler's decision to choose the ML, and is expected to be a function of travel distance, income, and car occupancy.

The FDOT evaluated the modeling of ML in Southeast Florida Regional Planning Model (SERPM) model (FDOT, 2013). The calibration of the ML model within the SERPM framework identified the use of a VOT of $\$ 11.75 /$ hour, and a range of $\$ 0.00$ to $\$ 2.99$ for VOR. The VOR in this estimation seems to be low. This value is based on stated and revealed preference surveys from fall 2011 (Resource Systems Group, 2012).

Calibrating models based on state and revealed preference surveys for the Florida Turnpike's tolling model resulted in a VOT ranging from $\$ 3 /$ hour to $\$ 13.50 /$ hour, based on trip purpose and income level (Dehghani et al., 2003). A survey conducted by the Florida's Turnpike Enterprise (FTE) in 2005 showed that $91 \%$ of the responders perceived the benefit of paying the toll in terms of service, safety, and convenience (FTE, 
2005). Nava et al. (2013) selected a VOT of $\$ 15.50 /$ hour for SOV and HOV users and a VOT of $\$ 46.50 /$ hour for commercial trucks.

Recent findings recommended adding travel time reliability as a decision factor in the assignment process, and introducing VOR in the generalized cost function. (Cambridge Systematics. Inc, 2012). In order to obtain travel time reliability from stated and revealed preference surveys, the Resource Systems Group (2012) associated travel time reliability with travel time distribution entropy. The entropy was calculated as a function of the mean and standard deviation of the travel time distribution. The value of reliability was estimated in dollar per unit of entropy.

Minnesota was the first state to implement a dynamic pricing algorithm that updates the toll based on HOT lane density and density variability every three minutes, with a goal to keep the level of service at C (Janson and Levinson, 2013). Tolls charged to vehicles were adjusted every three minutes according to HOT lane vehicle density. In this research, dynamic pricing approach in managed lane is applied to model the managed lanes.

The Strategic Highway Research Program SHRP 2 C04 project (2013) conducted a number of policy implications in terms of VOR and VOT and found a range of VOT from $\$ 5$ to over $\$ 50$ per hour and increasing by $\$ 100$ per hour when trip demand is high. Therefore, toll values and policies had the significant impacts in the congestion condition on ML. In the SHRP 2 C04 project, a lognormal distribution is assumed for the distribution of the value of time. The SHRP 2 C04 research team also evaluated the reliability ratio (VOT/VOR for an average trip distance). They found ratios in the range 
of 0.7 to 1.5 for various model specifications based on stated preference (SP) survey (SHRP 2 C04, 2013).

Another important input parameter in ML modeling is link capacity for ML. The Highway Capacity Manual (HCM) is the primary source for estimating highway capacity for planning and operation applications. In addition, estimating capacity based on realworld data rather than the HCM method is recommended when real-world data is available, especially when there are observations that the capacity at the real-world is different from the average conditions recommended by the HCM.

A number of approaches and methods were proposed to estimate the capacity measurement for the modeling of ML. Some researches focused on the capacity estimation based on the different demands in different time intervals over different days (Dervisoglu et al., 2009; Chao et al., 2005; and Jia et al., 2010). Other researches were conducted to approximate the capacity by fitting data into fundamental diagrams such as speed-flow and flow-occupancy models (Bassan and Polus, 2010; Rakha and Mazen, 2010; and Wang et al., 2012).

\subsubsection{Dynamic Traffic Assignment (DTA) Implementation in ML Modeling}

The impacts of advanced strategies such as ML are particularly significant when

the facility is operating near its capacity. Applying these strategies is time-dependent and highly sensitive to changes in traffic flow performance. Therefore, these applications require more advanced and detailed modeling frameworks, compared to the approaches used in traditional demand forecasting. The use of simulation-based DTA has been proposed as a modeling method to provide more accurate and reliable analyses of ML. To 
better understand the difference between STA and DTA, it is necessary to first understand the main components of traffic assignment procedures that run sequentially and iteratively seeking a convergence. These three main components are including network loading, shortest path identification, and trip demand assignment to the identified alternative path (Shabanian, 2014 and Hadi et al., 2013).

The I-95 managed lane facility in Miami, Florida, was evaluated in a study conducted by Shabanian (2014) using two different approaches including managed lane costs in the objective function, which is an approach traditionally applied in toll modeling, and utilizing a willingness-to-pay curve in conjunction with the DTA. The ML modeling in the abovementioned study contains a toll diversion process, as well as a congestion-based (dynamic) tolling selection process, so that it estimates the toll trips and the toll costs for each time segment in the managed lanes.

The application of a simulation-based DTA to the evaluation and operation of ML lanes was investigated by Abdelghany et al. (2000). The Dynasmart is able to model dynamic route choice behavior in ML modeling. A special purpose k-shortest path algorithm was included in Dynasmart tool. Dynasmart was able to represent several operating characteristics of the ML including lane utilization in terms of adding a new lane to the facility, physical separation of the ML, ML pricing approaches, and preferential treatments.

Kerns and Paterson (2011) developed a dynamic toll algorithm to the toll prices at based on traffic density. They used the micro simulation-based DTA in the TransModeler tool to enhance the development and implementation of a dynamic toll algorithm. 


\subsection{Mobility Impacts of Advanced Vehicle Technology}

Advanced vehicle technologies such as $\mathrm{AVs} / \mathrm{CVs}$ are expected to change the traffic flow with consideration of car-following, lane-changing, and gap acceptance modeling of individual vehicles. The is still a need to assess the impacts of these advanced vehicle technologies if these vehicles systems are implemented in combination with other ITS advanced strategies like ramp metering, managed lanes, and integrated corridor management, their effects need to be well known to justify the additional investment in these technologies.

In these coming years, the market penetrations of autonomous Vehicle (AV) and Connected Vehicle (CV) technologies are expected to increase significantly. Two examples of these technologies are Adaptive Cruise Control (ACC) as an example of autonomous vehicle technology and Cooperative Adaptive Cruise Control (CACC) as an example of connected vehicle technology. These technologies have the potential impact to increase traffic throughput by shorter following distances between vehicles. ACC is an automotive capability that allows a vehicle's cruise control system to adapt the vehicle's speed to the traffic network. Adding a wireless vehicle-vehicle communication to ACC system has been proposed by CACC systems. The CACC is developed to enhance the car-following feature of ACC utilizing shorter distance gaps than ACC. The idea of using CACC is not only to keep a proper following distance behind another car, but also to allow cars to cooperate by communicating with each other while in the adaptive cruise control mode. (FHWA, 2015; FHWA, 2013b; and Reich, 2013).

As mentioned earlier in this research, vehicle eligibility and preferential treatments on ML have been implemented into HOV lanes, HOT lanes, truck lanes, and 
so on. The market penetration of the ACC and CACC technology will be driven by the purchase rates of advanced vehicles and the replacement rates of vehicles that are not equipped with advanced technologies (FHWA, 2013b). To assess the interactions between $\mathrm{ACC}$ or $\mathrm{CACC}$ vehicle technologies and conventionally driven vehicles, it is necessary to use microscopic models that can capture the interactions between these different vehicle types (Reich, 2013).

Most of the tools used in previous studies on modeling and evaluating the impacts of ACC and CACC on traffic flow and operations are based on microscopic traffic simulation (FHWA, 2015; and FHWA, 2013b). In addition, these studies have not addressed the impacts of these technologies combined with ML strategies on ML utilization and system performance. However, there is a need to assess their interactions to ensure the best performance possible with their use as advancements are made in both connected vehicles and ML strategies. This research investigates the use of models with different resolutions for this assessment. The research utilizes models with different resolutions, including a demand forecasting model based on a macroscopic traffic flow model and a mesoscopic simulation-based DTA model, combined with the results obtained from microscopic simulation modeling to investigate the interactions between ML strategy and traffic streams with a proportion of the vehicles equipped with CACC vehicle technologies.

Microscopic simulation has been used to evaluate traffic flow performance, when considering advanced vehicle capabilities, as well as driver behavior including carfollowing, lane-changing, and time gap settings (Bifulco et al. 2013, Ngoduy, 2013, and Tapani, 2013). Kesting et al. (2007) identified significant improvements in performance 
measures with the use of ACC. According to that study, at 10 percent market penetration, the maximum travel time delays of individual vehicle can be reduced by about 30 percent and the total delay by 50 percent at $100 \%$ pet ration rates of ACC. Van Driel and Van Arem (2008) focused on the effects of ACC vehicles in congested conditions and they found that the ACC equipped vehicles significantly reduced the maximum queue length. Elefteriadou et al. (2012) also found out that ACC vehicles are able to significantly increase the speeds in bottleneck locations at a low market penetration rate of $20 \%$.

However, ACC could produce positive results in the all evaluations. Shladover et al. (2012) applied microscopic simulation modeling to estimate the effect of different market penetrations of ACC on highway capacity based on car-following behaviors. Their results showed that conventional ACC is unlikely to produce any significant changes in highway capacity because drivers selected the gap settings similar to the gaps they choose when driving manually. Similarly, Davis (2004) found that bottlenecks can occur for ACC penetration rates of $40 \%$ or more because of longer time gap selected by the drivers. Other studies (Wang and Rajamani, 2010; Arnaout and Bowling, 2014; and Calvert et al., 2012) evaluated the importance of gap setting with respect to capacity impacts of ACC vehicles. They found that ACC systems can result in longer time-gaps between vehicles to increase safety and may adversely impact reliability.

On the other hand, recent research efforts, based on microscopic simulation, confirm that the increase in market penetration of CACC will significantly increase capacity. Shladover et al. (2012) applied microscopic simulation modeling to estimate the effect of different market penetrations of ACC and CACC on highway capacity. They used AIMSUN, which is a microscopic simulation tool because it was the only simulation 
tool, in which the NGSIM model of oversaturated freeway flow could be implemented to provide the most realistic representation of manually drivers' car-following behavior in congested conditions. The results from this research showed that conventional ACC was unlikely to produce any significant changes in the capacity of the highways due to longer gap by using the ACC system. On the other hand, the CACC was able to increase capacity with a moderate to high market penetration. With CACC drivers have more confidence in using shorter gap settings. These results showed a maximum lane capacity of about 4,000 vehicles per hour if all vehicles were equipped with CACC. The lane capacity increased approximately linearly from 2,000 to 4,000 as the percentage of CACC vehicles increased from zero to one hundred. The increase in capacity is due to CACC's capability that most drivers are willing to travel at much shorter time gaps than usual. Shladover et al. (2012) also tested a scenario to assess the effects of the different combinations of ACC and CACC market penetrations based on the time gaps chosen by drivers in field tests with the remaining vehicles manually driven.

A few researchers also looked at evaluating the effect of CACC on advanced ITS strategies such as ramp metering for use during congested conditions utilizing microscopic simulation. Scarinci et al. (2013) discussed a new ramp metering strategy called Cooperative Ramp Metering (CoopRM), which takes advantage of the presence of CACC vehicles. Compared to the network without utilizing CoopRM, a microscopic simulation of the CoopRM system showed significant improvements in the traffic performance with the reduction of congestion. These results showed that the maximum on-ramp flow that can merge into gaps created by cooperative vehicles on the main line was a function of the size of the achievable gap, the main line flow, the platoon size, and 
the CoopRM cycle length. The results confirmed the reduction in the congestion area due to a better merging maneuver that was estimated to be between $50 \%$ and $70 \%$ depending on the on-ramp flow. Also, the number of vehicles not able to find a suitable gap decreased between $60 \%$ and $80 \%$.

Pueboobpaphan et al. (2010) assessed an algorithm for on-ramp merging to assist in the merging process using microscopic simulation. The algorithm encourages smooth deceleration of the mainline vehicles upstream of the merging area in order to create gaps for ramp vehicles. They considered a merging assistant algorithm for situations that the mainline traffic is combined with manual and CACC vehicles and the ramp traffic only consists of manually driven vehicles. The results showed that the effectiveness of the merging assistant algorithm can be different based on the demand and percentage of CACC vehicles on the mainline.

The most current use of tools in the modeling and evaluating the impacts of ACC and CACC on traffic flow and operations are based on microscopic traffic simulation models. Most of the assessments that used microscopic simulation models focused on analyzing these advanced technology impacts on traffic flow without the consideration of using ACC or CACC on exclusive lanes such as MLs. However, additional researches should consider the application of these technologies as advancements are made in the areas of CACC or ACC and managed lane strategies. Regional or sub-area impacts of these advanced vehicle technologies on ML facilities needs to be studied and MRM is a powerful approach to achieve this purpose, as will be investigated in this dissertation. 


\subsection{Summary}

Analysts need to use combinations of tools with different resolutions, functionalities, and capabilities to take a full advantage of advances in traffic modeling. However, one main problem to use of combinations of different modeling tools is the difficulty in interfacing, integrating, and using information from various data sources and resolutions (Holyoak and Branko, 2009). The interfaces between tools have been in one direction, from the low level of modeling details, macroscopic level, to the high level of modeling details, microscopic level, in the most previous applications of MRM. However, two way interfacing, which is full MRM, is also possible and can be beneficial, and has not been sufficiently considered in previous transportation modeling levels (Duthie et al., 2012).

A comprehensive evaluation of simulation tools and MRM strategies has been presented in this research to assess the capability of each tool. Most existing modeling efforts of ML have used toll and travel time value with consideration of VOT. Recent efforts also showed that the use of the VOR is important to better reflect the user behavior. The studies also investigated the importance of accounting for variations of VOT and VOR by the user category and the randomness of these values within each user category.

The advancements in AVs and CVs technologies require advanced tools to allow the modeling of the operations of vehicles with these technologies on ML. The studies based on microscopic simulation showed that the applications of CVs has the potential to increase capacity. Their results can be input to macroscopic and mesoscopic-based DTA tools to provide better assessment of the impacts on route diversion. 


\section{CHAPTER 3}

\section{METHODOLOGY}

The focus of this research is on developing and testing processes to use the Multi-Resolution Modeling (MRM) for assessing and selecting ML strategies utilizing different levels of analyses including sketch planning, different levels of simulation, and DTA models.

This chapter presents an overview of the methodology and tasks implemented throughout this study. In general, the methodology follows sequential steps to achieve the objectives of this research. The first step is the data collection and pre-processing, which makes use of the data that is becoming available from multiple sources with the advancements in data collection technologies and sharing. Next, a combination of tools is selected for the modeling and analysis of this research. After the tool selection, the next step is to develop a method to automate the conversion of the input and/or output data between different levels of selected modeling tools. Then, the traffic network and demand parameters (supply and demand) are calibrated utilizing real-world measures such as traffic flow, queue length, and travel time on each link. The trip matrices are estimated for short time intervals (e.g., 15 minutes or 30 minutes) as required by DTA models. The Origin Destination Matrix Estimation (ODME) processes associated with different tools are tested for this purpose. Next, an assessment is made of the ability of different tools to predict diversions between ML and GPL under different congestion levels and toll policies. This chapter also investigates the impacts of different assignment modeling parameters including the inclusion of reliability in the generalized cost function, the use 
of different VOT and VOR values, and the use of distribution of VOT. Finally, a method is presented to assess the mobility impacts of vehicles with ACC and CACC technologies on ML and GPL, when giving preferential treatments on ML. Figure 3-1 shows the methodology steps. The remaining subsections of this document discuss the methodology in more detail.

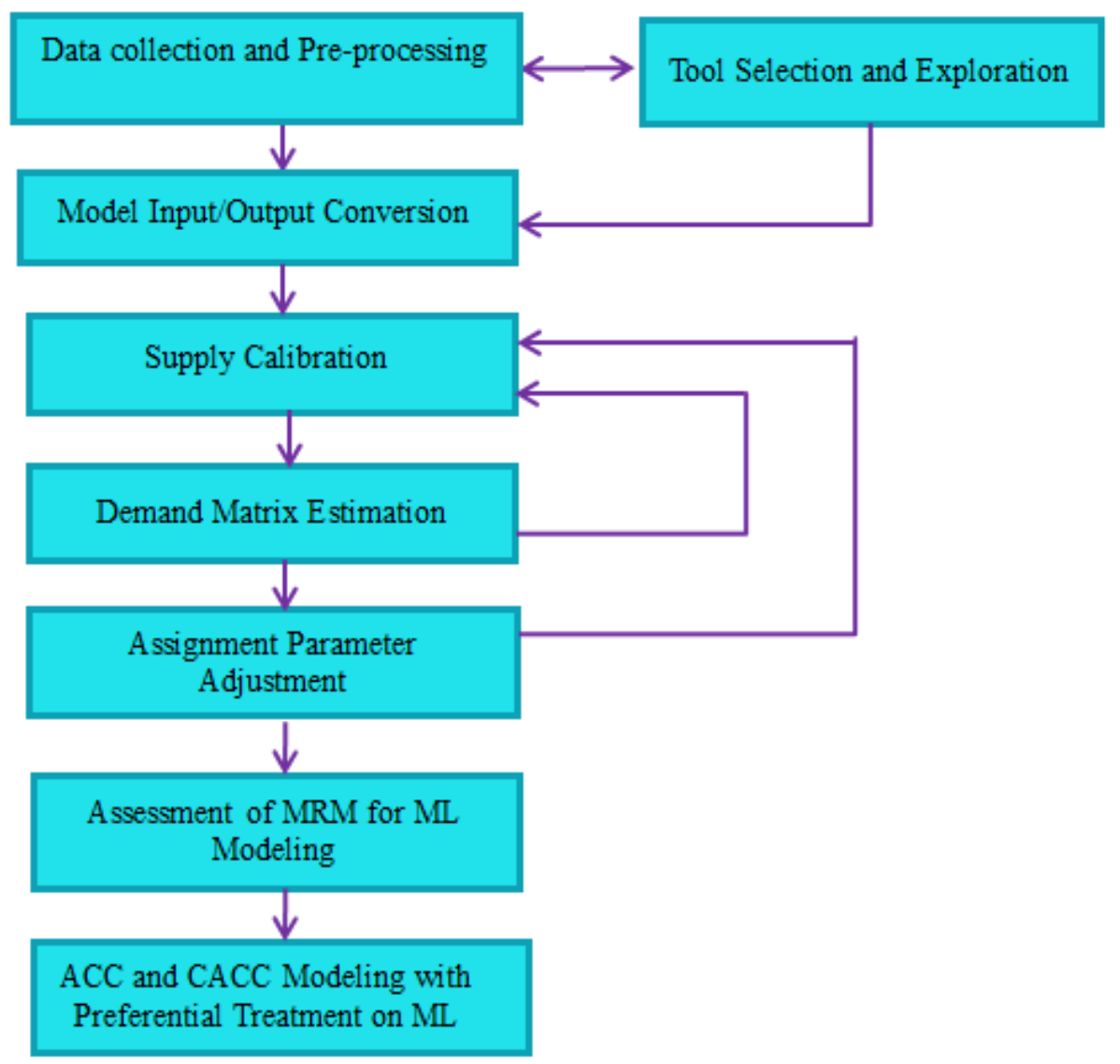

Figure 3-1 Methodology Steps

\subsection{Overview of Developed MRM Framework}

Figure 3-2 illustrates the MRM framework proposed in this project for the general analysis. As shown in this figure, the framework consists of three components: 
- Data sources and tools that allow the utilization of data from multiple sources to support modeling tasks.

- $\quad$ Supporting environment that assist modelers in developing, calibrating, and processing the results of the selected modeling tools.

- Modeling tools of different types and resolution levels that allow the estimation of various performance measures.

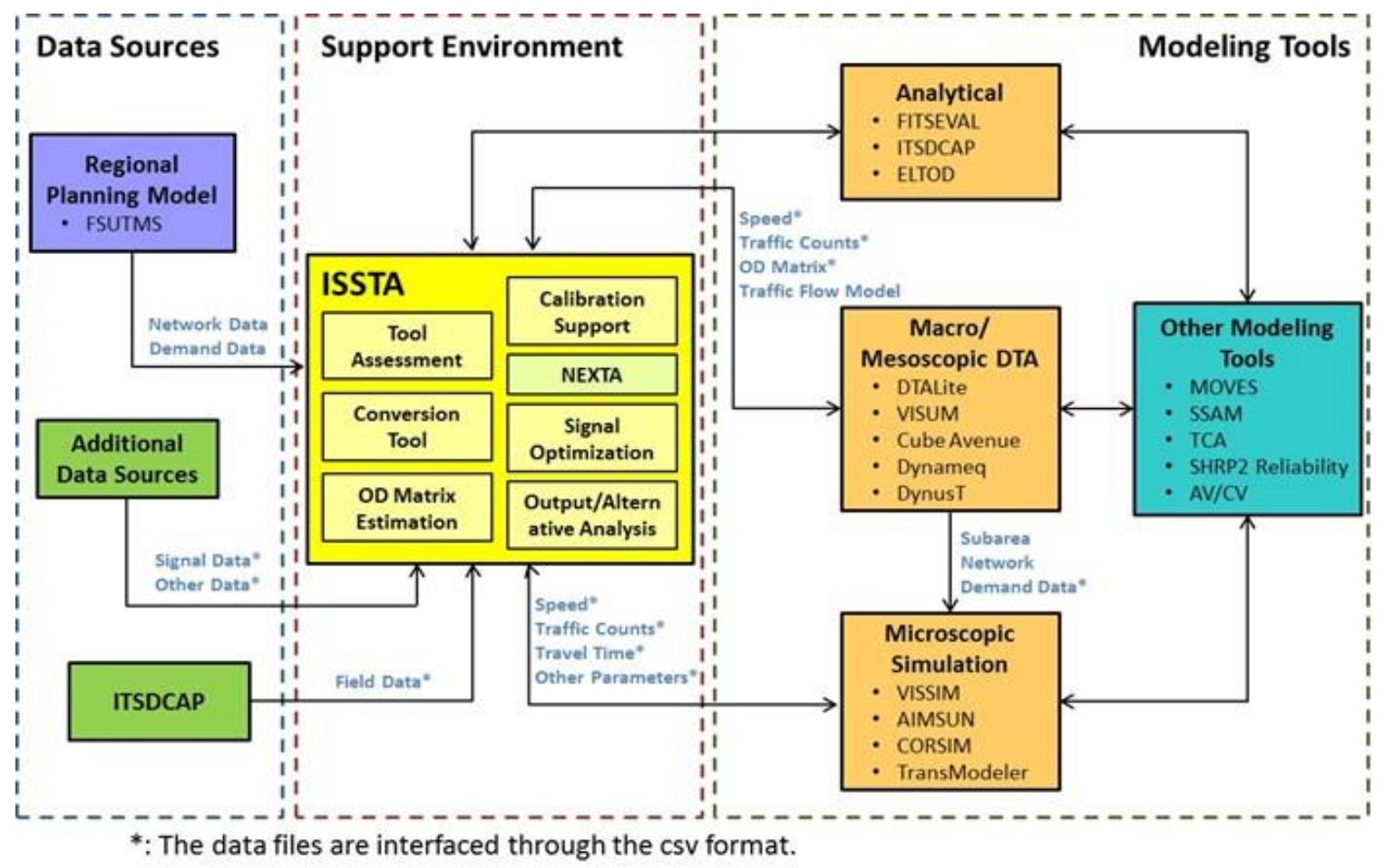

Figure 3-2 Development of General Multi-Resolution Modeling (MRM) Framework

Figure 3-2 shows a general MRM framework that could be adopted for ML modeling. The following subsections discuss the application of the framework components for the purpose of this study in the modeling of ML. 


\subsubsection{Tool Exploration and Selection}

An assessment is performed to select and use analysis tools for this research. Hadi et al. (2012a) developed a list of criteria for the assessment of simulation-based DTA tools. These criteria can be used to allow general comparisons of various modeling tools to meet the needs of the specific modeling problem. In this research, a spreadsheet is produced that lists these criteria. Additional criteria that are specific to the modeling of ML and ACC/CACC modeling are added to the list of general criteria by Hadi et al. (2012a). Some important criteria that are in the spreadsheet are mentioned below:

- Ability to constrain the demand arriving at the study link by the upstream link capacity and the downstream queuing storage.

- Ability to allow fixing paths for parts of the demands and assigning the remaining demands.

- Ability to enable a lane-by-lane simulation.

- $\quad$ Ability to model multiple types of travelers with different Willingness-ToPay (WTP) parameters.

- Ability to include various relevant factors in the generalized cost of the assignments with different coefficient values by traveler types.

- Ability to shift demands between ML and GPL using the general cost function or WTP behavioral model.

- Ability to model the impact of ACC and CACC proportion on the capacity of the link at each iteration of the assignment.

- Utilization of static versus dynamic assignment.

- $\quad$ Ability to model freeway and signalized arterial streets. 
- Ability to accommodate diversion to alternative routes.

A list of the criteria (the original and added requirements) is included in Appendix 2. It should be mentioned that this summary table is completed based on the feedback from additional reviews, vendor response, and testing of these tools. The tool selection process should be based on the project under consideration and future plans for further application.

\subsubsection{Data Sources and Pre-Processing}

Advanced modeling tools such as DTA and simulation tools require high-quality processed data to ensure that the developed model applications accurately simulate existing real-world conditions under different scenarios. The needed inputs for these simulation models with DTA capabilities, including network parameters (link length, free-flow speed, and capacity), signal timings, and dynamic demand matrices. Data sources and tools that are used to develop, validate, and calibrate the models in this research are listed below:

- Data from demand models including calibrated network data and initial demand matrices.

- Real-world data including volume, speed, occupancy, and travel time measurements from traffic detectors.

- $\quad$ ML toll schedule.

\subsubsection{Model Input/output Conversion}

As stated earlier in the literature review, an effective MRM approach to advanced strategy analysis relies on tools with different modeling levels that complimenting each 
other to deliver the required functionality. To support the use of the combination of these tools, there is a need to utilize tools that automatically convert input/output data from one tool format to another. The typical input data to DTA and simulation models include: network data, demand data, and signal control data. However, the inputs data files for different modeling tools are in different formats. Thus, the conversion tools and processes are modeled to automatically convert the inputs and sometimes the outputs from one tool to the required inputs by another tool. In addition, conversion tools and processes can also be developed to assist in converting collected real-world data to modeling tool inputs. Existing or newly developed tools by others is used in this research. The following are further descriptions of the data that need to be converted.

The modelers have to perform editing of the converted network and most likely input other parameters not available from the original source of the network. In addition, in many cases, more details have to be added to the network when using less detailed to high detailed models. As with travel demand forecasting models, one of the important inputs of advanced modeling tools is the network physical attributes. However, more detailed attributes of the network have to be added when considering from regional demand models to mesoscopic to microscopic simulation. The original networks as coded in the demand models may also have errors and inconsistencies that may not affect the regional model results but may result in errors when running more detailed models or inaccurate results. These errors and inconsistencies need to be resolved before using this network as the inputs to the more detailed models.

The network conversion process in this research utilizes NEXTA, an open-source tool described earlier in the review of literature. NEXTA converts the network shape files 
that are exported from regional demand models to network inputs accepted by other modeling tools. A configuration "import_GIS_Setting.csv" file is needed to map field names between the shape files and the NEXTA format, which includes a series of CSV files.

The demand matrices can also be converted to the required formats using NEXTA. Similar to the configuration file for the network, the "input-demand-metadata.csv" file is used by NEXTA to read and interpret the trip tables exported from the regional demand model or other sources. The demand configuration file in NEXTA needs to be provided with information related to different demand types and demand time periods. To summarize, a configuration file in the NEXTA allows a user to change the network and demand files. There are two different ways to define the demand inputs including time-fixed demand matrix with starting time and ending time and timedependent demand matrices (e.g. 15-30 minutes).

\subsubsection{Supply Calibration}

Calibration of simulation and DTA tools is a complex process that requires examining the results of the models in relation to real-world data and iterations of adjustments of demand, supply, and assignment input parameters. The type of supply side parameters depend on the level of the model. Supply calibration of macroscopic and mesoscopic models considers estimating the capacity, jam density, free-flow speeds, and traffic flow model (TFM) parameters. These parameters are evaluated based on network performance by comparison with the real world measures. The parameters of TFM can be calibrated by fitting pre-defined curves to real-world data using different methods of 
regression and optimization. TFMs in macroscopic and mesoscopic models are based on empirical curves that map the density to flow or speed to volume/capacity ratio. For microscopic models, the calibration parameters are related to individual drivers and vehicles such as lane-changing, car-following, gap acceptance behaviors, and vehicle type and performance (Shabanian, 2014).

Various statistical performance measures are calculated to assess the quality of the calibration and the degrees of deviation between simulated and real-world measures. Available measures include the Mean Absolute Error (MAE), Mean Absolute Percentage Error (MAPE), Root-Mean-Square Error (RMSE), and R-squared $\left(R^{2}\right)$. Different measures describe different aspects of calibration results. RMSE is a measure of the differences between simulated values and the real-world measures. MAE is defined in terms of absolute values of differences between the simulated and real-world measures, and MAPE is defined as the absolute percentage of the differences between the simulated and real-world measures. Compared to MAPE, RMSE gives more weight to the terms with larger differences by using a second order of the differences. Another important measures that is used in this study to assess the simulated values in comparison to realworld measures is R-Squared, which is the measure reflecting how close the simulated results are compared to actual real-world data when fitting a regression line between these two sets of values. Following equations are the expressions for these measures.

$$
\begin{aligned}
& M A E=\frac{1}{N} \sum_{t}\left|P_{t}-P_{t, a}\right| \\
& M A P E=100 \frac{1}{N} \sum_{t}\left|\frac{P_{t}-P_{t, a}}{P_{t, a}}\right| \\
& R M S E=\sqrt{\frac{1}{N} \sum_{t}\left(P_{t}-P_{t, a}\right)^{2}}
\end{aligned}
$$




$$
R^{2}=1-\frac{\sum_{t}\left(P_{t}-P_{t, a}\right)^{2}}{\sum_{t}\left(P_{t, a}-\overline{P_{a}}\right)^{2}}
$$

Where $P_{t}$ is the estimated performance measures based on the simulation at time interval t, $P_{t, a}$ is the corresponding real-world measures, and $\bar{P}_{a}$ is the mean of the realworld measures $\left(P_{t, a}\right) . \mathrm{N}$ is the total number of the time intervals (Lyman and Longnecker, 2001).

\subsubsection{Trip Demand Estimation}

The time-variant trip matrix estimation is an important step in the assignment process. DTA requires trip matrices specified for short time intervals (e.g., 15 minutes or 30 minutes). The derivation of these matrices is one of the most challenging aspects of DTA. Most of the time, these matrices are derived based on initial demand matrices that had been estimated by regional demand models for longer periods of time. Other models are time-of-day models that produce trip matrices by peak period. Either way, these demand matrices have to be converted to time-variant trip matrices. The demand calibration or estimation aims to estimate the trip tables for short intervals (15-minute intervals in this case) based on an initial matrix obtained from the regional demand model. The resulting matrices, when loaded onto the calibrated network, should be able to replicate the observed link volume and congestion pattern (Shabanian, 2014).

An ODME process is used in this study to estimate the time variant matrices starting with the peak period regional model, while attempting to reduce the difference between the link counts and simulated volumes. In this research, the ODME modules in existing DTA tools are used in the estimation. These modules require field data from sensor data, which should be defined and prepared before the ODME process. The 
ODME modules based on the DTA in Cube, DTALite, and VISUM are compared in terms of their ability to replicate real-world data.

\subsubsection{Modeling Tools and Methods}

As illustrated in Figure 3-3, transportation system modeling tools can be classified as different levels including sketch planning, analytical, macroscopic, mesoscopic, and microscopic simulation tools in the modeling of ML. In this study, the following tools are used and their results are compared to model ML.

- $\quad$ ELToD developed by Florida's Turnpike Enterprise (FTE), which has an analytical traffic flow model and static assignment (FTE, 2012).

- $\quad$ DTALite developed by Zhou and his group at Arizona State University, which is an open-source mesoscopic simulation-based DTA tool (Zhou and Taylor, 2012).

- $\quad$ VISUM provided by the PTV Group, which has a macroscopic traffic flow modeling tool with static and dynamic traffic assignment (PTV Vision VISUM 14, 2013).

- Cube Avenue from Citilabs, which is a mesoscopic DTA tool (Citilabs, 2013).

The tools listed above have already been summarized in Appendix 1 and Appendix 2 to use in the modeling of ML.

\subsection{Application of MRM to ML}

To achieve the effective modeling of ML, the MRM approach described in the previous section is applied and evaluated in this study utilizing different tools and 
modeling parameters. The following descriptions are the assignment parameters that are investigated in this study. The remaining subsections describe the different aspects of ML modeling, which are considered in this research.

Figure 3-3 shows the components of this framework that are used in this study for the purpose of ML modeling.

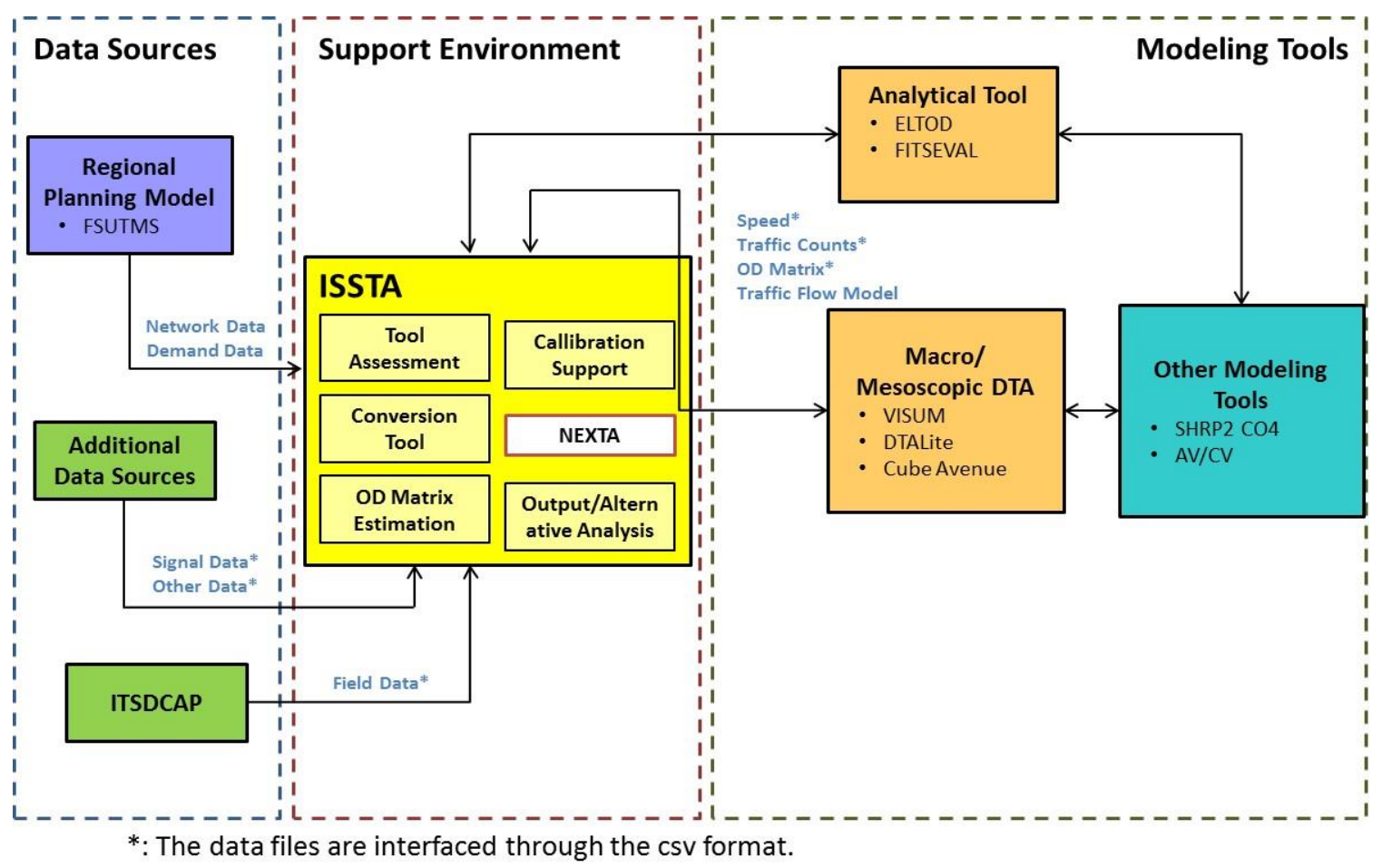

Figure 3-3 Multi-Resolution Modeling (MRM) for Managed Lanes (ML)

\subsubsection{Generalized Cost Function}

For modeling of ML in this study, the generalized cost function used in the assignment process involves the VOT, VOR, and toll costs. To determine the impact of incorporating the VOR in the analysis, only the toll costs and VOT are initially included in the assignment. In this case, the generalized cot function can be expressed in Equation 3-5. If the summation of route travel time and the added equivalent time is still smaller than the congested time in GPL, ML is more attractive to the user. In this 
approach, selecting the route is governed by the user equilibrium assignment procedure, based on the generalized costs of different paths.

$$
U=\text { 回 } a_{1} T T+a_{2} T C
$$

where

$U=$ ? Utility function for route choice, $\mathrm{f}$ (time and cost),

$T T=$ Estimated travel time (minute),

$T C=$ 回 Toll cost (dollar),

$a_{1}=$ Travel time coefficient, and

$a_{2}=$ Toll cost coefficient.

The parameter $a_{1}$ in the above equation is related to the value of time. A value of time of $\$ 30$ per hour means that the user pays $\$ 30$ to save one hour, or 50 cents for every minute of saved time. In most current static and dynamic assignment applications, an average VOT is used and the variability in user's perception of VOT is ignored. The variability is expected due to different socio-economic user categories, the variation of users within each category, and the variation of user attributes between days. However, a distribution of VOT, possibly combined with a categorization of users by income and/or other attributes, can better capture the preference of different road users for different traffic conditions. In the SHRP 2 C04 project, a lognormal distribution is assumed for the distribution of the value of time, as shown in Figure 3-4 . In this figure, given a toll value of $\$ 20$, the proportion of payers shown in the blue area are the people that have VOT savings exceeding (SHRP 2 C04, 2013). 


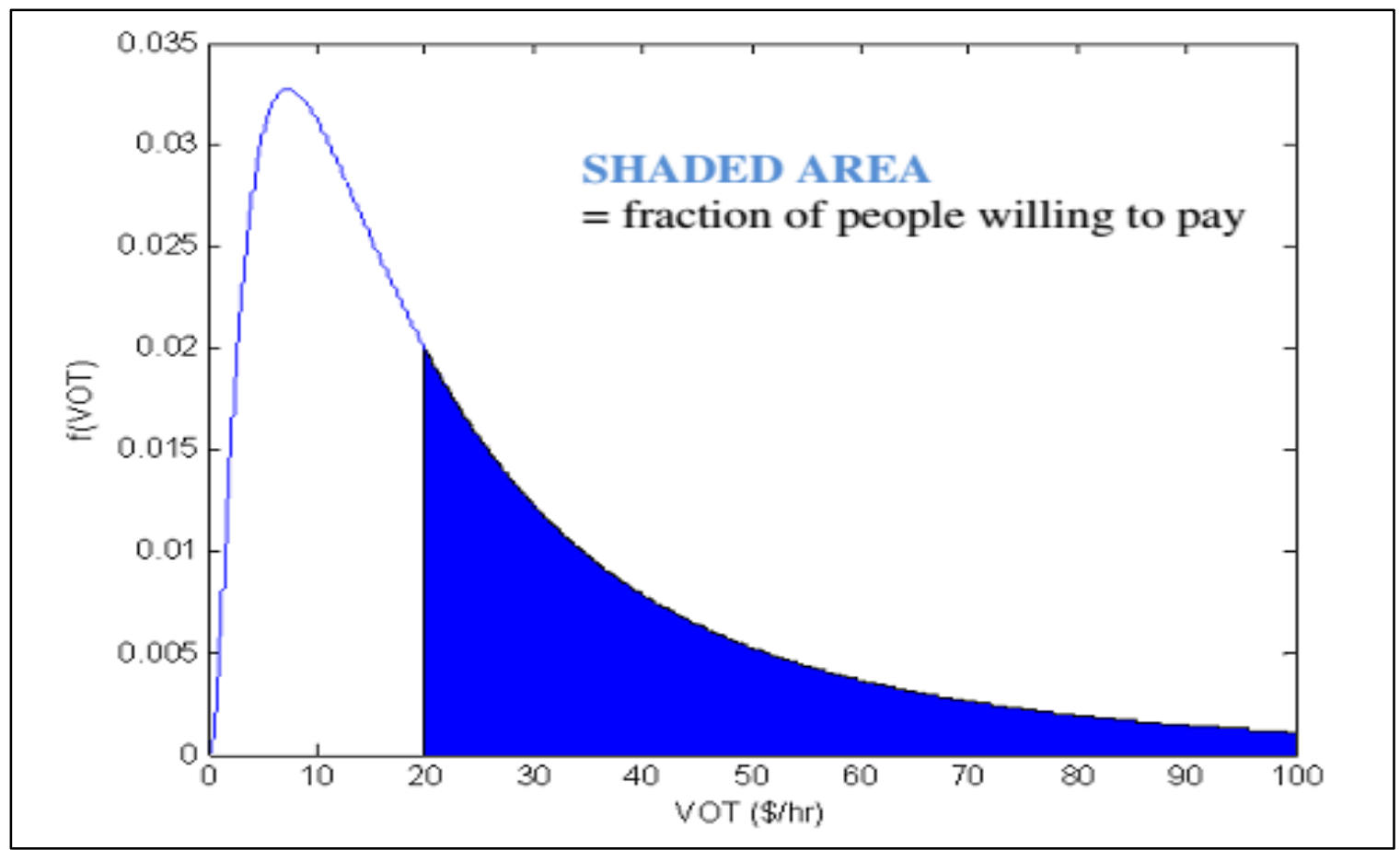

Figure 3-4 Lognormal Distribution for VOT Based on SHRP 2 C04 Project (SHRP 2 C04, 2013)

With the same average travel time for two different alternative routes, drivers generally prefer the more reliable alternative with the least day-to-day variability in travel time. However, travel time reliability has not been sufficiently considered in previous managed lane modeling. One of the important contribution of this project is to develop a function that estimates the reliability for inclusion in the generalized cost function of the assignment. in the in the modeling of ML to assess the impact of travel time variability in the diversion to the ML. Measuring reliability requires to be translated into measures represented by the $80^{\text {th }}$ or $95^{\text {th }}$ percentile of travel time indices versus the median. In this study, a methodology is proposed to incorporate the impacts of travel time reliability in the selection of managed lanes.

The methodology uses the general function proposed in the SHRP 2 L03 project (Margiotta et al., 2013). The parameters of the function for the case study of this project 
(I-95 northbound corridor in Miami) were developed using the regression analysis proposed in the SHRP2 L38C project. The SHRP 2 L38C project developed the regression equation that estimates travel time reliability as functions of demand/capacity (d/c) ratio, lane hour lost due to incidents, and weather conditions as shown in Equation 3-6 (Hadi et al., 2014):

$$
T T I_{n \%}=e^{b 1 d c_{c r i t}+b 2 L H L+b 3 R_{0.05}+b 4}+b 5 \times \text { Length }+b 6 \times \text { Length }^{2}+b 7
$$

where

$$
\begin{aligned}
& T T I_{n \%}=\mathrm{n}^{\text {th }} \text { percentile TTI, } \\
& L H L=\text { Lane-hour lost, } \\
& d c_{\text {crit }}=\text { Critical demand-capacity ratio, } \\
& R_{0.05^{\prime \prime}}=\text { Hours of rainfall exceeding } 0.05 \text { inch, } \\
& \text { Length }=\text { Segment length }(\mathrm{mi}), \text { and }
\end{aligned}
$$$$
b_{1}, b_{2}, b_{3}, b_{4}, b_{5}, b_{6}, \text { and } b_{7}=\text { Coefficients for } \mathrm{n}^{\text {th }} \text { percentile TTI. }
$$

According to this equation, $\operatorname{TTI}_{n} \%$ is the $n^{\text {th }}$ percentile travel time indices. The TTI is the ratio of the travel time at the specific percentile to the free flow travel time for the study segment. Then, the travel time of $80^{\text {th }}$ and $95^{\text {th }}$ percentiles are calculated. Following descriptions are the variables used in the equation based on SHRP 2 L38C (Hadi et al., 2014):

- 'Lane hour lost: The average number of lanes blocked per incident or work zone multiplied by the average duration of blockage and the total number of incidents or work zones during the time interval. 
- Critical demand-capacity ratio $\left(\mathbf{d c}_{\text {crit }}\right)$ : The ratio of demand to capacity during the most critical hour of the study period.

- Hours of rainfall exceeding 0.05 inch $\left(\mathbf{R}_{0.05}\right.$ "). The hours of rainfall when exceeding 0.05 inch during the study period."

Table 3-1 Coefficients for Different TTI Percentile (Hadi et al., 2014)

\begin{tabular}{|c|c|c|c|c|c|c|c|c|}
\hline Percentile $^{\text {th }}$ & $\boldsymbol{R}^{\mathbf{2}}$ & $\boldsymbol{b}_{\mathbf{1}}$ & $\boldsymbol{b}_{\mathbf{2}}$ & $\boldsymbol{b}_{\mathbf{3}}$ & $\boldsymbol{b}_{\mathbf{4}}$ & $\boldsymbol{b}_{\mathbf{5}}$ & $\boldsymbol{b}_{\mathbf{6}}$ & $\boldsymbol{b}_{\mathbf{7}}$ \\
\hline $\mathbf{1 0}$ & 0.561 & 0.271 & 0.001 & 0.009 & -0.952 & 0.034 & -0.013 & 0.541 \\
\hline $\mathbf{5 0}$ & 0.693 & 14.000 & 0.001 & 0.003 & -14.681 & -0.452 & 0.081 & 1.511 \\
\hline $\mathbf{8 0}$ & 0.704 & 12.802 & 0.002 & 0.003 & -12.991 & -0.782 & 0.141 & 1.882 \\
\hline $\mathbf{9 5}$ & 0.741 & 10.763 & 0.001 & 0.004 & -10.433 & -0.632 & 0.092 & 1.862 \\
\hline $\mathbf{9 9}$ & 0.762 & 6.792 & 0.001 & 0.003 & -5.864 & -0.591 & 0.053 & 1.992 \\
\hline Mean & 0.762 & 12.103 & 0.001 & 0.002 & -12.631 & -0.411 & 0.071 & 1.512 \\
\hline
\end{tabular}

To add the reliability and associated values in the generalized cost function, Equation 3-7 is utilized to incorporate the travel time $80^{\text {th }}$ and $95^{\text {th }}$ percentiles as estimated from Equation 3-6.

$$
U=a_{1} T T+a_{2} T C+a_{3} T T_{80 \%}+a_{4} T T_{95 \%}
$$

where

$U=$ Utility function for route choice, $\mathrm{f}$ (time, cost, reliability),

$T T=$ Estimated travel time (minute),

$T C=$ Toll cost (dollar),

$a_{1}=$ Travel time coefficient,

$a_{2}=$ Toll cost coefficient,

$\mathrm{a}_{3}=$ Coefficient for reliability measure $\left(80^{\text {th }}\right.$ percentile of travel time $)$, and

$a_{4}=$ Coefficient for reliability measure $\left(95^{\text {th }}\right.$ percentile of travel time $)$. 
As explained in the review of literature, the VOR represents travelers' willingness to pay for the reduction in travel time variability. The SHRP2 C04 project evaluated the reliability ratio (VOT/VOR) for an average trip distance and found that the ratio is in the range of 0.7 to 1.5 based on a stated preference (SP) survey conducted in that project (SHRP 2 C04, 2013). In this study, a value of 1.1 is assumed for the ratio VOT/VOR, which is corresponding to $\mathrm{a}_{1} /\left(\mathrm{a}_{3}+\mathrm{a}_{4}\right)$, and $\mathrm{a}_{1}$ is calculated according to the following equation:

$$
a_{1}=1.1\left(a_{3}+a_{4}\right)
$$

Where $a_{3}$ is assumed to be equal to $a_{4}$ assuming that travelers put the same weight on the $80^{\text {th }}$ and $95^{\text {th }}$ percentile travel time. This method requires an inclusion of an additional reliability term in the mode choice model and the use of Equation 3-6 and Equation 3-8 to estimate the reliability. However, it also requires an estimation of the LHL and $\mathrm{R}_{0.05^{\prime}}$ to estimate the $80^{\text {th }}$ and $95^{\text {th }}$ percentile of travel time indices.

\subsubsection{Dynamic Pricing}

In emulating the real-world in the DTA models, the toll is updated for each interval, based on the maximum density of the ML so as to preserve the desired level of service in ML, as is done in the real-world toll schedule. In this approach, the DTA assignment computes the link density (veh/mi/ln) for each time segment by dividing the hourly assigned volumes per lane by the link speed (mi/hr), as follows:

$$
\text { Link Density }(\text { veh } / \mathrm{mi} / \text { ln })=\frac{\text { Hourly link Volumes }(\text { veh } / \text { ln } / \mathrm{hr})}{\text { Link Speed }(\mathrm{mi} / \mathrm{hr})}
$$

To achieve dynamic pricing in the modeling of ML, a script language in Cube Avenue was developed in this study to code the link density (veh/mi/ln) for each time 
segment and, to identify the largest density on the ML by comparing the link densities for all of the links of the ML for each direction. Once the largest density is found at the end of each time segment, the toll cost (dollar) is obtained from an input lookup table based on the values used in different scheduled toll policies.

\subsection{Assessing the Impacts of Advanced Vehicle Technology Utilizing MRM to ML}

\subsubsection{Capacity Impact Estimation based on Microscopic Simulation}

As stated earlier, the lane capacity as a function of the proportions of ACC/ CACC vehicles was estimated by Shladover et al. (2012), using microscopic sim0ulation to estimate the effect of different market penetrations of ACC and CACC on freeway capacity. The distribution of time gap settings that drivers used in a real field test was used in the simulation. In the study conducted by Shladover et al. (2012), it was necessary to develop and validate several mathematical models such as car-following logic for each operational mode, merging of vehicles entering from an on-ramp, and free driving of a vehicle in uncongested condition.

As implied from the first bullet above, each of the three vehicle operational modes (manual driving, ACC, and CACC with platooning) has its own following logic. The parameters of the used car-following models are the current distance between vehicles, speeds of both the preceding and following vehicles, and vehicle lengths. The simulation tool that they used to assess the capacity impacts was AIMSUN, which is a microscopic simulation tool.

The maximum flow for manually driven vehicles on a simulated freeway link was assumed to be about 2,200 veh/ln/hr. Thus, the minimum time headway for manual 
driving was estimated to be $1.64 \mathrm{sec}(\approx 3,600 / 2,200 \mathrm{veh} / \mathrm{ln} / \mathrm{hr})$. The desired time gaps of the ACC or CACC vehicles were selected from the gaps actually selected by drivers in the field test as listed below:

- $\quad$ ACC: $31.1 \%$ at $2.2 \mathrm{sec}, 18.5 \%$ at $1.6 \mathrm{sec}, 50.4 \%$ at $1.1 \mathrm{sec}$

- $\quad$ CACC: $12 \%$ at $1.1 \mathrm{sec}, 17 \%$ at $0.9 \mathrm{sec}, 24 \%$ at $0.7 \mathrm{sec}$, and $57 \%$ at $0.6 \mathrm{sec}$.

The simulation results showed a narrow range of maximum flow rate (between 2,018 veh/ln/hr and 2,100 veh/ln/hr) with different market penetration of ACC. When the percentage of ACC increased to $80 \%$, the maximum flow rate increased to just about $2100 \mathrm{veh} / \mathrm{ln} / \mathrm{hr}$. The increase in the percentage of ACC vehicles to more than $80 \%$, actually led to a small decrease in capacity to $2,054 \mathrm{veh} / \mathrm{ln} / \mathrm{hr}$, because of the longer time gap of the ACC compared to manual driving. Table 3-2 shows the simulation results for the capacity estimates with different market penetrations of ACC vehicles.

Table 3-2 Simulation Results for Different Percentage of ACC (Shladover et al., 2012)

\begin{tabular}{|c|c|}
\hline Percentage of ACC Vehicles (\%) & Lane Capacity (veh/ln/hr) \\
\hline 0 & 2000 \\
\hline 20 & 2070 \\
\hline 40 & 2094 \\
\hline 60 & 2094 \\
\hline 80 & 2100 \\
\hline 100 & 2054 \\
\hline
\end{tabular}

Table 3-3 presents the capacity estimates with different percentages of CACC vehicles in the traffic stream. It was shown by Shladover et al. (2012) that the lane capacity increased significantly from $2,018 \mathrm{veh} / \mathrm{ln} / \mathrm{hr}$ to $4,000 \mathrm{veh} / \mathrm{ln} / \mathrm{hr}$ as the market penetration of $\mathrm{CACC}$ vehicles increased from $0 \%$ to $100 \%$. At $20 \%$ market penetration, the lane capacity increased by $3 \%$; at $40 \%$ market penetration, the capacity increased more than $10 \%$; and at $60 \%$ market penetration, the increase in the capacity is about $23 \%$. 
Table 3-3 Simulation Results for Different Percentage of CACC (Shladover et al., 2012)

\begin{tabular}{|c|c|}
\hline Percentage of CACC Vehicles (\%) & Lane Capacity (v/hr/ln) \\
\hline 0 & 2018 \\
\hline 20 & 2092 \\
\hline 40 & 2230 \\
\hline 60 & 2500 \\
\hline 80 & 2890 \\
\hline 100 & 4000 \\
\hline
\end{tabular}

Finally, Shladover et al. (2012) tested the effects of different combinations of ACC and CACC market penetrations with the remaining vehicles manually driven. Table 3-4 shows the impacts of the combination of ACC and CACC with different market penetration on the lane capacity.

Table 3-4 Estimation of Lane Capacity for ACC and CACC Vehicles at Time Gaps Chosen by Drivers in Field Data with the remaining vehicles manually driven (Shladover et al., 2012)

\begin{tabular}{|c|c|c|c|c|c|c|c|c|c|c|}
\hline \multirow{11}{*}{ 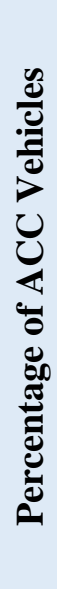 } & \multicolumn{10}{|c|}{ Percentage of CACC Vehicles } \\
\hline & & $10 \%$ & $20 \%$ & $30 \%$ & $40 \%$ & $50 \%$ & $60 \%$ & $70 \%$ & $80 \%$ & $90 \%$ \\
\hline & $10 \%$ & 2065 & 2090 & 2170 & 2265 & 2389 & 2458 & 2662 & 2963 & 3389 \\
\hline & $20 \%$ & 2065 & 2110 & 2179 & 2265 & 2378 & 2456 & 2671 & 2977 & 0 \\
\hline & $30 \%$ & 2077 & 2127 & 2179 & 2269 & 2384 & 2487 & 2710 & 0 & 0 \\
\hline & $40 \%$ & 2088 & 2128 & 2192 & 2273 & 2314 & 2522 & 0 & 0 & 0 \\
\hline & $50 \%$ & 2095 & 2133 & 2188 & 2230 & 2365 & 0 & 0 & 0 & 0 \\
\hline & $60 \%$ & 2101 & 2138 & 2136 & 2231 & 0 & 0 & 0 & 0 & 0 \\
\hline & $70 \%$ & 2110 & 2084 & 2155 & 0 & 0 & 0 & 0 & 0 & 0 \\
\hline & $80 \%$ & 2087 & 2101 & 0 & 0 & 0 & 0 & 0 & 0 & 0 \\
\hline & $90 \%$ & 2068 & 0 & 0 & 0 & 0 & 0 & 0 & 0 & 0 \\
\hline
\end{tabular}

3.3.2. Modeling of CACC Vehicle on Managed Lanes Based on Macroscopic and

\section{Mesoscopic Simulation}

The results from microscopic simulation, as described above, confirm that increasing in market penetration of CACC significantly increases capacity. The market penetration of CACC technology is expected to increase in the next 25 years until reaching $100 \%$, at a rate that depends on the prediction model and the associated assumptions. If preferential treatment is given to CACC vehicles when using ML, this 
can result in an increase in their percentage on ML and it is expected that the mobility benefits of these devices will be achieved sooner. One important contribution of this study is to illustrate how a multi-resolution approach can be used to assess advanced vehicle technology on the performance measurement of ML. For this purpose, this study assesses the impacts of CACC vehicle technologies on ML, GPL, and overall system performance including alternative routes using macroscopic and mesoscopic models based on capacity estimates from microscopic simulation models. The assessment is based on the capacity estimated by Shladover et al. (2012), as described in the previous section. This capacity is coded as a variable in the demand forecasting modeling tool with macroscopic traffic model, and a mesoscopic simulation-based DTA tool. The capacity is allowed to vary in each assignment iteration, as a function of the percentages of CACC in traffic streams in that iteration, according to the findings from the microscopic simulation study.

This approach demonstrates the power of MRM in the modeling of CACC vehicle with the capacity estimated using microscopic simulation and mesoscopic simulationbased DTA to assess the sub-area impacts and diversions between ML, GPL, and other alternative routes considering preferential treatments of equipped vehicles. For each percentage of these technologies, this research compares the mobility benefits based on the traffic flow models in the utilized tools. Attempts are also made to model the impacts of CACC based on the regional level of modeling utilizing Cube Voyager.

The DTA tools used in this component of the research should accept different user classes in different facility types and should also be able to vary the capacity dynamically based on the proportions of the user classes. These capabilities should allow 
different CACC market penetration scenarios, different pricing schedules, and generalized cost function to be applied to different user groups. First, an attempt is done to model the impacts of CACC in Cube Voyager (Citilabs, 2013), which is a demand forecasting modeling tool with a macroscopic traffic model and static traffic assignment (STA). The CACC-equipped vehicle is coded as a user group with origin-destination demands estimated based on each investigated market penetration of connected vehicles.

The scripting language of the Cube software is used to code the capacity as a variable that is a function of the CACC proportion on each facility, as determined during the assignment iterations, the script language in Cube is also used to implement different tolling strategies on the ML for different user groups including single occupancy vehicles, high occupancy vehicles, and CACC-equipped vehicles on ML, as described later in this section. The tolling algorithm, implemented in the script language, is dynamic and changed with the congestion level on ML. This algorithm emulates the pricing algorithm currently used to set the pricing dynamically on the I-95 Express ML in Miami, FL, which is the case study in this research.

In this script to model ML with different user groups, the approach involves adding the toll cost to the generalized utility function of the link for use in the assignment process that determines the diversions to/from ML. In this study, four user groups and their demand matrix are obtained from the regional demand forecasting model: Drive Alone (DA), Shared Ride of 2 occupants (SRP2), Shared Ride of three or more occupants (SRP3), and Truck. According to the I-95 Express tolling strategy, the DA and SRP2 vehicles pay the same toll to access ML. Therefore, the DA and SRP2 matrices are grouped together and are referred to as Tolled Vehicles (TV) in the assignment module. 
As with the real-world implementation of I-95 Express, the script is written to allow SRP3 vehicles to use the ML without any cost or restriction, and trucks are not allowed to use the ML. Different market penetrations of CACC vehicles are investigated by creating new user groups with their own O-D matrices based on the market penetration. To emulate real-world conditions, as much as possible, the O-D demands are estimated and assigned for each 15-minute period. The estimation is based on initial O-D matrices for the sub-area extracted from the demand forecasting model, and is adjusted utilizing the ODME procedure based on real-world counts collected from multiple sources.

The static assignment of Cube Voyager is then conducted by running the assignment for each of the 15-minute trip tables. The output files contain volumes and speeds for each time intervals that are combined and used to assess the impacts of CACC and the associated ML tolling strategies. It should be mentioned, however, that the runs with the 15-minute demands are independent from each other, and the run for one interval is not affected by the results of the previous interval because static assignment is not capable of modeling these interactions between time intervals. In addition, the utilized traffic flow models normally used in demand forecasting models are less accurate, particularly under congested and queuing conditions, which may affect the results. This is addressed in this study by using mesoscopic simulation based-DTA modeling, as described below.

To consider the dynamic interaction between traffic assignment and system performance under congested conditions, this study uses a mesoscopic simulation-based DTA to model different CACC market penetration and ML strategy combinations. To accomplish this, there is a need for a toll that allows varying the capacity dynamically 
based on the proportions of CACC vehicles in the traffic stream, as described earlier with the static assignment. The mesoscopic simulation-based DTA tool, Cube Avenue (Citilabs, 2013), satisfies this requirement since it allows the use of the Cube script language to vary the speed in each iteration, in a similar manner to what described earlier for static assignment. This DTA tool also allows the coding of different user groups with different ML preferential treatments, pricing schedules, and generalized cost functions, as is done in the static assignment of Cube Voyager.

A script language is created for the execution of the mesoscopic simulation-based DTA, in a similar approach to the one used in the static assignment modeling described earlier. However, instead of running the STA module for 12 consecutive periods of 15minute intervals as had to be done with the STA, the DTA is run for the whole modeling period as allowed by DTA. Obviously, this procedure is expected to produce superior results compared to the STA, because the DTA and associated simulation models the dynamic interactions between assignment and traffic flow performance including the consideration of queues and queue spillbacks in subsequent time intervals. Different toll pricing schedules for different percentages of CACC vehicles are tested to assess the impacts on the diversion rate between ML and GPL and the performance of these facilities.

\subsection{Summary}

Advanced modeling strategies require advanced methods and tools that work together to deliver the required results. The methodology presented in this chapter 
suggested integrating the components of the methods and tools into a single MRM framework.

This study recommended the selection of the modeling tools based on the comprehensive criteria. For this purpose, an assessment was done to select and use the modeling tools in this research to meet the modeling requirements in the MRM framework. There is also a need for the selection of the best parameters of the traffic flow models, demand, and assignment. This chapter described how these parameters can be selected and assessed.

The proposed MRM framework was applied and evaluated in this study for modeling ML and the impacts of associated operational and pricing strategies. This chapter also described how the proposed MRM framework can be implemented to a case study that assesses the operations of AVs/CVs on ML. 


\section{CHAPTER 4}

\section{APPLICATION OF DEVELOPED METHODOLOGY}

The methodology described in the previous chapter was applied to a network, which was called the exploration network. The purpose of using the exploration network, which has already been modeled and calibrated with a mesoscopic simulation-based DTA tool (Cube Avenue) by Hadi et al. (2013), was to explore and examine the concepts and the components of the MRM proposed in this study and to select the best concepts and modeling tools for the purposes of this study. Since the exploration network has been examined in detail in a previous study by Hadi et al. (2013), its use yields a good assessment of the methods, tools, and procedures of this study. The remaining parts of this chapter describe the related efforts in this research.

\subsection{Data Processing and Importing to DTA Tools}

The case study used in this research to assess the tools and methods was a subarea around the I-95 corridor in Miami, FL, as shown in Figure 4-1. This network has a total number of 288 nodes, 303 links and 57 zones. Two-lane managed lanes are deployed along the I-95 corridor with a soft barrier separation from parallel general purpose lanes. This relatively small network has been modeled, calibrated, and tested in a mesoscopic simulation-based DTA tool (Cube Avenue) by Hadi et al. (2013), which

allows a better comparison of different managed lane modeling tools and assessment of the developed MRM procedures. 


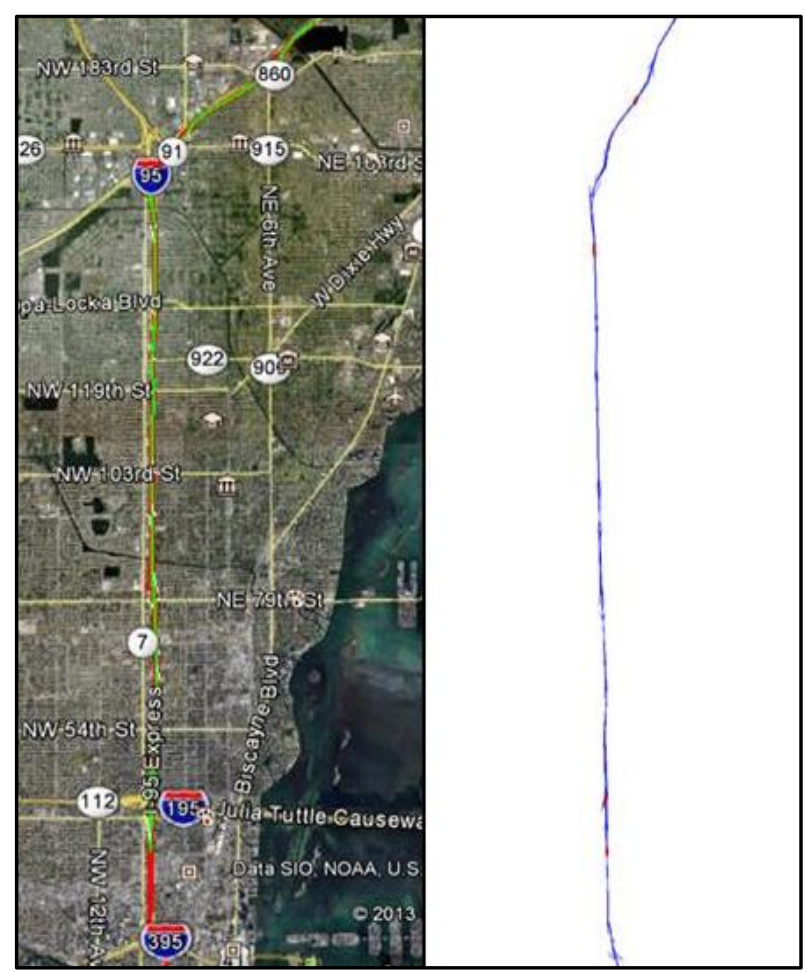

Figure 4-1 Exploration Network, Extracted Subarea from the SERPM Model

A number of modeling tools were used in this study, which includes two macroscopic tools, ELToD coded in Cube and VISUM, and two mesoscopic DTA tools, Cube Avenue and DTALite. As mentioned above, the network has been modeled in the Cube environment in the previous research project conducted by the research team, which provides a basis for modeling the same network in the other two tools, DTALite and VISUM. The following section describes the related efforts for data preparation and model conversion.

\subsubsection{Network and Demand Data Conversion}

\section{Data Conversion to DTALite}

The first step was to create a set of shape files describing the network to be imported to NEXTA from Cube Avenue. The network data structure in these files defines 
the basic node and link structure used in the NEXTA tools, along with the attributes for each link and node.

The node and link shape files exported from the Cube model were imported into NEXTA, the graphical user interface for DTALite, through the network importing function. The corresponding link and node attributes, such as the number of lanes, freeflow speed, link capacity, traffic control type, and so on, were configured in the "Import_GIS_Setting.csv" of NEXTA. All NEXTA data files are in the CSV format. In addition, the link types used in the Cube model are different from those used in DTALite. In Cube, there is no limitation in the number of link types, while a total number of 12 link types are allowed in DTALite. The conversion of link types from Cube to DTALite was conducted based on the matches listed in Table 4-1.

Table 4-1 Required Link Type to Model Traffic Network in DTALite

\begin{tabular}{|l|l|}
\hline \multicolumn{1}{|c|}{ Cube Model } & \multicolumn{1}{c|}{ NEXTA/DTALite } \\
\hline 10-19: Freeway & 1: Freeway \\
20-29: Divided arterial & 2: Highway/Expressway \\
30-39: Undivided arterial & 3: Principal arterial \\
40-49: Collector & 4: Major arterial \\
50-59: Centroid connector & 5: Minor arterial \\
60-69: One-way facility & 6: Collector \\
70-79: Ramps & 7: Local \\
80-89: Exclusive HOV lanes & 8: Frontage road \\
90-99: Toll facilities & 9: Ramp \\
\end{tabular}

It should be noted that the zone layer is not required in the Cube model as the zone centroid information is specified through centroid nodes. However, the zone numbers have to be explicitly specified in NEXTA. In this study, the zone number 
attribute is added to the nodes located at the centroid of the zones. Figure 4-2 shows the final imported network in NEXTA.

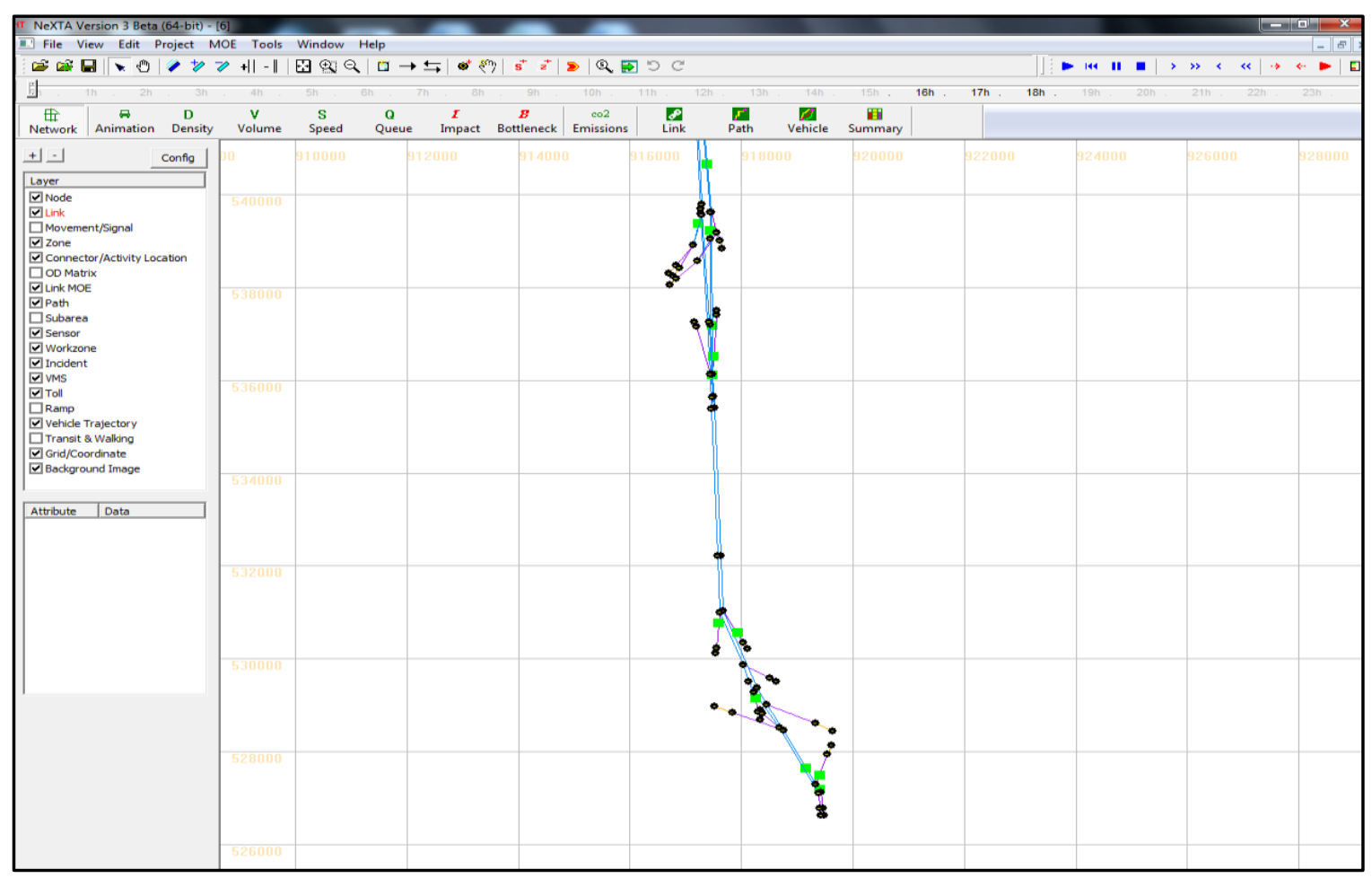

Figure 4-2 Final Imported Network in NEXTA

In addition to the network data, the demand data should be imported into simulation tools to run the assignment model. In this research, the demand matrices from the Cube Avenue model were imported into the other two tools and used as baseline initial matrices in the analysis. It should be mentioned that these demands were derived by Hadi et al. (2013) based on the initial demand matrices extracted from the Southeast Regional Planning model for the peak period, and they were further calibrated using the static Cube Analyst ODME procedure. The Cube demand matrices were converted to the csv file format and imported into NEXTA through the demand meta database configuration file in NEXTA as shown in Figure 4-3. As illustrated in this figure, the user 
has to specify the vehicle type, demand matrix type, and the corresponding time period covered by the matrix.

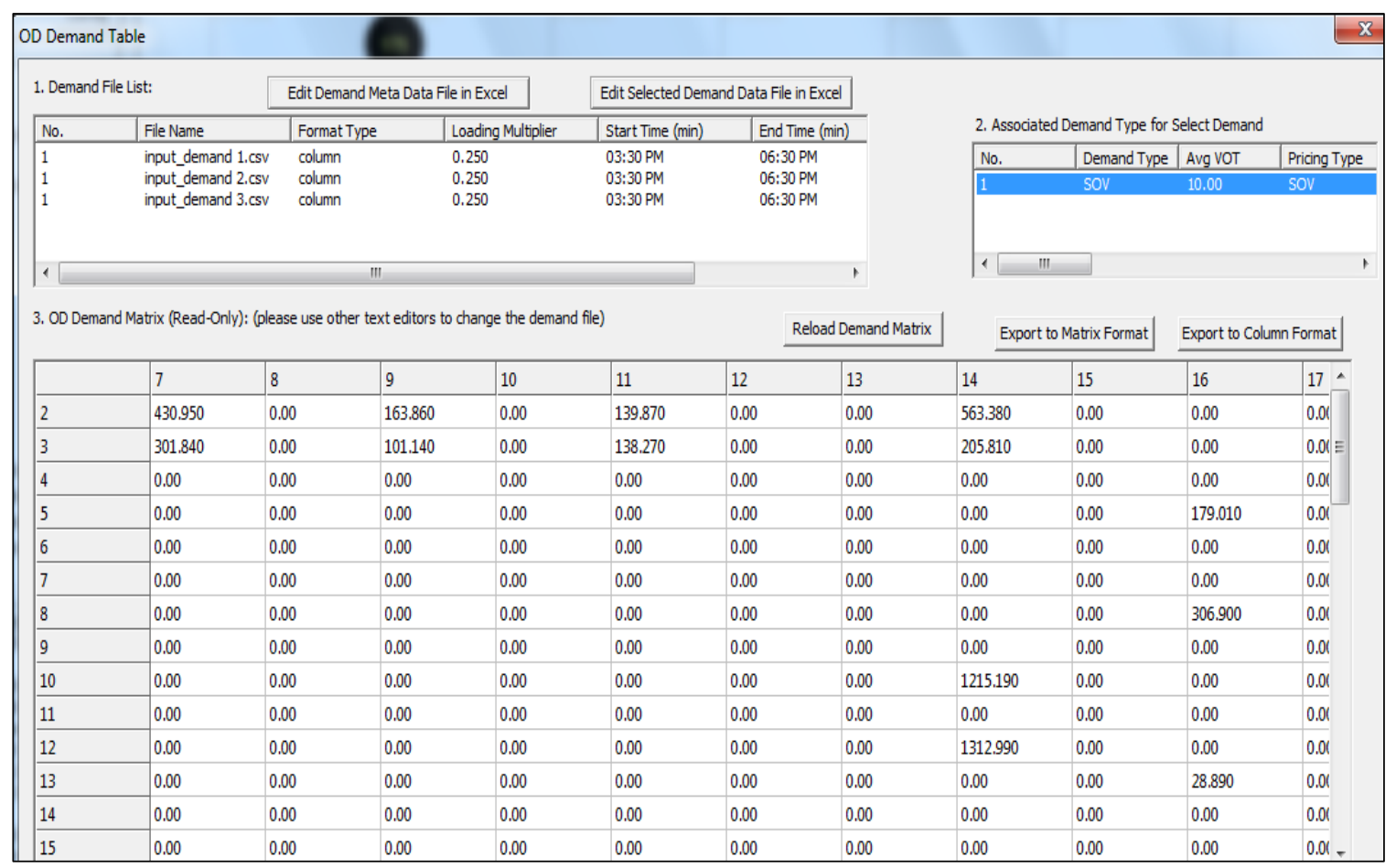

Figure 4-3 Demand Import Interface in NEXTA

Before running the DTALite model, the user needs to configure the scenario setting file, which is the "input_scenario_settings.csv" file. The scenario settings file allows changing the characteristics of the scenarios being run, as well as creating various traffic scenarios that can be run simultaneously. Scenario attributes such as the demand multiplier, traffic flow model parameter, and number of days for running a scenario can be changed in this file. The user can also define different simulation scenarios such as managed lane, work zone, and incident scenarios to be modeled in this file. In this study, a managed lane scenario was created using this function, as shown in Figure 4-4. 


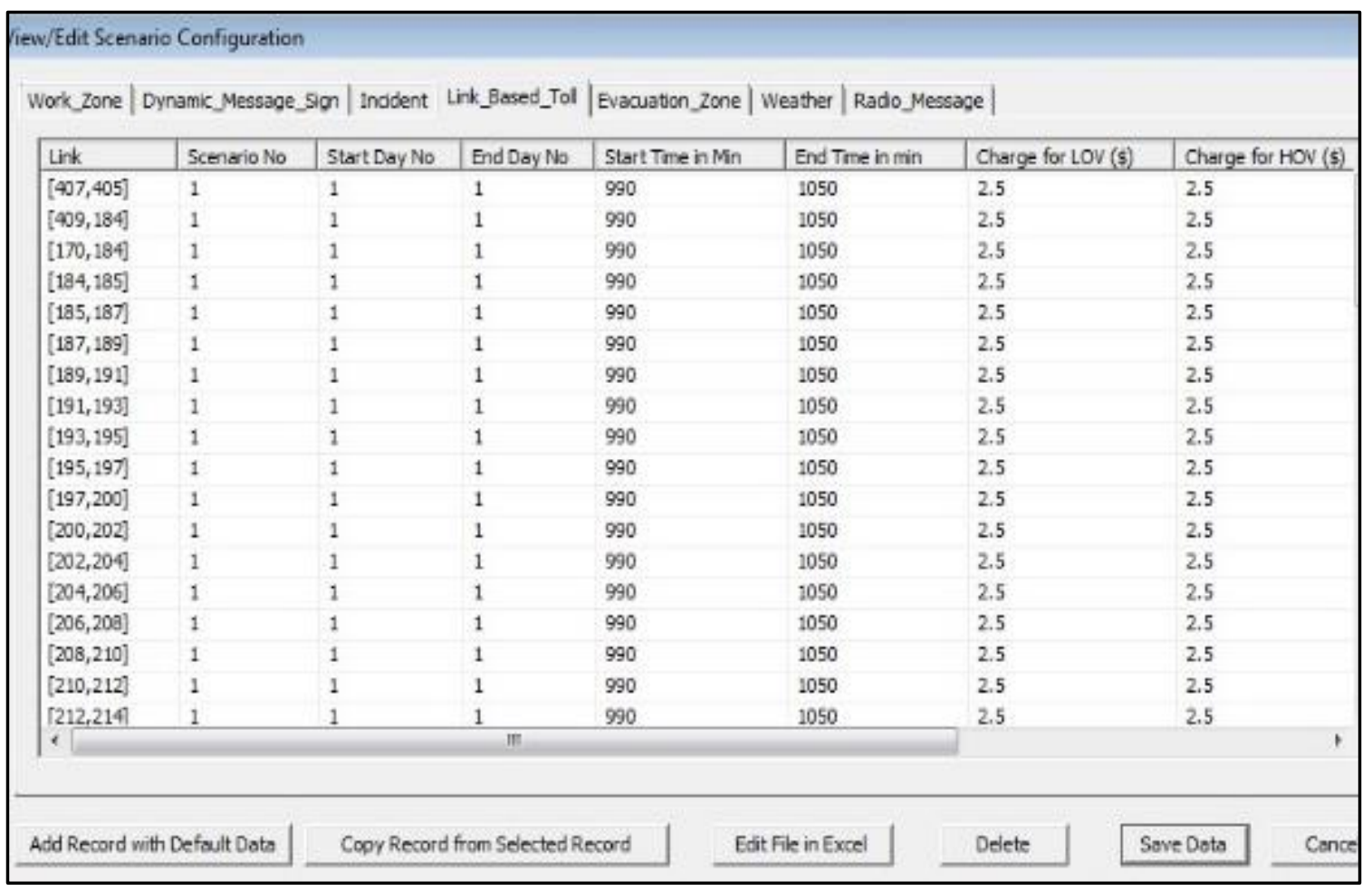

Figure 4-4 Managed Lane Scenario Configuration

\section{Data Conversion to VISUM}

VISUM provides an Add-In feature to import network files from other software such as NEXTA or Cube. However, after applying this function, a further check is still needed to ensure the consistency of the network presentation and data inputs, as different models may have different spatial and temporal resolutions. In this study, the input link types and node types were updated first. The corresponding GIS shape files were prepared based on the format required by VISUM and imported into VISUM. The Cube demand matrices were then converted into csv or excel files and imported into VISUM. Figure 4-5 and Figure 4-6 show the related VISUM functions and the final imported network and demand matrix in VISUM, respectively. 


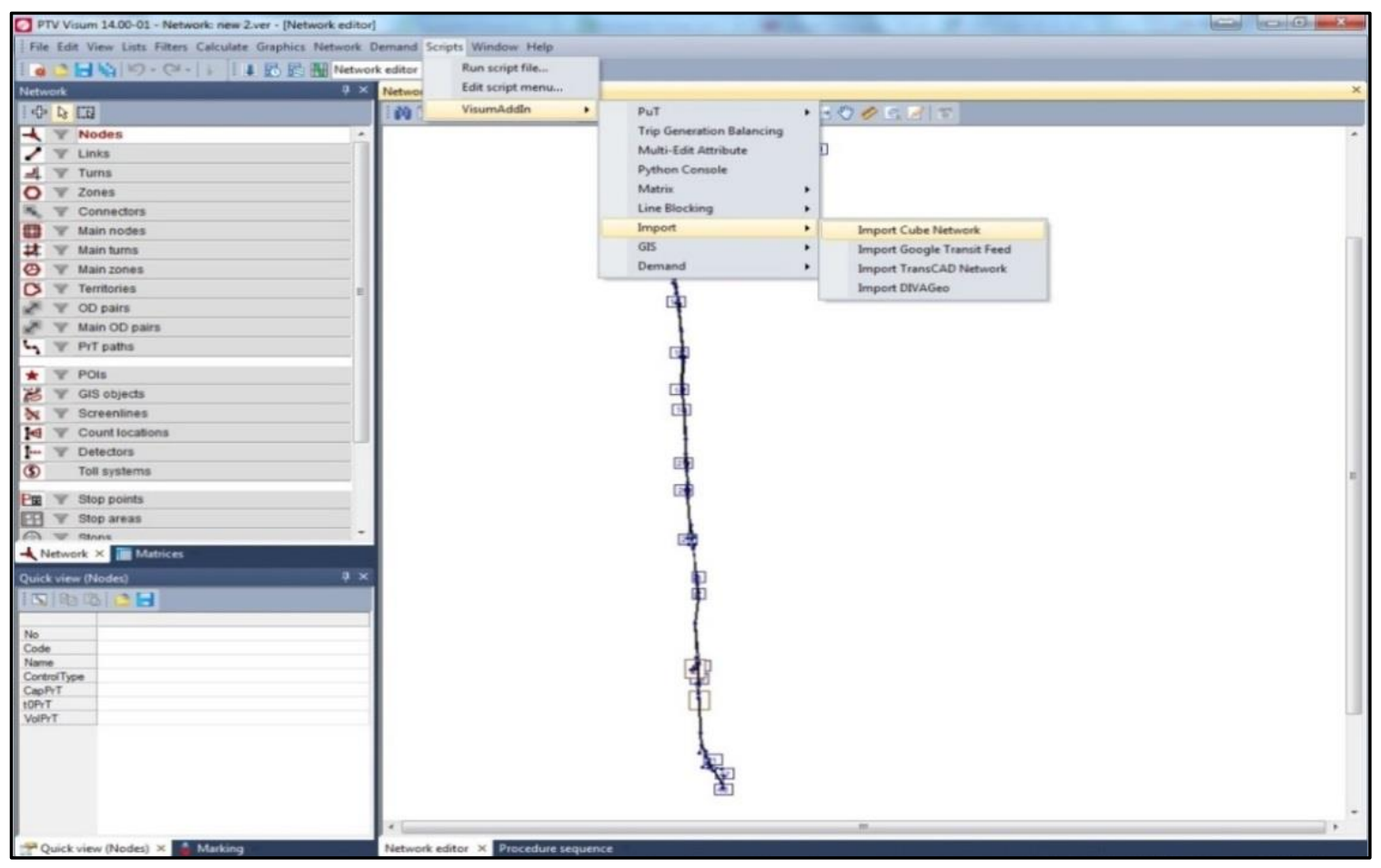

Figure 4-5 Imported Network in VISUM

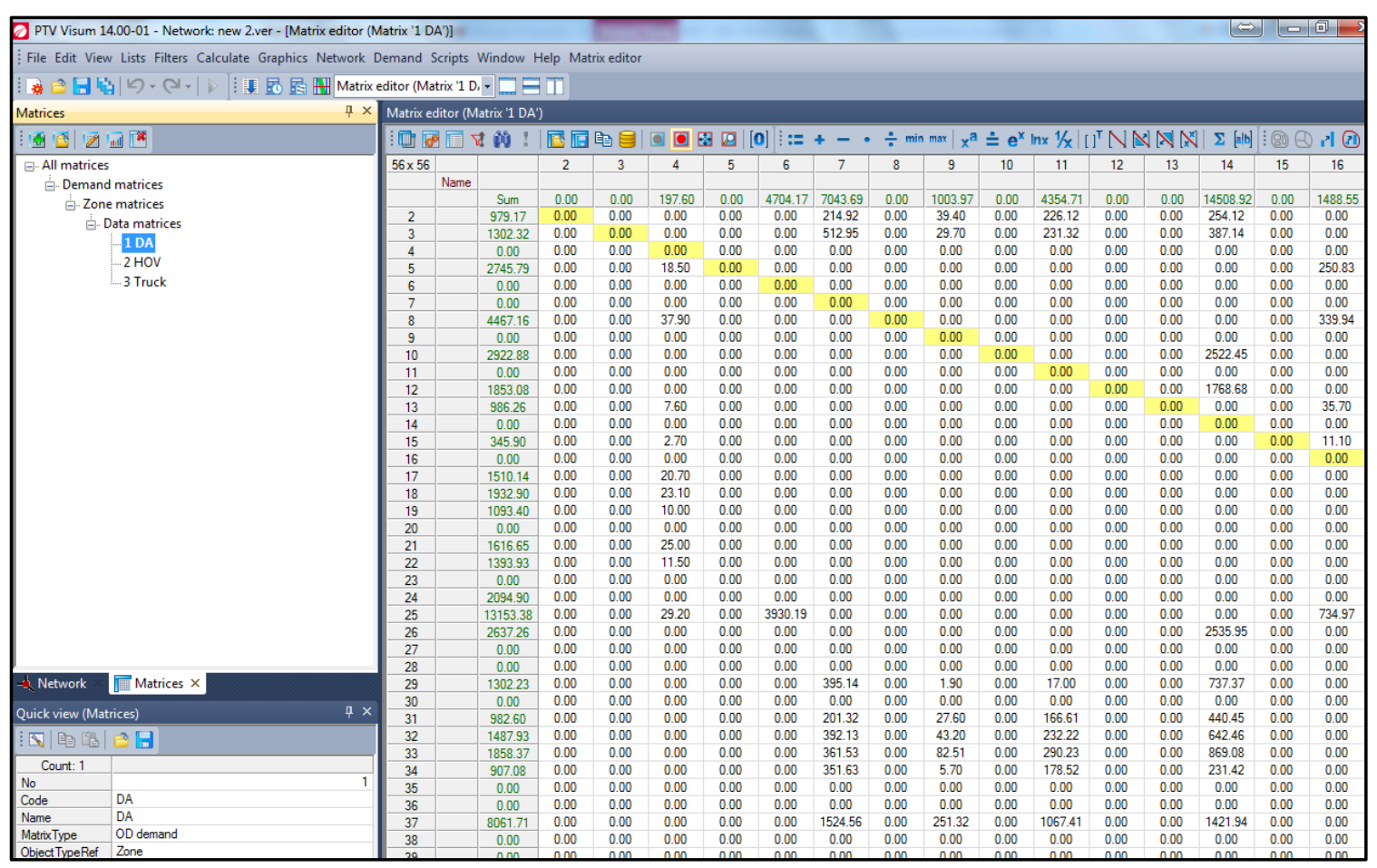

Figure 4-6 Imported Demand Matrix in VISUM 


\subsubsection{Supply Network Calibration}

As explained in the methodology chapter, the network supply calibration estimates the network parameters such as capacity and other traffic flow model (TFM) parameters that define network performance in producing travel time, forming queues, and queue spillbacks.

Different modeling tools have different traffic flow models. For example, the Bureau of Public Road (BPR) function is usually used in the FSUTMS model within the Cube environment although the Akcelik model has also been used. DTALite allows the use of BPR, Spatial Queue Model, and Newell's N-Curve model. VISUM also provides multiple forms of traffic flow models such as Isochrones and Spatial Queue Model. This study examines the impacts of utilizing calibrated and uncalibrated TFM parameters, which are capacity and jam density, on the simulation results using different modeling tools.

The previous study conducted by Hadi et al. (2013) on the segment of I-95 emphasized that only data from congested segments that are not affected by downstream bottlenecks should be used to calibrate these parameters. This could be explained that only in these locations, the complete range of data points from uncongested conditions to congested conditions was available. In this study, the bottleneck locations on the I-95

northbound segment in Miami (NW $79^{\text {th }}$ St and NW $103^{\text {rd }}$ St on-ramp merge area) with different tools and different TFMs were identified for this purpose.

The speeds at the bottleneck locations on the I-95 northbound segment in Miami at NW 79th St and NW 103rd St on-ramp merge area with different tools and different TFMs are compared to the real-world measurements in Figure 4-7 and Figure 4-8. Note 
that the calibrated capacity and jam density in Cube Avenue are 1,850 vehicles per hour per lane (veh/hr/ln) and 190 vehicles per mile per lane (veh/mi/ln), respectively. However, the default values (uncalibrated) for capacity and jam density in both VISUM and DTALite are 1,800 (veh/hr/ln) and $180(\mathrm{veh} / \mathrm{mi} / \mathrm{ln})$, respectively. As shown in Figure 4-7, the average speeds obtained from the implementation of BPR and Akcelik curves in Cube Avenue are close to the observed real-world speed measurements, especially during the time period between 5:00 pm and 6:30 pm. It can be seen that without the application of the calibrated capacity and jam density in DTALite software, the bottleneck location speed does not follow the same trend as the real-world speed, but with the calibrated parameters, the DTALite speeds show the correct trend. The results of VISUM shown in Figure 4-8 indicate that the differences between the runs with the calibrated and uncalibrated capacities are not as high. This could be due to the fact that the default parameters are not significantly different from the calibrated parameters. The comparison of the speeds at the NW 103rd St. bottleneck location in Figure 4-8 shows that the Cube Avenue results overestimated the congestion between 3:30 pm and 5:00 pm. During this period, DTALite and VISUM speed results follow the trend of the real-world measurements better and the use of the calibrated capacity and jam density in the TFM model improves the simulation results. This trend confirms that the queuing model of Cube Avenue underestimates the speed at the point of congestion (closest detector to the bottleneck's starting point). 


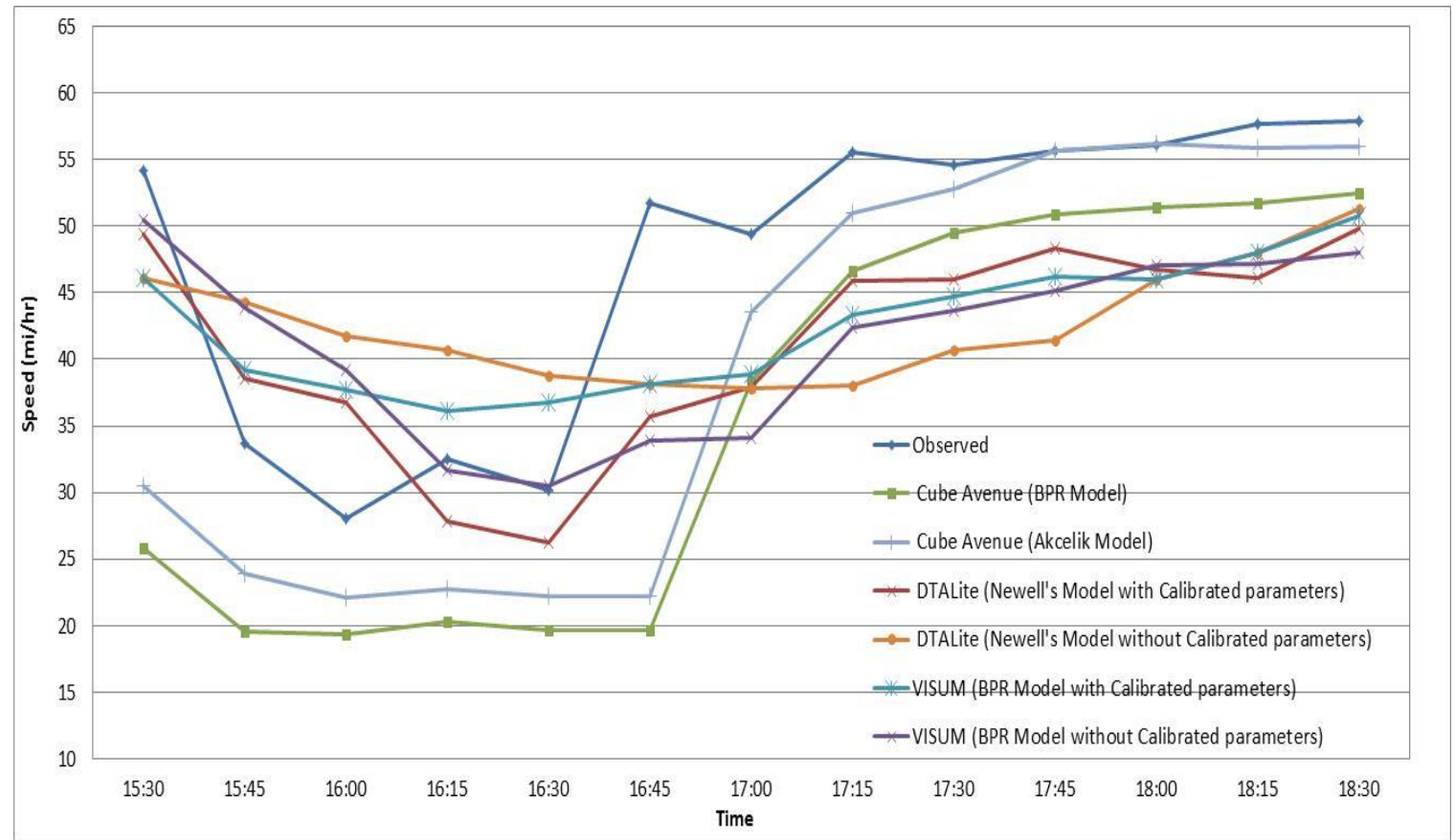

Figure 4-7 Comparison of Different TFMs on Bottleneck Location Speed, NW $79^{\text {th }}$ St

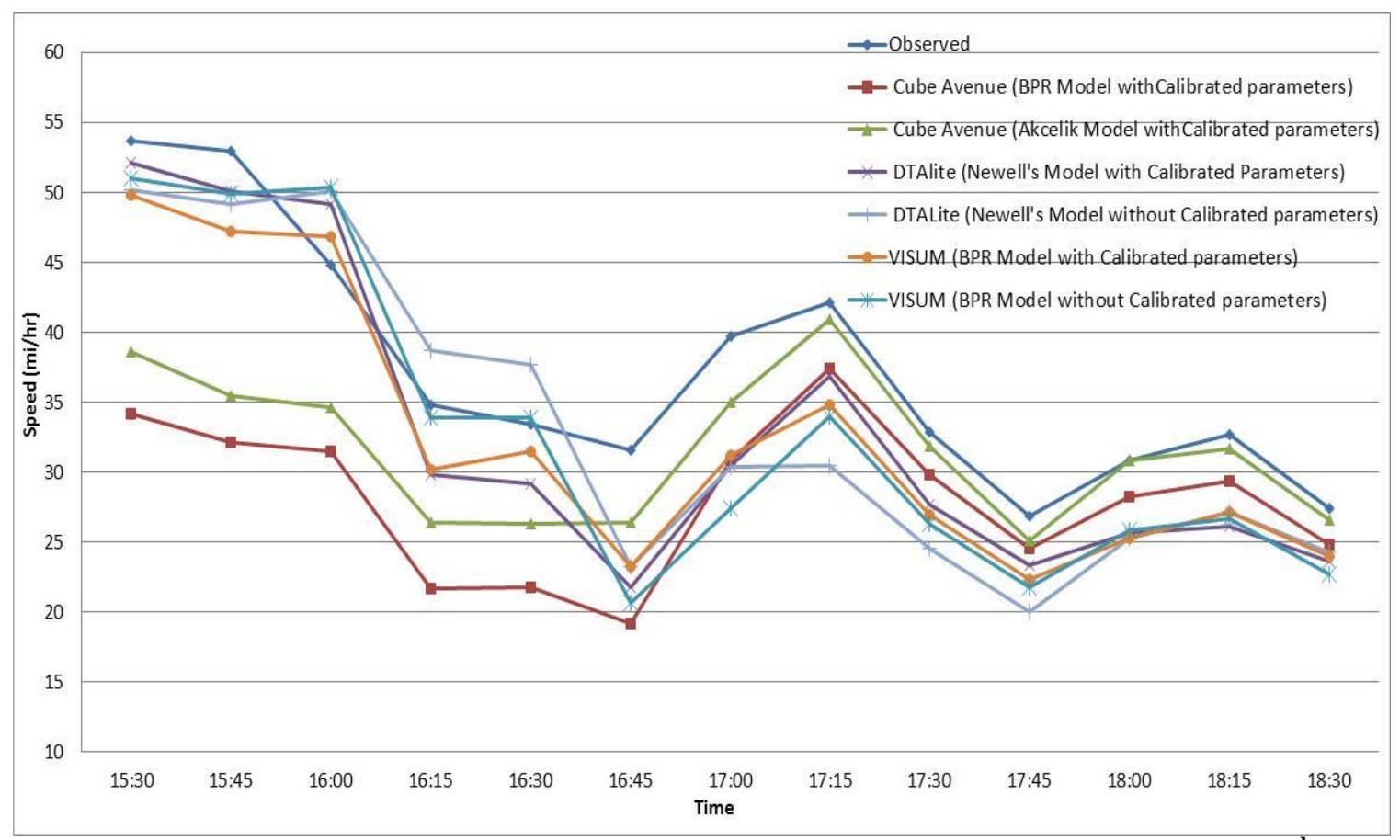

Figure 4-8 Comparison of Different TFMs on Bottleneck Location Speed, NW $103^{\text {rd }} \mathrm{St}$ 
The quality of the supply calibration was evaluated based on the measures of the RMSE and the MPAE. Table 4-2 presents the results of RMSE, MAPE, minimum negative difference, maximum positive difference, and the average difference for the simulated speeds using different DTA tools with and without the calibrated capacity and jam density in the TFM model. Note that the real-world speed measurements were used as a reference. As shown in Table 4-2, the use of the calibrated TFM parameters reduces the deviations from the real-world speeds. Both DTALite and VISUM tools produce better results than Cube Avenue. Using the Ackcelik function with Cube Avenue improved the results. However, in order to assess how each model works, it is not enough to perform the comparison at this point and there is a need to compare the performance along highway segments that capture the spatial extents of congestion.

This is addressed next by speed contours for the studied segment. Figure 4-9 shows the speed contours of the study area utilizing different TFMs in the different DTA tools. As shown in this figure, DTALite and VISUM tools slightly produce better results than Cube Avenue utilizing calibrated parameters along the studied segment. Queue forming and dissipating in DTALite with calibrated parameters are as the real-world. However, using Cube Avenue with BPR function cannot capture the spatial extents of congestion along the studied corridor. 
Table 4-2 Performance Measures for TFM Calibration Results Using Different DTA Tools

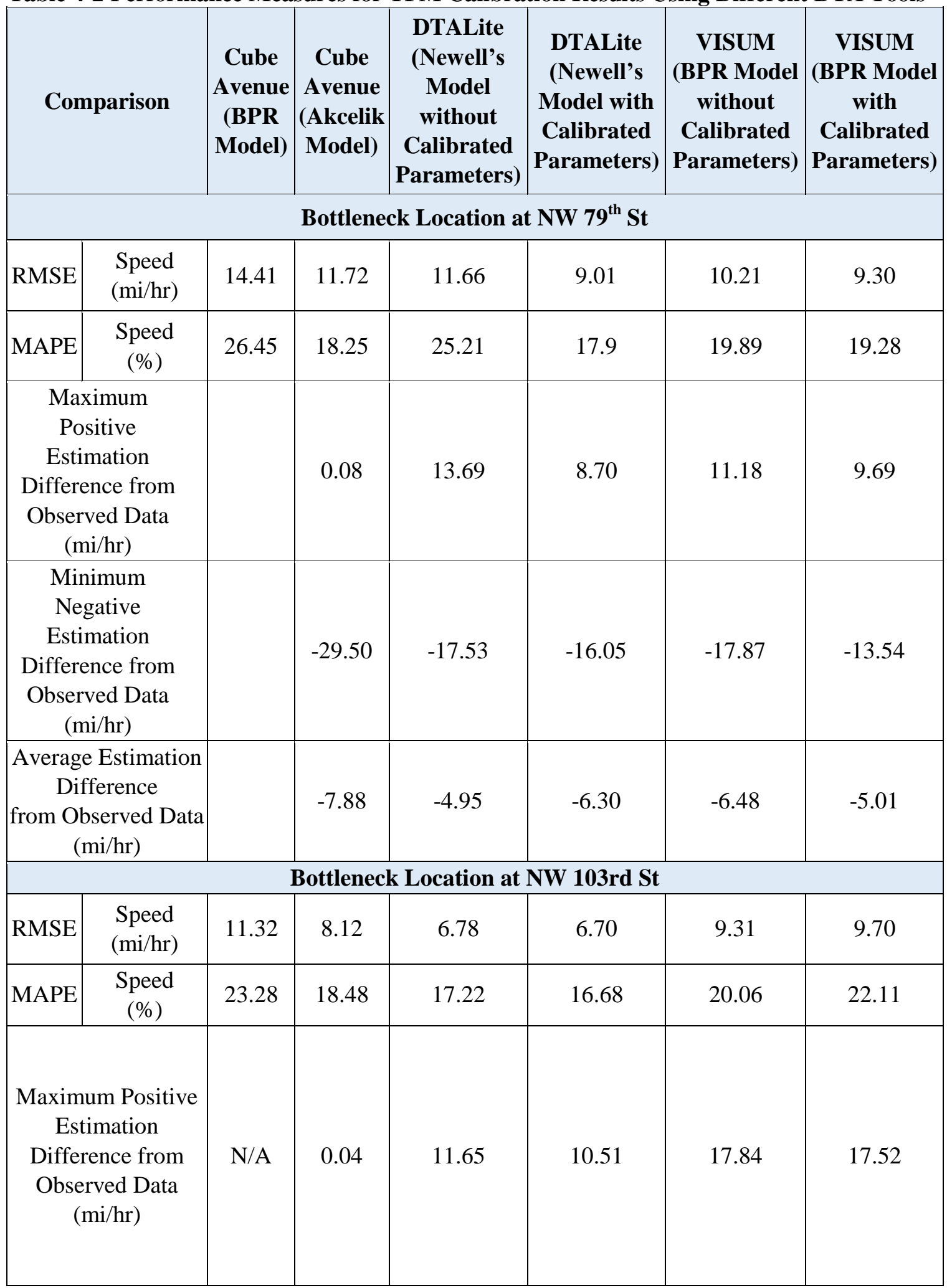




\begin{tabular}{|c|c|c|c|c|c|c|c|}
\hline \multicolumn{2}{|c|}{ Comparison } & $\begin{array}{c}\text { Cube } \\
\text { Avenue } \\
\text { (BPR } \\
\text { Model) }\end{array}$ & $\begin{array}{c}\text { Cube } \\
\text { Avenue } \\
\text { (Akcelik } \\
\text { Model) }\end{array}$ & \begin{tabular}{|c|} 
DTALite \\
(Newell's \\
Model \\
without \\
Calibrated \\
Parameters)
\end{tabular} & $\begin{array}{c}\text { DTALite } \\
\text { (Newell's } \\
\text { Model with } \\
\text { Calibrated } \\
\text { Parameters) }\end{array}$ & $\begin{array}{c}\text { VISUM } \\
\text { (BPR Model } \\
\text { without } \\
\text { Calibrated } \\
\text { Parameters) }\end{array}$ & $\begin{array}{c}\text { VISUM } \\
\text { (BPR Model } \\
\text { with } \\
\text { Calibrated } \\
\text { Parameters) }\end{array}$ \\
\hline \multicolumn{2}{|c|}{$\begin{array}{l}\text { Minimum } \\
\text { Negative } \\
\text { Estimation } \\
\text { Difference from } \\
\text { Observed Data } \\
\quad(\mathrm{mi} / \mathrm{hr})\end{array}$} & -20.87 & -17.54 & -9.79 & -9.78 & -12.30 & -14.07 \\
\hline \multicolumn{2}{|c|}{$\begin{array}{l}\text { Average Estimation } \\
\text { Difference } \\
\text { from Observed Data } \\
(\mathrm{mi} / \mathrm{hr})\end{array}$} & -9.37 & -5.96 & -3.68 & -3.96 & -2.42 & -2.06 \\
\hline \multicolumn{8}{|c|}{ Along Study Segment } \\
\hline RMSE & $\begin{array}{l}\text { Speed } \\
(\mathrm{mi} / \mathrm{hr})\end{array}$ & 26.32 & 19.84 & 21.35 & 13.24 & 20.08 & 14.18 \\
\hline MAPE & $\begin{array}{c}\text { Speed } \\
(\%)\end{array}$ & 44.12 & 29.77 & 38.28 & 21.68 & 36.80 & 23.65 \\
\hline \multicolumn{2}{|c|}{$\begin{array}{l}\text { Maximum Positive } \\
\text { Estimation } \\
\text { Difference from } \\
\text { Observed Data } \\
(\mathrm{mi} / \mathrm{hr})\end{array}$} & 3.24 & 2.98 & 5.68 & 4.25 & 5.16 & 5.46 \\
\hline \multicolumn{2}{|c|}{$\begin{array}{l}\text { Minimum } \\
\text { Negative } \\
\text { Estimation } \\
\text { Difference from } \\
\text { Observed Data } \\
\text { (mi/hr) }\end{array}$} & -12.49 & -10.18 & -8.45 & -7.30 & -9.78 & -10.14 \\
\hline \multicolumn{2}{|c|}{$\begin{array}{l}\text { Average Estimation } \\
\text { Difference } \\
\text { from Observed Data } \\
(\mathrm{mi} / \mathrm{hr})\end{array}$} & -8.48 & -4.89 & -6.45 & -3.17 & -5.38 & -3.36 \\
\hline
\end{tabular}



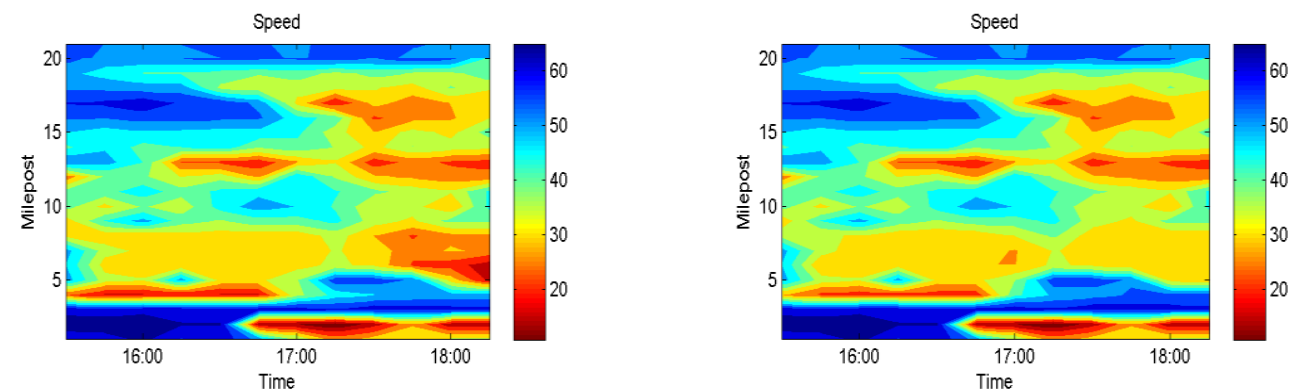

Cube Avenue (BPR Model with Calibrated Parameters) Cube Avenue (Akcelik Model with Calibrated Parameters)
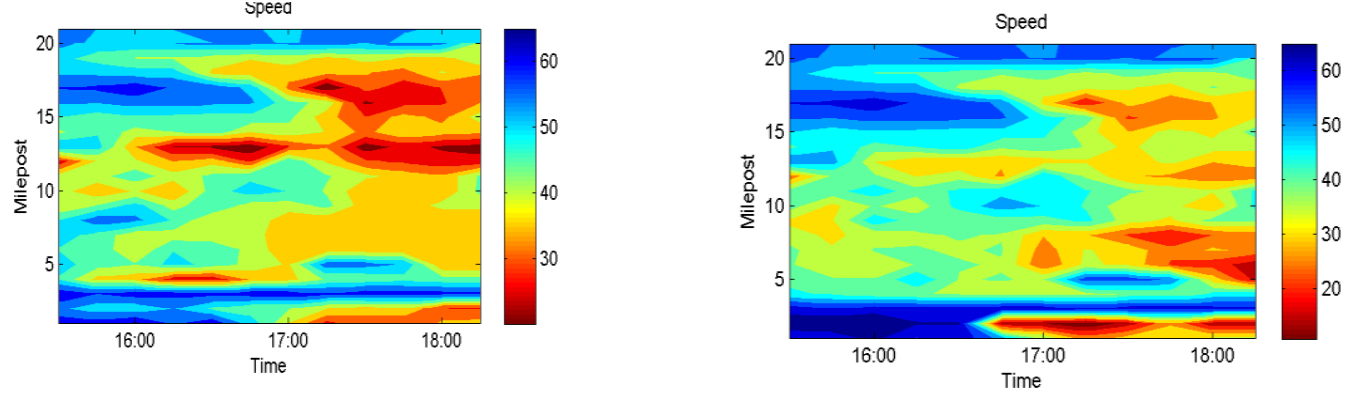

DTAlite (Newell's Model with Calibrated Parameters)

DTAlite (Newell's Model without Calibrated Parameters)
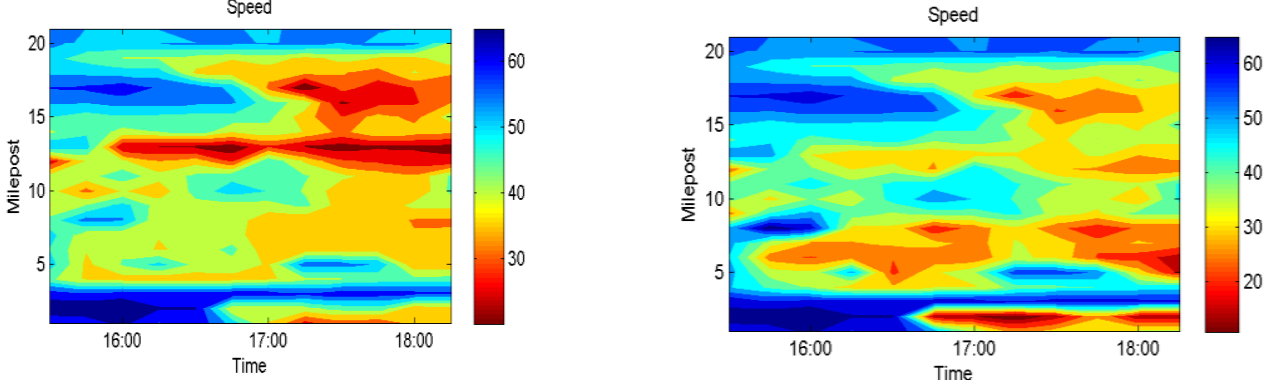

VISUM (BPR Model with Calibrated Parameters)

VISUM (BPR Model without Calibrated Parameters)

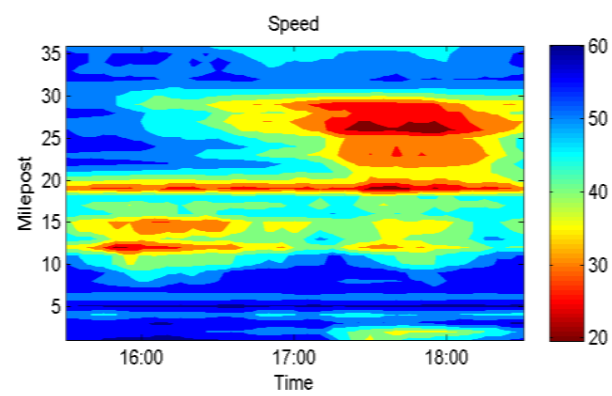

Observed

Figure 4-9 Speed Contours for the Study Corridor Utilizing Different TFMs 


\subsection{Origin-Destination Matrix Estimation (ODME)}

The estimation of time-variant trip matrices is an important step in dynamic traffic assignment-based tools. DTA analysis requires dynamic or time-variant trip matrices specified for short time intervals (e.g., 15 minutes or 30 minutes). However, regional demand models are usually daily or time-of-day models that can only produce daily trip matrix or trip matrix for peak periods. An origin-destination matrix estimation process is needed to fill in such a gap, that is, to estimate the trip tables for short intervals based on an initial matrix obtained from the regional demand model and field data. A simple ODME method is the factorization method that applies factors to convert daily or timeof-day demand matrices to matrices for short time intervals. The more widely used methods are estimating the O-D matrix by minimizing the difference between the simulated performance measures and real-world measurements using an optimization procedure. The resulting time-variant matrices, when loaded onto the calibrated network, should be able to replicate the observed link volumes and congestion patterns.

An ODME tool is usually provided with currently available static and dynamic traffic assignment software. For example, Cube Analyst and its updated version Analyst Drive are the ODEM modules in Cube. The TflowFuzz module is the ODME module in VISUM. An ODME tool can also be accessed in the NEXTA interface of DTALite. As stated earlier, the time-variant trip matrix for the study network has been calibrated by Hadi et al. (2013). The core of that demand calibration in that study was the application of a static assignment-based ODME and further fine-tuning the resulting matrix to improve the results. The exploration of a further refinement of the O-D matrix using 
dynamic O-D estimation was done to see the improvement of the assignment results in present research. The following were tested in this study regarding the ODME process.

- How well do the assignment tools work when the O-D matrix is estimated using the ODME procedure that is included and interfaced with another tool?

- Whether utilizing the ODME procedure of tools can improve on the O-D estimated by an ODME procedure from another assignment tool.

The sensor data include 15-minutes volume counts collected at 87 locations on the GPL, ML, and ramps of the I-95 facility in Miami, FL, and were read by the ODME module in each tool. Figure 4-10 displays the green squares to represent the detector.

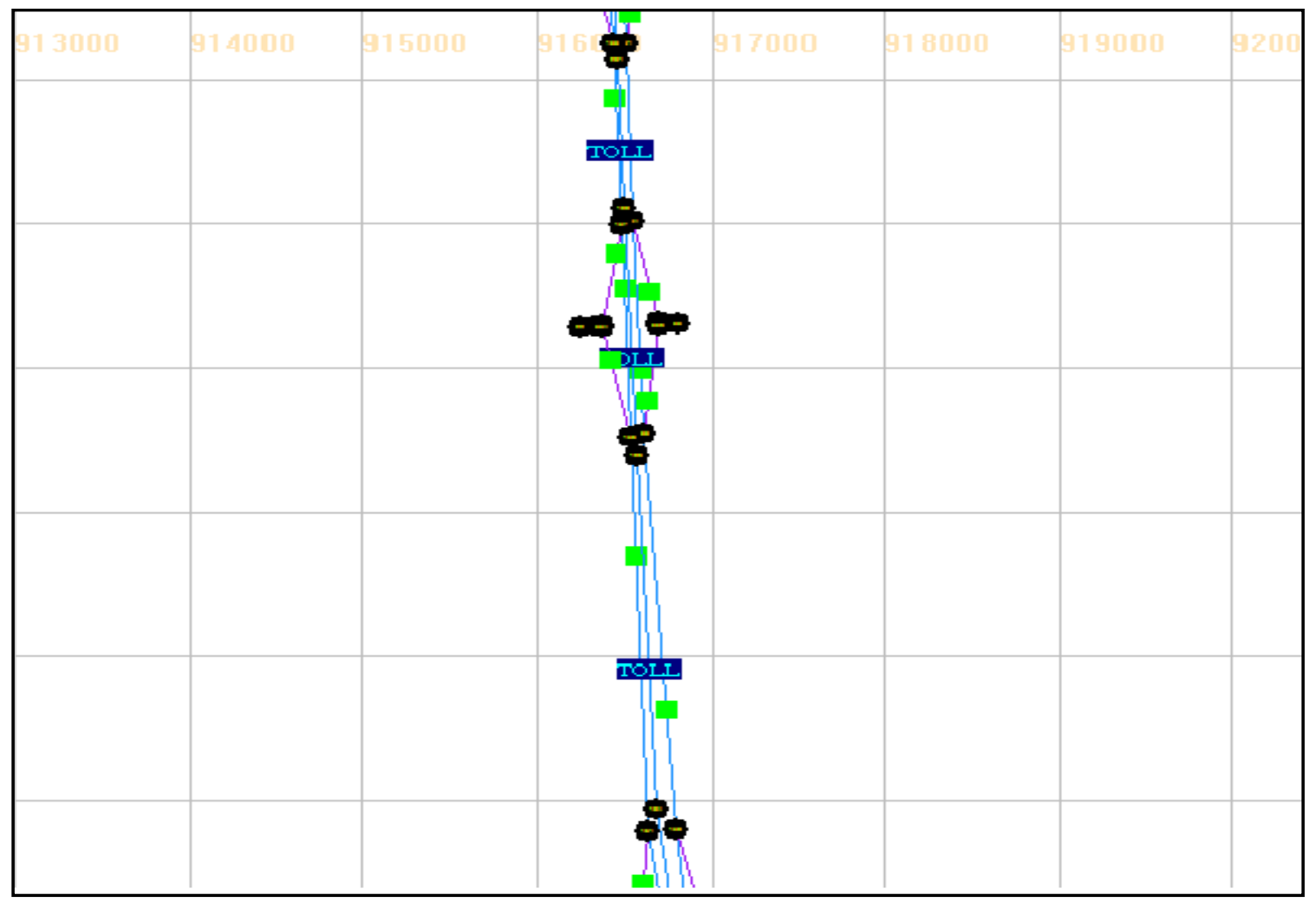

Figure 4-10 Schematic of Sensor Locations for the ODME Process 


\subsubsection{ODME Process in Cube}

Figure 4-11 presents a comparison of simulated link volumes with real-world traffic counts for two different types of matrices. The first type represents the factorized demand matrix based on the matrix extracted from regional demand. The second is the calibrated demand matrix from the study of Hadi et al. (2013), which was obtained by using a combination of the ODME procedure and fine-tuning of the results. Note that these simulated results are obtained from running the Cube Avenue dynamic traffic assignment tool. As shown in this figure, the simulated link volumes cannot replicate the observed link volumes when using the factorized initial demand matrix obtained based on the regional demand model matrix. The corresponding $\mathrm{R}^{2}$ is only 0.29 . With the calibrated demand, the simulated link volumes become more similar to the observed link volumes with a $\mathrm{R}^{2}$ value of 0.80 .
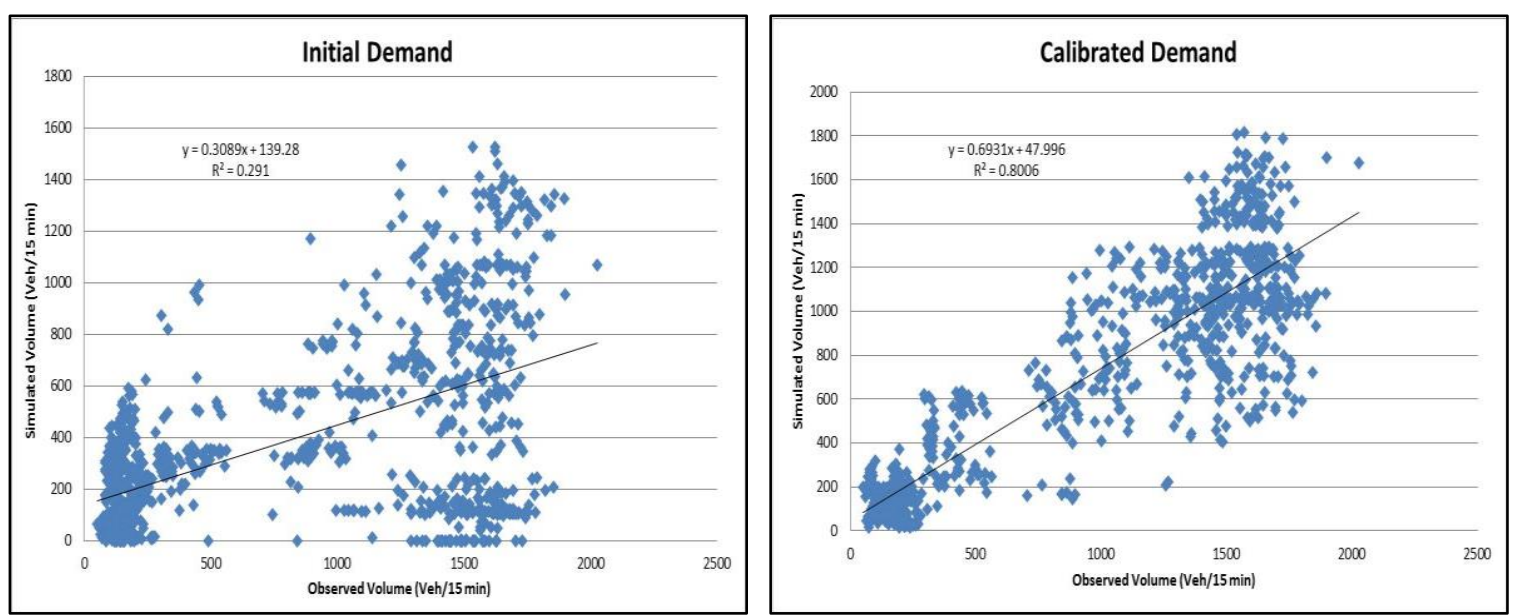

Figure 4-11 Comparison of Observed vs. Simulated Link Volume Produced by Cube Avenue Utilizing DTA

Figure 4-12 presents the comparison results when using the ODME procedure in Cube. When using the initial factorized input demand matrix as an input to the ODME, the $\mathrm{R}^{2}$ value only improved from 0.29 to 0.39 . This indicates that inputting low quality 
demand matrices to the ODME process does not allow the ODME to provide good results. When using the calibrated demand as the input for the ODME process, the improvement in the $\mathrm{R}^{2}$ value is not significant from 0.29 to 0.39 and from 0.8 to 0.81 for the initial demand and calibrated demand, respectively. This is due to the fact that the ODME in Cube has already been used as part of the derivation of the calibration matrix in the previous study. In addition, the Cube ODME process utilizes demands from the static assignment during the optimization process and is not able to capture queue spillback in space and time.
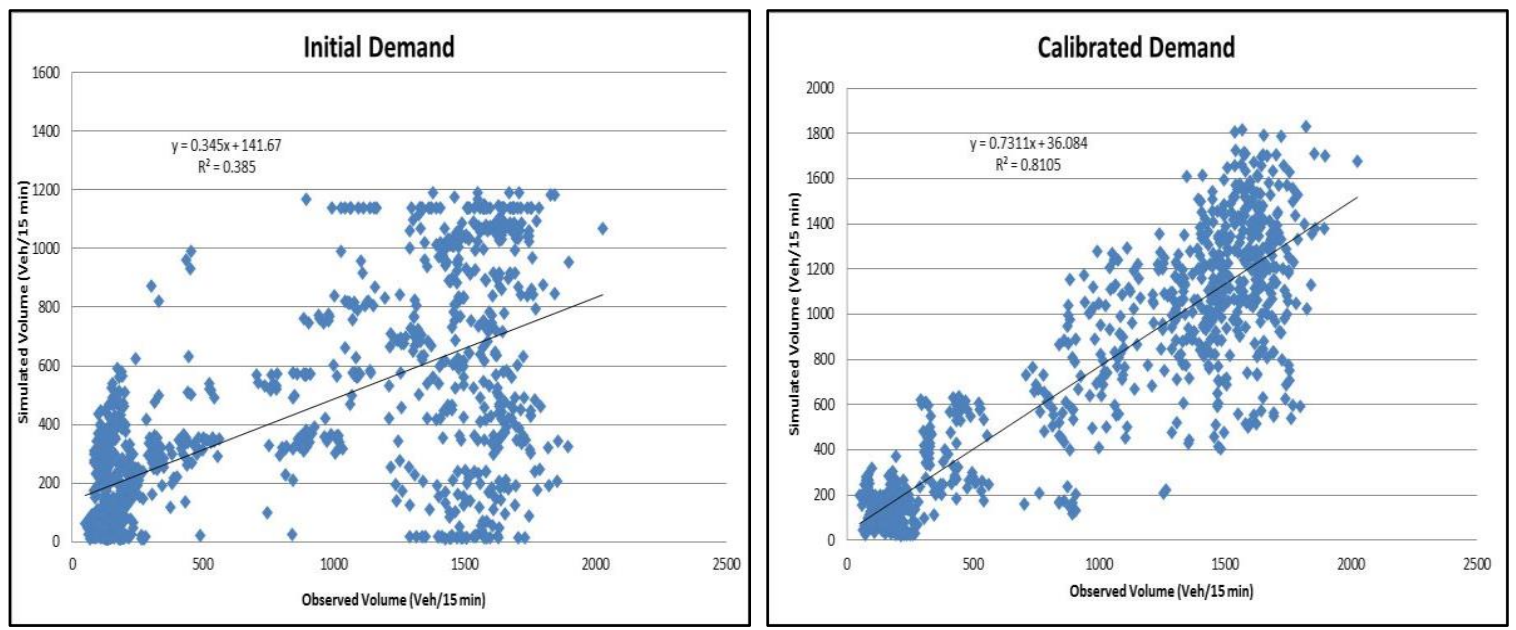

Figure 4-12 Comparison of Simulated vs. Observed Link Volumes after Running ODME in Cube

\subsubsection{ODME Process in VISUM}

A matrix estimation function is provided in VISUM through a dynamic TFlowFuzzy (TFF) module. As with the ODME modules available in other tools, it iteratively adjusts the demand matrix such that the assigned link volume can be close to reference data such as count data. It is a dynamic process that is able to capture queue spillback in space and time. As mentioned earlier in Section 4.2, one of the tasks in this research is to examine how the DTA tool performs when using the calibrated matrices 
from another DTA tool. Therefore, in this study, the 15-minutes factorized and calibrated O-D matrices from the research by Hadi et al., (2013) were used as input matrices for the VISUM analysis. Figure 4-15 presents the dynamic traffic assignment results for link volumes using the factorized demand matrices from the regional model, as well as the calibrated Cube demand matrices in VISUM. As shown in this figure, most of the simulated link volume using the initial factorized demand matrices are either underestimated or overestimated with a $\mathrm{R}^{2}$ value of 0.47 . Also, as shown in this figure, running the VISUM DTA with the calibrated demand can produce link volumes that are relatively close to the observed values with a $\mathrm{R}^{2}$ of 0.82 , which is similar to the Cube Avenue results. Figure 4-15 and Figure 4-16 present the results of simulated link volume after utilizing the ODME in VISUM. Significant improvements in the simulated link volumes can be observed in Figure 4-16. Compared to the results in Figure 4-15, the $\mathrm{R}^{2}$ value improved to 0.79 and 0.96 when using the uncalibrated and calibrated matrices as inputs to the VISUM ODME process, respectively. This indicates that the dynamic ODME procedure used in VISUM is more effective than the static assignment-based ODME in Cube that was implemented, as discussed earlier. 

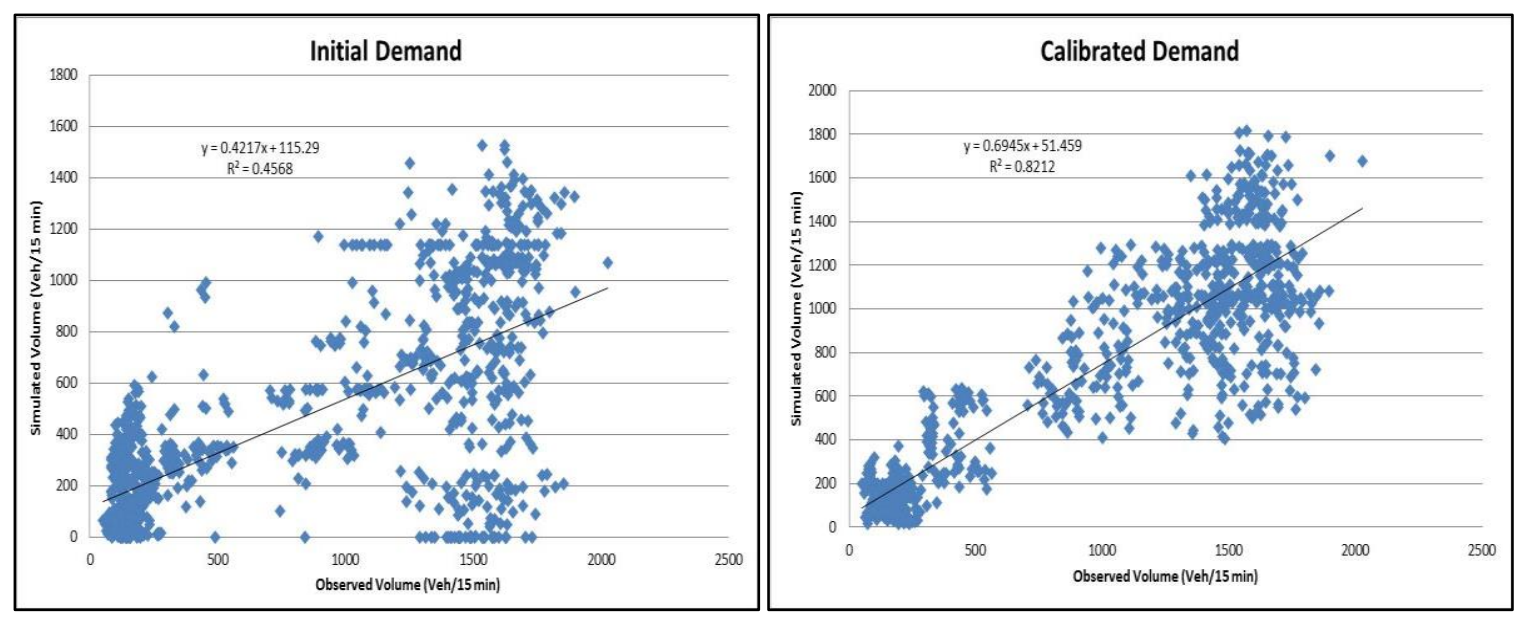

Figure 4-13 Comparison of Observed vs. Simulated Volume Produced by VISUM Utilizing DTA
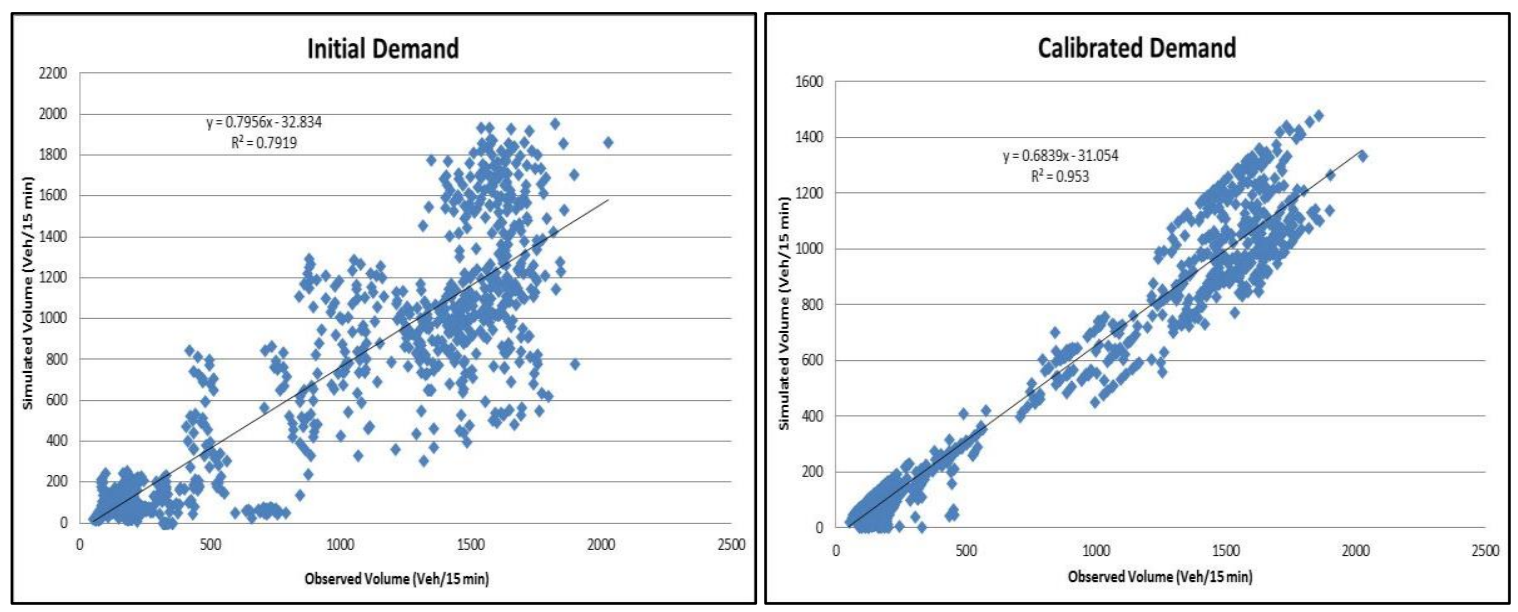

Figure 4-14 Comparison of Simulated Link Volumes vs. Observed Link Volume after Running ODME in VISUM

\subsubsection{ODME Process in DTALite}

In the NEXTA, the graphic user interface of DTALite, the user can run a dynamic ODME process by enabling the ODME mode in the "input_scenario_settings.csv" file and configuring the setups in the "ODME_Settings.txt" file. The ODME parameters, such as the number of iterations, the amount of adjustment allowed per iteration, and the calibration time period; which could be a portion of the modeling period, can be specified in these files. Figure 4-15 and Figure 4-16 compare the simulated link volume with the 
observed values with and without using the dynamic ODME process in DTALite. The results in these two figures are very similar to those obtained using the VISUM software. With the input of the factorized demand matrix based on the regional model, the implementation of ODME can improve the $\mathrm{R}^{2}$ value from 0.47 to 0.81 , while the $\mathrm{R}^{2}$ value can increase from 0.82 to 0.96 , with the calibrated demand matrix as input. Again, these results emphasize the importance of the dynamic ODME compared to the static ODME of Cube.
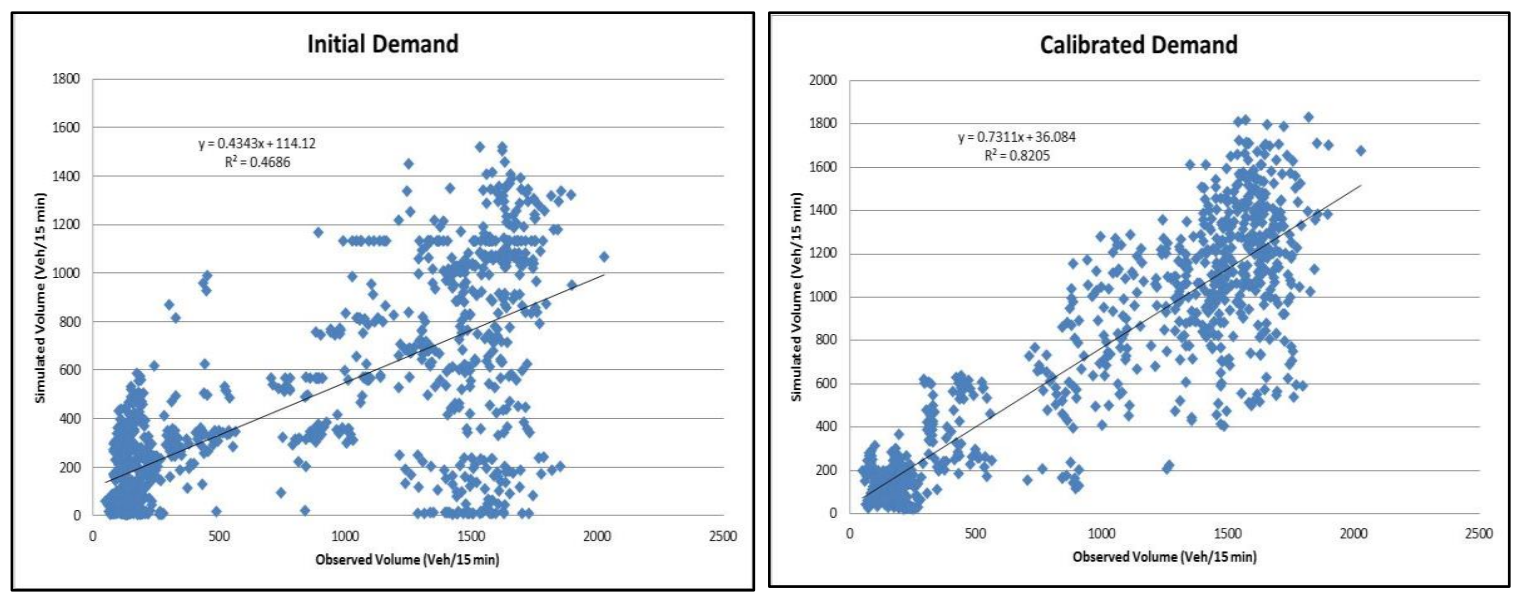

Figure 4-15 Comparison of Observed Link Volumes vs. Simulated Link Volume Produced by DTALite Utilizing DTA
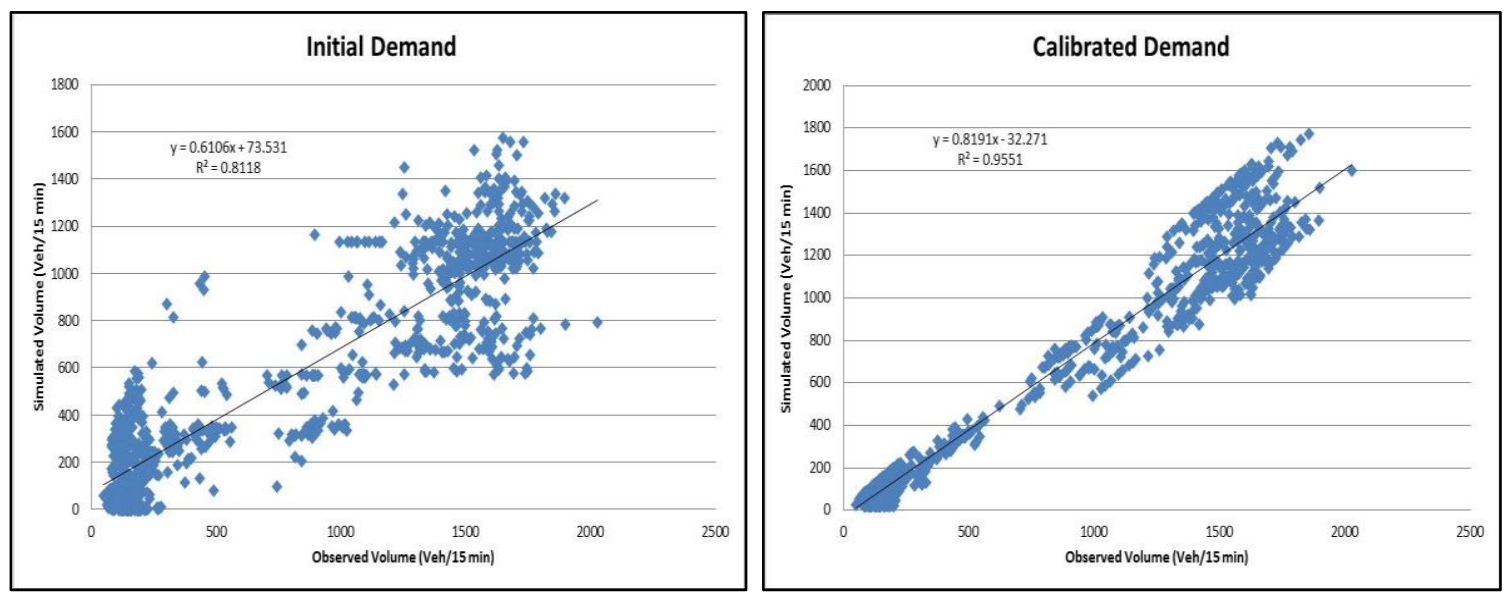

Figure 4-16 Comparison of Simulated Link Volumes vs. Observed Link Volume after Running ODME in DTALite 


\subsubsection{Summary of Tool Assessment for Demand Estimation}

Demand matrix estimation is an undetermined problem as the number of equations for link counts is usually much lower than the number of unknown O-D pairs. It is important, therefore, to manage the estimation process to ensure the reasonableness and the correctness of the estimated demands. Table 4-3 and Table 4-4 compare the goodness-of-fit for the simulated link volume based on the above results. The measures listed in these two tables show that DTALite can produce better volume results than the other two tools, although the VISUM software results are also similar. A better demand matrix used as input to the ODME process (such as the previously calibrated demand matrix) can produce a more realistic replication of real-world volume counts, compared to utilizing a simple factorized demand matrix. The implementation of the dynamic ODME in VISUM and DTALite can better capture the queue forming and dissipation than the static assignment-based ODME implemented in Cube, which can result in more accurate volume counts. As shown inTable 4-3 Table 4-3 and Table 4-4, the ODME module in Cube does not improve the results significantly compared to that based on static assignment. However, the dynamic ODME in VISUM and DTALite are able to produce much better results because they enhance the model for congestion pattern replication than the ODME based on static assignment in Cube.

Table 4-3 Goodness of Fit for Simulated Volume Based on Factorized Demand Matrix

\begin{tabular}{|c|c|c|c|c|c|c|}
\hline \multirow{3}{*}{ Goodness-of-Fit Statistics } & \multicolumn{6}{|c|}{ Initial Demand } \\
\hline & \multicolumn{3}{|c|}{$\begin{array}{c}\text { Running DTA } \\
\text { without ODME }\end{array}$} & \multicolumn{3}{|c|}{ Running ODME Optimization } \\
\hline & Cube & VISUM & DTALite & Cube & VISUM & DTALite \\
\hline MAE & 123.48 & 108.13 & 101.56 & 112.51 & 80.74 & 68.21 \\
\hline MAPE (\%) & 20.61 & 18.69 & 16.11 & 15.35 & 13.32 & 12.52 \\
\hline RMSE(veh/ln/15min) & 181.32 & 158.79 & 149.14 & 165.21 & 118.56 & 100.16 \\
\hline R squared & 0.29 & 0.46 & 0.47 & 0.39 & 0.72 & 0.81 \\
\hline
\end{tabular}




\begin{tabular}{|c|c|c|c|}
\hline $\begin{array}{c}\text { Improved Demand Estimation Utilizing } \\
\text { ODME Optimization (\%) }\end{array}$ & 8.88 & 25.33 & 32.84 \\
\hline
\end{tabular}

Table 4-4 Goodness of Fit for Simulated Volume Based on Calibrated Demand Matrix

\begin{tabular}{|c|c|c|c|c|c|c|}
\hline \multirow{3}{*}{ Goodness-of-Fit Statistics } & \multicolumn{6}{|c|}{ Calibrated Demand } \\
\hline & \multicolumn{3}{|c|}{$\begin{array}{c}\text { Running DTA } \\
\text { without ODME }\end{array}$} & \multicolumn{3}{|c|}{ Running ODME Optimization } \\
\hline & Cube & VISUM & DTALite & Cube & VISUM & DTALite \\
\hline MAE & 67.79 & 62.13 & 62.19 & 63.44 & 59.71 & 55.18 \\
\hline MAPE (\%) & 11.21 & 11.09 & 10.98 & 11.02 & 10.9 & 10.62 \\
\hline RMSE(veh/ln/15min) & 95.15 & 91.24 & 91.33 & 93.15 & 87.68 & 81.03 \\
\hline R squared & 0.80 & 0.821 & 0.832 & 0.81 & 0.95 & 0.96 \\
\hline \multicolumn{4}{|c|}{$\begin{array}{c}\text { Improved Demand Estimation Utilizing } \\
\text { ODME Optimization }(\%)\end{array}$} & 2.09 & 3.9 & 11.27 \\
\hline
\end{tabular}

\subsection{Application of MRM to ML}

The developed MRM framework is applied in this section to model the ML and the impacts of associated operational and pricing strategies. The ML are simulated using the different levels of modeling as described earlier in this research. The resulting demand and performance measures of the ML, GPL, and alternative routes utilizing different modeling strategies were examined and compared with each other, as well as to real-world traffic and toll data. The sensitivity analysis of the value of time distribution, value of reliability, and toll pricing policies were also conducted in this research.

\subsubsection{VOT Consideration in ML Modeling}

As explained earlier, VOT converts the monetary value of toll cost into equivalent time. This equivalent time can be then added to the utility function of the ML facility, based on Equation 3-5. In most DTA tools and their applications, an average value of VOT is commonly used. However, a distribution of VOT, possibly combined with the categorization of users by income and/or other measures, can better capture the preference of different road users In the SHRP 2 C04 project, a lognormal distribution 
was recommended for the distribution of the value of time and the default average VOT used in the SHRP 2 C04 was, \$20 per hour, as explained in detail in Section 3.2.1.

In this study, using the fixed VOT and distribution of VOT was tested utilizing the DTALite because this tool allows the users to define a distribution for the VOT, which is not possibly or easily done in the other tools tested in this study. The default average VOT in DTALite is $\$ 1$ for every 5 minutes (that is, $\$ 12 /$ hour). Figure 4-17 shows the default distributions for VOT based in the DTALite.

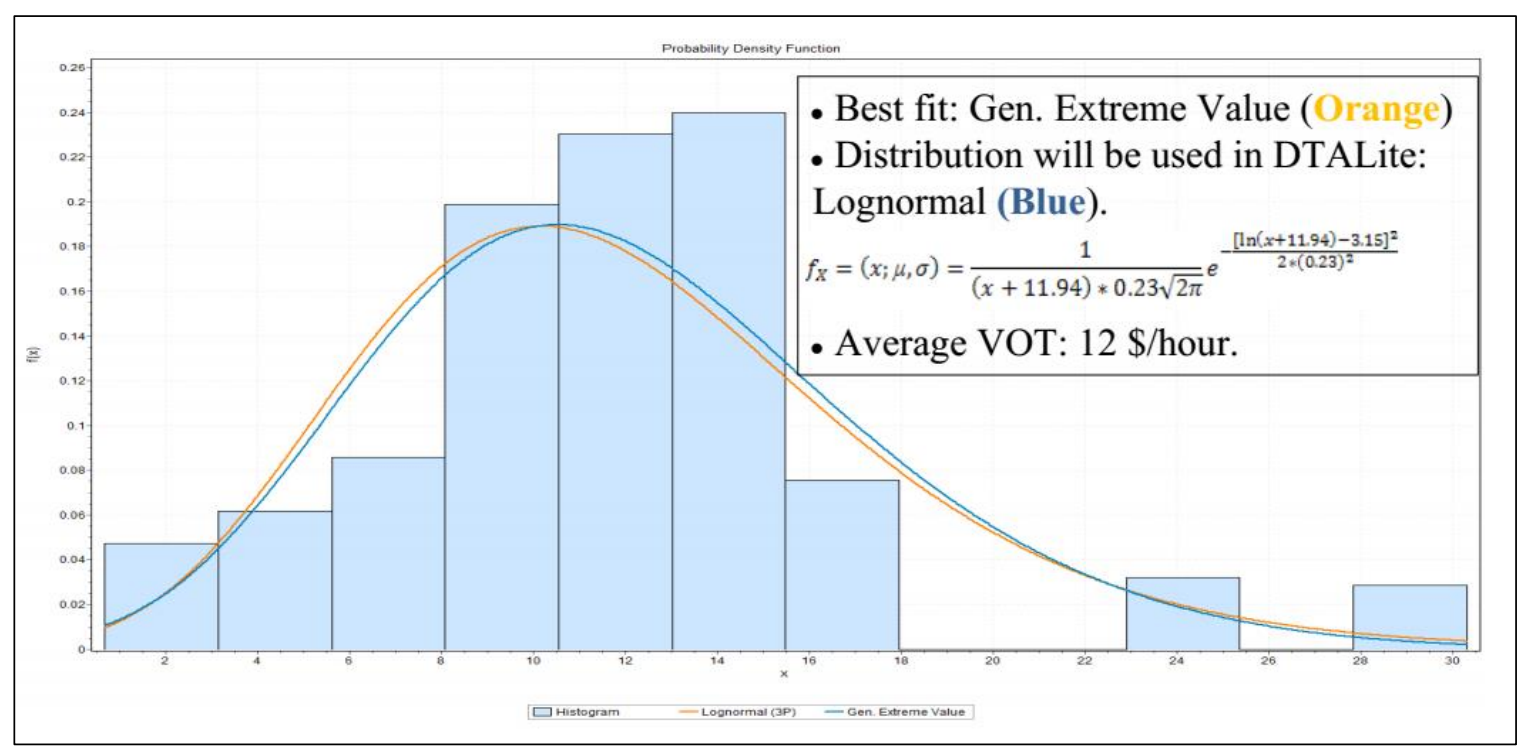

Figure 4-17 VOT Distribution in DTALite (DTALite User Manual, 2012)

In this study, the toll data for I-95 northbound in April, 2015 was also obtained from FDOT D6 TMC. Averaging the toll values for over a period of 10 weekdays shows that the average toll is between $\$ 6$ and $\$ 7$ during the congested PM peak period with an average value of $\$ 6.30$, as shown in Table $4-5$. The time saved by travelers based on realworld detector data for non-incident days is usually between 6 to 10 minutes depending on the congestion level in the GPL. Paying an average toll value of \$6.30 implies that the traveler's value of time is about $\$ 27$ to $\$ 43$. 
Table 4-5 Real-World Toll Value (\$) for I-95 Northbound in April, 2015

\begin{tabular}{|c|c|c|c|c|c|c|c|c|c|c|c|}
\hline Time & Day 1 & Day 2 & Day 3 & Day 4 & Day 5 & Day 6 & Day 7 & Day 8 & Day 9 & Day 10 & Average \\
\hline $\mathbf{1 5 : 3 1}$ & 5.00 & 5.50 & 5.50 & 5.50 & 5.75 & 5.75 & 5.75 & 5.75 & 6.00 & 5.75 & 5.63 \\
\hline $\mathbf{1 5 : 4 2}$ & 6.00 & 6.50 & 6.00 & 6.25 & 5.50 & 5.75 & 6.00 & 6.75 & 6.50 & 6.25 & 6.15 \\
\hline $\mathbf{1 6 : 1 0}$ & 5.50 & 7.25 & 5.75 & 5.75 & 5.75 & 6.00 & 6.00 & 6.00 & 6.00 & 6.00 & 6.00 \\
\hline $\mathbf{1 6 : 2 4}$ & 5.50 & 7.25 & 5.75 & 5.75 & 6.00 & 6.00 & 6.25 & 6.00 & 6.00 & 6.00 & 6.05 \\
\hline $\mathbf{1 6 : 3 6}$ & 5.50 & 7.00 & 5.75 & 5.75 & 6.00 & 6.00 & 6.25 & 6.25 & 6.25 & 6.00 & 6.07 \\
\hline $\mathbf{1 6 : 5 1}$ & 5.50 & 7.00 & 5.75 & 5.75 & 7.00 & 6.00 & 6.25 & 7.25 & 6.50 & 6.00 & 6.30 \\
\hline $\mathbf{1 7 : 1 0}$ & 5.50 & 7.00 & 6.00 & 6.50 & 7.00 & 6.25 & 6.25 & 6.75 & 6.75 & 6.25 & 6.42 \\
\hline $\mathbf{1 7 : 2 4}$ & 5.75 & 7.50 & 6.00 & 7.00 & 7.50 & 7.25 & 6.5 & 6.75 & 7.00 & 7.00 & 6.82 \\
\hline $\mathbf{1 7 : 3 6}$ & 5.75 & 6.50 & 5.50 & 7.00 & 7.75 & 7.00 & 7.00 & 7.25 & 7.50 & 7.00 & 6.82 \\
\hline $\mathbf{1 7 : 4 1}$ & 5.75 & 7.75 & 5.50 & 7.50 & 7.50 & 6.75 & 6.75 & 7.25 & 7.00 & 6.75 & 6.85 \\
\hline $\mathbf{1 8 : 1 0}$ & 5.50 & 7.75 & 5.50 & 6.75 & 6.75 & 6.00 & 6.75 & 7.00 & 6.5 & 6.00 & 6.45 \\
\hline $\mathbf{1 8 : 2 1}$ & 5.50 & 7.00 & 6.25 & 6.25 & 6.25 & 5.50 & 6.25 & 6.25 & 6.5 & 6.00 & 6.17 \\
\hline
\end{tabular}

Based on the above discussion, a sensitivity analysis was conducted in this study to find the best value of time that produces the observed shift to the ML in the DTALite tool. A number of average VOT values, $\$ 12, \$ 20, \$ 30, \$ 40$, and $\$ 50$, were used in the sensitivity analysis (utilizing a distribution for VOT) and the results of diverted volume to ML are presented in Figure 4-18 and Table 4-6. From this figure and table, it appears that the value of time of $\$ 40$ produces the closest results to the real-world diverted volume to ML, which is much greater than the value of $\$ 13.44$ used in the SERPM model. It should be noted that in addition to saved travel time, this VOT most likely accounts for other factors not considered in the generalized cost function such as travel time reliability, comfort, safety, and the travel time experience in past days, which could include more congested days and incident days compared to the present day. 


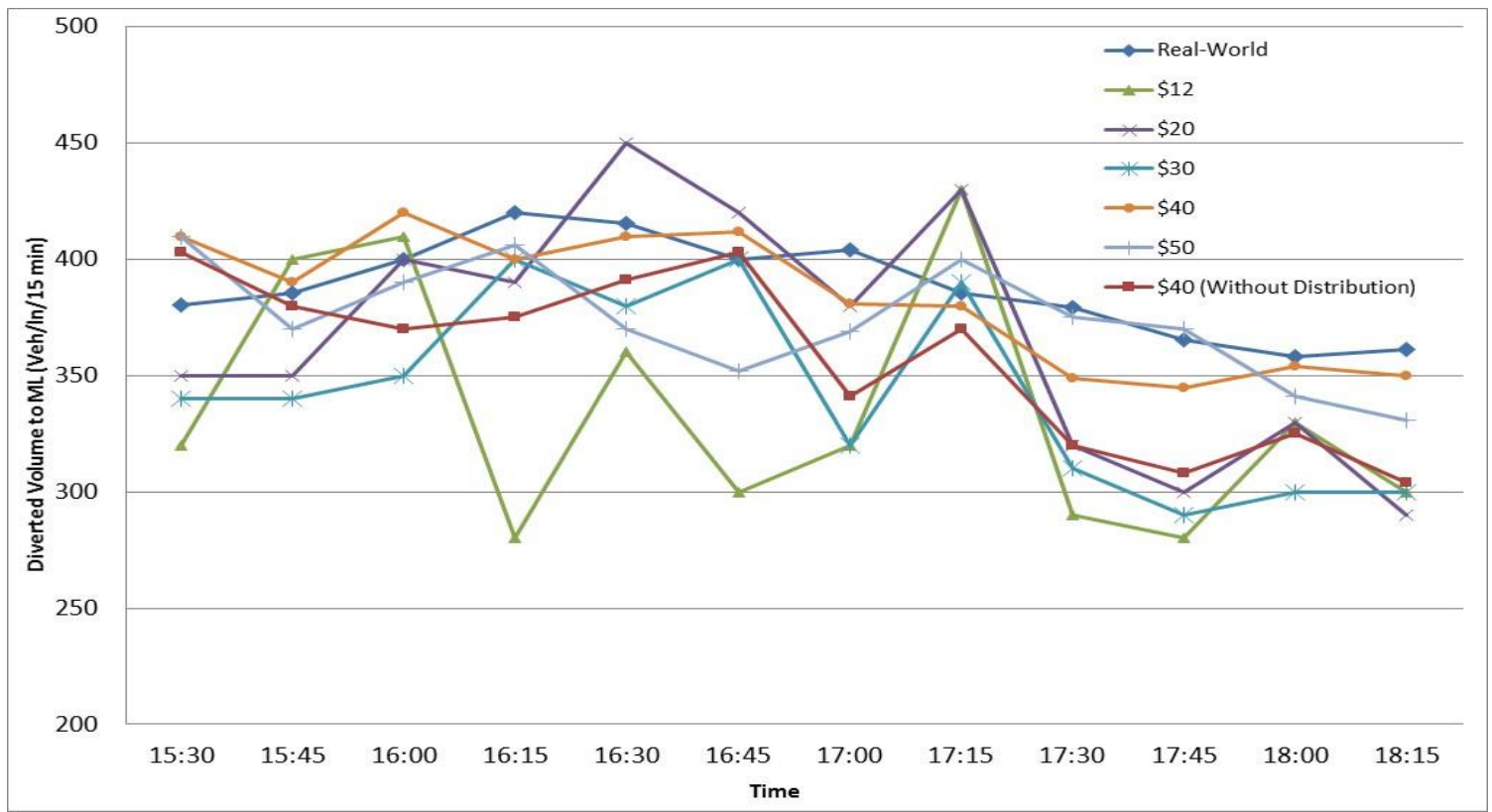

Figure 4-18 Comparison of Diverted Volume to ML for Different VOTs

The next step was to examine if utilizing a distribution of VOT, instead of a fixed value produces better correspondence to real-world diversion. A fixed value of time of $\$ 40$ (without utilizing distribution VOT) was used to estimate the diverted volume to ML to compare the results with using the same average VOT from a distribution.

Table 4-6 Diverted Volumes (veh//n/15min) to ML for Different VOTs

\begin{tabular}{|c|c|c|c|c|c|c|c|}
\hline $\begin{array}{c}\text { Time } \\
\mathbf{( P M )}\end{array}$ & $\mathbf{\$ 1 2}$ & $\mathbf{\$ 2 0}$ & $\mathbf{\$ 3 0}$ & $\mathbf{\$ 4 0}$ & $\mathbf{\$ 5 0}$ & $\begin{array}{c}\mathbf{\$ 4 0} \text { Fixed } \\
\text { (without Distribution) }\end{array}$ & Real-World \\
\hline $\mathbf{1 5 : 3 0}$ & 320 & 350 & 340 & 410 & 410 & 403 & 380 \\
\hline $\mathbf{1 5 : 4 5}$ & 400 & 350 & 340 & 390 & 370 & 380 & 385 \\
\hline $\mathbf{1 6 : 0 0}$ & 410 & 400 & 350 & 420 & 390 & 370 & 400 \\
\hline $\mathbf{1 6 : 1 5}$ & 280 & 390 & 400 & 400 & 406 & 375 & 420 \\
\hline $\mathbf{1 6 : 3 0}$ & 360 & 450 & 380 & 410 & 370 & 391 & 415 \\
\hline $\mathbf{1 6 : 4 5}$ & 300 & 420 & 400 & 412 & 352 & 403 & 400 \\
\hline $\mathbf{1 7 : 0 0}$ & 320 & 380 & 320 & 381 & 369 & 341 & 404 \\
\hline $\mathbf{1 7 : 1 5}$ & 430 & 430 & 390 & 380 & 400 & 370 & 385 \\
\hline $\mathbf{1 7 : 3 0}$ & 290 & 320 & 310 & 349 & 375 & 320 & 379 \\
\hline $\mathbf{1 7 : 4 5}$ & 280 & 300 & 290 & 345 & 370 & 308 & 365 \\
\hline $\mathbf{1 8 : 0 0}$ & 330 & 330 & 300 & 354 & 341 & 325 & 358 \\
\hline $\mathbf{1 8 : 1 5}$ & 300 & 290 & 300 & 350 & 331 & 304 & 361 \\
\hline
\end{tabular}


The For each value of VOT, the differences between the simulated diverted volumes and the real-world observations were quantified in terms of RMSE and the MAPE, as listed in Table 4-7. The MAPE and RMSE values in Table 4-7 confirm that the estimated distribution of VOT with an average VOT of $\$ 40$ produces better results compared to the real-world volumes on the ML. As indicated in this table, the corresponding RMSE and MAPE for this case are $18 \mathrm{veh} / \mathrm{ln} / 15 \mathrm{~min}$ and $4.01 \%$, respectively. The results in Table 4-7 also confirm that the use of fixed VOT without utilizing the distribution of VOT does not produces as good results as when using a VOT distribution. The RMSE and MAPE values for the fixed $\$ 40$ VOT are $40 \mathrm{veh} / \mathrm{ln} / 15 \mathrm{~min}$ and $9.03 \%$, respectively.

Table 4-7 Goodness of Fit Statistics for Diverted Volume Replication Based on Different VOT

\begin{tabular}{|c|c|c|c|c|c|c|}
\hline & \multicolumn{6}{|c|}{ Value of Time \$ (VOT) } \\
\cline { 2 - 7 } Goodness-of-Fit Statistics & $\mathbf{\$ 1 2}$ & $\mathbf{\$ 2 0}$ & $\mathbf{\$ 3 0}$ & $\mathbf{\$ 4 0}$ & $\mathbf{\$ 5 0}$ & $\begin{array}{c}\mathbf{\$ 4 0 \text { Fixed }} \\
\text { (without } \\
\text { Distribution) }\end{array}$ \\
\hline MAPE (\%) & 16.50 & 9.70 & 11.86 & 4.01 & 5.73 & 9.03 \\
\hline RMSE(veh/ln/15min) & 73.94 & 41.76 & 52.11 & 18.11 & 26.60 & 40.34 \\
\hline
\end{tabular}

\subsubsection{VOR Consideration in ML Modeling}

As explained in Chapter 3, the developed methodology functions to calculate the $80^{\text {th }}$ and the $95^{\text {th }}$ percentiles were used to estimate the reliability in this study. The used functions were developed based on the regression analysis in the previous study (Hadi et al., 2014) with one-year traffic detector data and event data in 2012 for the I-95 northbound corridor in Miami, as explained in Section 3.2.1.

As the travel time indices are calculated based on the real-world data, they may not be consistent with the modeled values. Therefore, instead of directly using these calculated values in the traffic assignment, the ratios of the $80^{\text {th }}$ and $95^{\text {th }}$ percentile travel 
time indices to the mean travel time index were calculated and these ratios were multiplied with the simulated mean travel times in the assignment to obtain the simulated $80^{\text {th }}$ and $95^{\text {th }}$ percentile travel time indices. These resulting indices are then added to the generalized cost function utilized in the dynamic traffic assignment based on Equation 37.

In Equation 3-8, $a_{3}$ is assumed to be equal to $a_{4}$ assuming that travelers put the same weights on the $80^{\text {th }}$ and $95^{\text {th }}$ percentile travel time reliability. This study assumes that the VOT/VOR ratio is 1.1 based on the assumptions from the SHRP2 C04 project. Based on Equation 3-8 and the above assumptions $\mathrm{a}_{1}, \mathrm{a}_{3}$, and $\mathrm{a}_{4}$ are estimated to be 22,10 , and 10 , which are the total VOT of $\$ 22$ and VOR of $\$ 20$.

Figure 4-19 and Figure 4-20 show the diverted volumes to ML with and without the consideration of VOR, respectively utilizing different modeling tools. As shown in these figures, the results generated from different ML modeling tools are closer to real-world observations when the VOR is considered in the ML modeling.

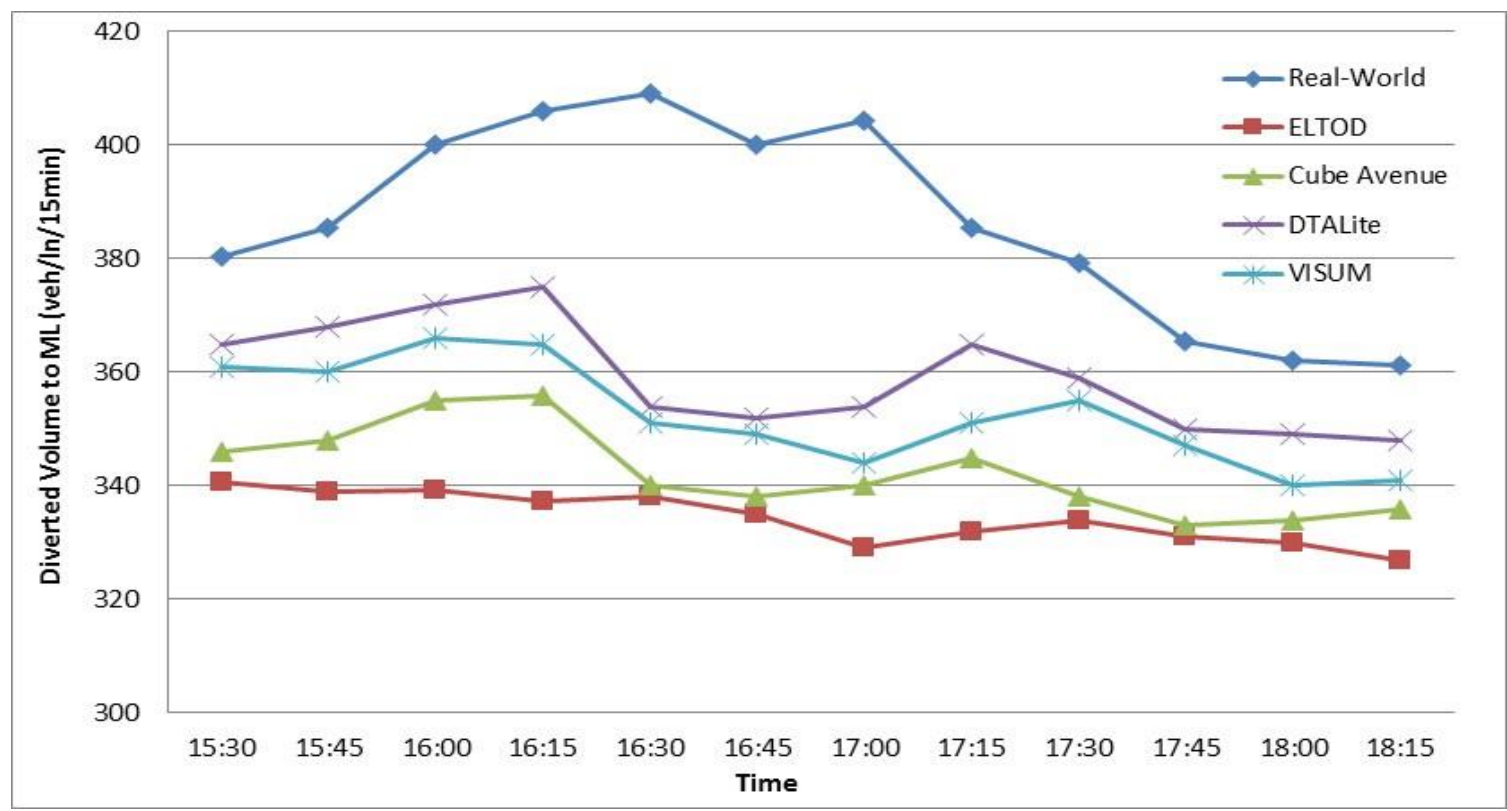

Figure 4-19 Comparison of Diverted Volume to ML without VOR Utilizing Different Tools 


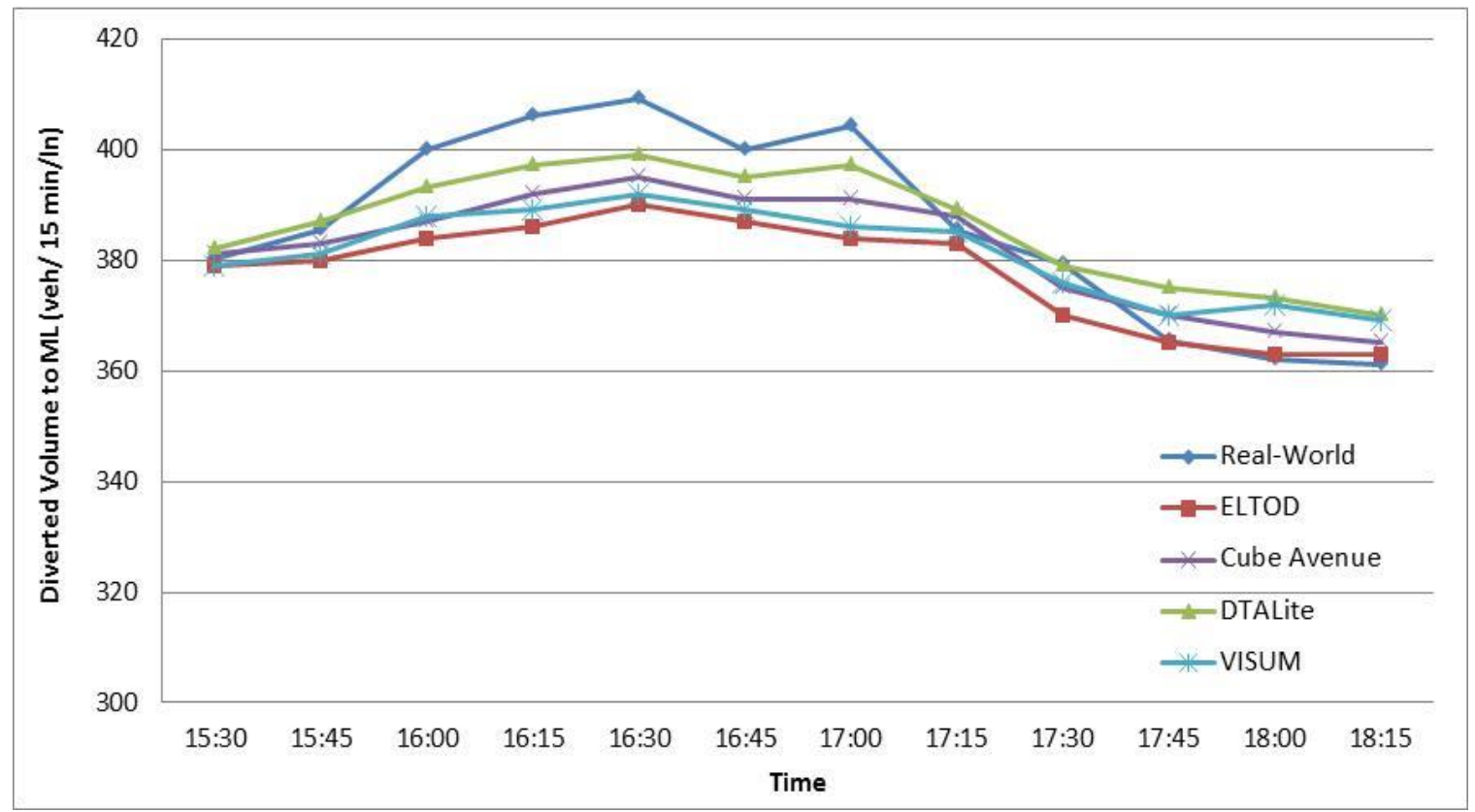

Figure 4-20 Comparison of Diverted Volume to ML with VOR Utilizing Different Tools

The corresponding MAPE and RMSE values to the results presented in Figure 4-19 and Figure 4-20 are summarized in Table 4-8. All results are based on a $\$ 40$ VOT, which was presented in the previous section. The results show that DTALite without the consideration of VOR can produce better results compared to other tools, relative to the real-world diverted volumes to the ML. When the VOR is considered, the results from all tools are significantly improved.

Table 4-8 Goodness-of-Fit Statistics for Diverted Volume Replication with and without the Consideration of VOR

\begin{tabular}{|c|c|c|c|c|c|}
\hline \multicolumn{2}{|c|}{ Goodness-of-Fit Statistics } & ELToD & $\begin{array}{c}\text { Cube } \\
\text { Avenue }\end{array}$ & DTALite & VISUM \\
\hline $\begin{array}{c}\text { With } \\
\text { Consideration } \\
\text { of VOR }\end{array}$ & $\begin{array}{c}\text { RMSE } \\
\text { (veh/ln/15min) }\end{array}$ & 12.00 & 9.18 & 8.23 & 10.77 \\
\cline { 2 - 6 } & MAPE (\%) & 2.29 & 1.96 & 1.89 & 2.27 \\
\hline $\begin{array}{c}\text { Without } \\
\text { Consideration } \\
\text { of VOR }\end{array}$ & $\begin{array}{c}\text { RMSE } \\
\text { (veh/ln/15min) }\end{array}$ & 54.30 & 46.22 & 31.02 & 37.03 \\
\cline { 2 - 6 } & MAPE (\%) & 13.36 & 11.29 & 6.93 & 8.68 \\
\hline
\end{tabular}




\subsubsection{Dynamic Pricing}

The previous analysis assumed a fixed pricing to the modeling of ML. The benefit of modeling the dynamic toll policy used for the I-95 northbound managed lane in Miami, FL instead of a fixed toll value was also investigated in this study. Instead of a fixed toll, the dynamic I-95 ML pricing is defined as a function of maximum traffic density along the managed lanes with the purpose of maintaining a desired level of service, as is done in the real-world. This toll policy was modeled in Cube Avenue using the script language of Cube. The script language in Cube Avenue was used in this research for dynamic pricing in ML modeling. During each time interval in Cube Avenue, the maximum link density is calculated by comparing the densities of all ML links in each direction. Once the maximum density is found, the corresponding toll cost (\$) is obtained by looking up a predefined toll policy table.

Table 4-9 shows the I-95 ML toll policy before March 1, 2014. In order to relieve the congestion along the ML, FDOT D6 increased the minimum toll from $\$ 0.25$ to $\$ 0.50$ and the maximum toll from $\$ 7.00$ to $\$ 10.50$ as shown in Table 4-10. This study aims to test the robustness of the ML modeling to estimate the increase in diversion when changing the managed lane pricing and other policies. Since VISUM and DTALite only allow a fixed toll rate, they are not included in this dynamic analysis.

Table 4-9 I-95 ML Old Toll Policy (Before March, 2014)

\begin{tabular}{|c|c|c|c|c|}
\hline \multirow{2}{*}{ Level Of Service } & \multicolumn{2}{|c|}{ Road Density (veh/mi/ln) } & \multicolumn{2}{c|}{ Toll Cost (\$) } \\
\cline { 2 - 5 } & Minimum & Maximum & Minimum & Maximum \\
\hline A & 0 & 11 & 0.25 & 0.25 \\
\hline B & 12 & 18 & 0.5 & 1.25 \\
\hline C & 19 & 26 & 1.5 & 2.75 \\
\hline D & 27 & 35 & 3 & 3.75 \\
\hline E & 36 & 45 & 3.75 & 6 \\
\hline F & \multicolumn{2}{|r}{} & 6 & 7 \\
\hline
\end{tabular}


Table 4-10 I-95 ML New Toll Policy (After March, 2014)

\begin{tabular}{|c|c|c|c|c|}
\hline \multirow{2}{*}{ Level Of Service } & \multicolumn{2}{|c|}{ Road Density (veh/mi/ln) } & \multicolumn{2}{c|}{ Toll Cost (\$) } \\
\cline { 2 - 5 } & Minimum & Maximum & Minimum & Maximum \\
\hline A & 0 & 11 & 0.5 & 0.5 \\
\hline B & 12 & 18 & 0.5 & 1.55 \\
\hline C & 19 & 26 & 1.5 & 8.5 \\
\hline D & 27 & 35 & 8.5 & 9.5 \\
\hline E & 36 & 45 & 9.5 & 10.5 \\
\hline F & \multicolumn{2}{|c|}{$>45$} & 10.5 & 10.5 \\
\hline
\end{tabular}

Figure 4-21 shows the toll-density curves of the ML with the old and new toll policies for the I-95 ML based on utilizing dynamic pricing model in Cube Avenue and ELToD. It should be noted that the observed data in this figure refer to the density estimated from the real-world detector data. It can be seen from this figure that the new policy resulted in a reduction in the density of the managed lanes due to higher toll cost and this was also reflected in the utilized tool results.

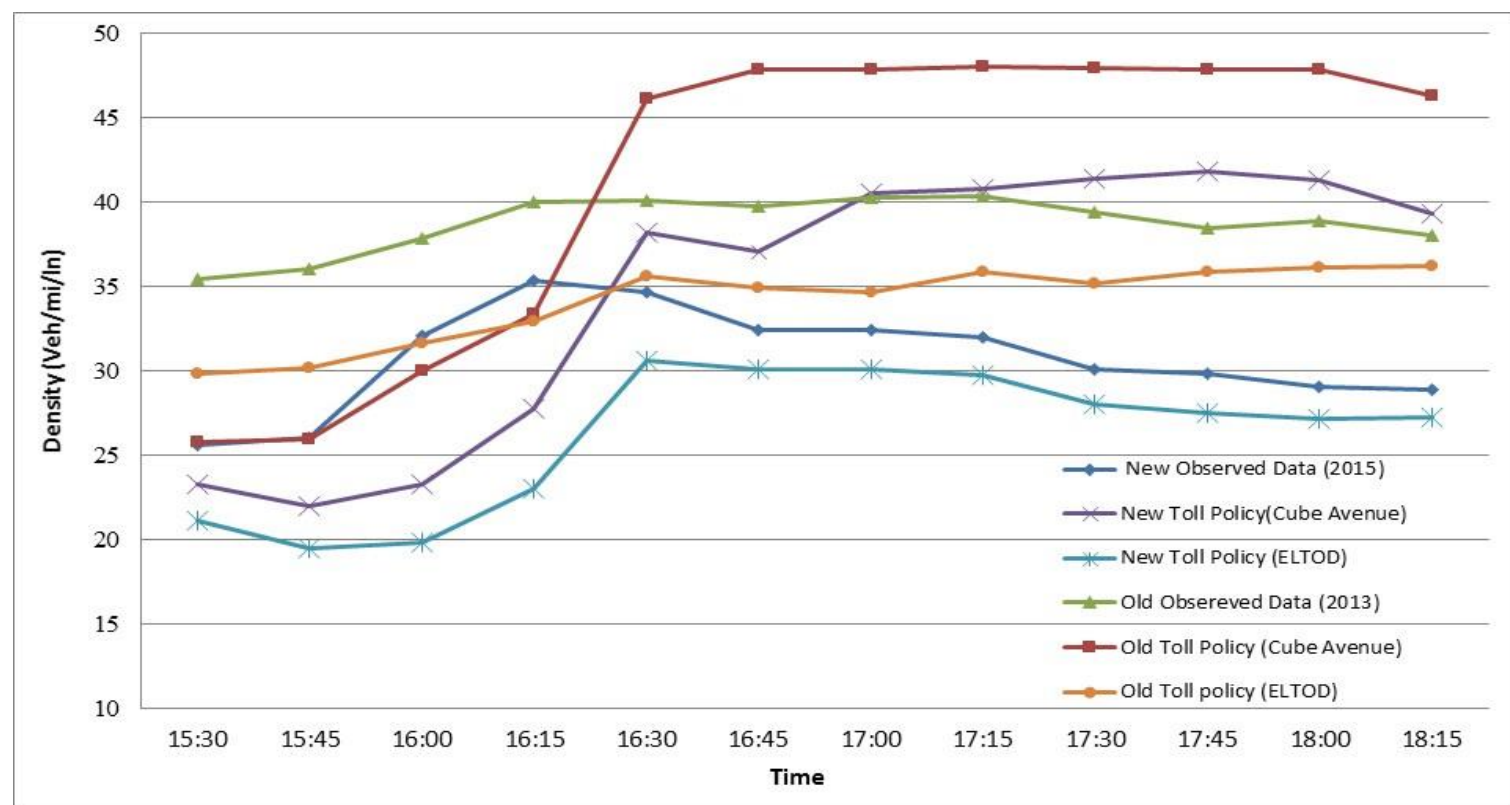

Figure 4-21 Comparison of Toll-Density Curves for Old and New Toll Policies for I-95 Northbound Utilizing Different Tools 
Figure 4-22 presents the corresponding results for the diverted volume to ML when using the old and new toll policies with different tools. It can be seen in this figure that ELToD, a static assignment-based ML model, underestimates the diverted volumes to the ML based on the old toll policy and the new toll policy However, the dynamic assignment-based managed lane model implemented in Cube Avenue can produce the diverted volume results similar to the real-world measurements. It can also be seen that the differences between the diverted volume to the ML in the simulated and observed data before and after implementing the policy are the same, which indicates that the ML models in ELToD and Cube Avenue are robust in capturing the impacts of toll policy changes.

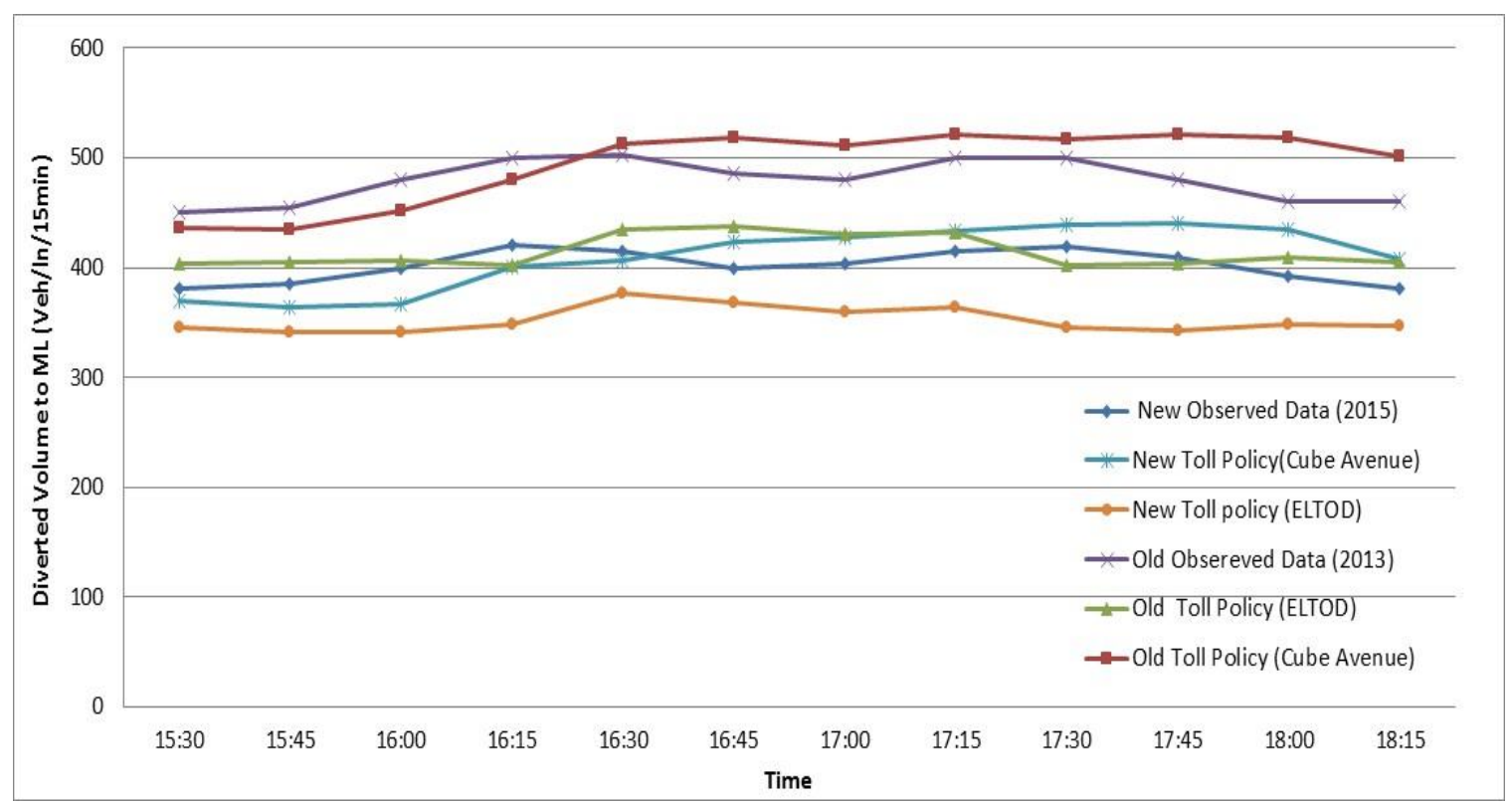

Figure 4-22 Comparison of Diverted Volume to ML Using Old and New Toll Policies for I95 Northbound Utilizing Different Tools

The results of the percentage share of ML volumes compared to total volumes along the corridor utilizing different tools for different toll scheduled policies are presented in Table 4-11. The percentage of ML share in this table is calculated as the 
number of vehicles diverted to ML divided by total number of vehicles traveling along the corridor at the entrance of managed lanes.

Table 4-11 Variation of Percentage of ML Share with Respect to toll Scheduled Policy Utilizing Different Tools (\%)

\begin{tabular}{|c|c|c|c|c|c|c|}
\hline \multirow{2}{*}{ Time } & \multicolumn{3}{|c|}{ Old Toll Policy } & \multicolumn{3}{c|}{ New Toll Policy } \\
\cline { 2 - 7 } & ELToD & Cube Avenue & Real-World & ELToD & Cube Avenue & Real-World \\
\hline $\mathbf{1 5 : 3 0}$ & 32.44 & 35.10 & 36.21 & 27.78 & 29.70 & 30.60 \\
\hline $\mathbf{1 5 : 4 5}$ & 32.57 & 34.97 & 36.61 & 27.52 & 29.28 & 31.00 \\
\hline $\mathbf{1 6 : 0 0}$ & 32.71 & 36.34 & 38.62 & 27.42 & 29.53 & 32.18 \\
\hline $\mathbf{1 6 : 1 5}$ & 32.32 & 38.68 & 40.23 & 28.04 & 32.20 & 33.82 \\
\hline $\mathbf{1 6 : 3 0}$ & 35.02 & 41.31 & 40.47 & 30.30 & 32.69 & 33.42 \\
\hline $\mathbf{1 6 : 4 5}$ & 35.17 & 41.66 & 39.10 & 29.61 & 34.03 & 32.18 \\
\hline $\mathbf{1 7 : 0 0}$ & 34.70 & 41.20 & 38.62 & 28.98 & 34.44 & 32.53 \\
\hline $\mathbf{1 7 : 1 5}$ & 34.77 & 42.00 & 40.23 & 29.30 & 34.92 & 33.42 \\
\hline $\mathbf{1 7 : 3 0}$ & 32.39 & 41.59 & 40.23 & 27.78 & 35.32 & 33.73 \\
\hline $\mathbf{1 7 : 4 5}$ & 32.53 & 41.98 & 38.62 & 27.63 & 35.48 & 32.93 \\
\hline $\mathbf{1 8 : 0 0}$ & 32.93 & 41.68 & 37.01 & 28.01 & 35.00 & 31.56 \\
\hline $\mathbf{1 8 : 1 5}$ & 32.63 & 40.39 & 37.01 & 27.90 & 32.83 & 30.60 \\
\hline
\end{tabular}

\subsection{Modeling of Cooperative Adaptive Cruise Control (CACC) in ML Utilizing} MRM

\subsubsection{Capacity Impact Estimation based on Microscopic Simulation}

As stated earlier in the methodology chapter, the lane capacity as a function of the proportions of CACC vehicles in the traffic stream was estimated by Shladover et al. (2012), using microscopic simulation. The time gap distribution that was used in a realworld field test were used as inputs into a car-following model in the AIMSUN microscopic simulation tool. The parameters of the car-following models are the distance between vehicles, speeds of both the preceding and following vehicles, and vehicle lengths. 
The scenario with all manually driven vehicles was used as the base scenario. The simulation of this base scenario resulted in an average capacity of $2,018 \mathrm{veh} / \mathrm{ln} / \mathrm{hr}$, in accordance with the Highway Capacity Manual (HCM) estimates.

The desired time gaps of ACC-equipped and CACC- equipped vehicles used in the simulation were identified from the gaps selected by drivers in the field test, as listed below:

- $\quad$ ACC: $31.1 \%$ at $2.2 \mathrm{sec}, 18.5 \%$ at $1.6 \mathrm{sec}$, and $50.4 \%$ at $1.1 \mathrm{sec}$

- $\quad$ CACC: $12 \%$ at $1.1 \mathrm{sec}, 17 \%$ at $0.9 \mathrm{sec}, 24 \%$ at $0.7 \mathrm{sec}$, and $57 \%$ at $0.6 \mathrm{sec}$

When basic ACC vehicles with the above time gaps were simulated in the traffic stream, the capacity increased within a narrow range from 2,018 veh/ln/hr to 2,100 $\mathrm{veh} / \mathrm{ln} / \mathrm{hr}$, which is close to the base scenario capacity, regardless of the market penetration. This can be explained by noting that drivers of ACC-equipped vehicles use similar time gap setting to the time gaps that they set when they drive manually (the base scenario). However, when various combinations of manually driven and CACC vehicles were considered, the results showed that the capacity grew slowly the CACC market penetration was low, and then it grew more rapidly as the market penetration increased further. With $100 \%$ of CACC vehicles in the traffic, the lane capacity would increase from $2,018 \mathrm{veh} / \mathrm{ln} / \mathrm{hr}$ to $3,970 \mathrm{veh} / \mathrm{ln} / \mathrm{hr}$, which means $97 \%$ capacity increase, compared to the base capacity. Figure 4-23 shows the percentage of lane capacity increases with the different market penetration of CACC vehicle based on the results from Shladover et al. (2012). 


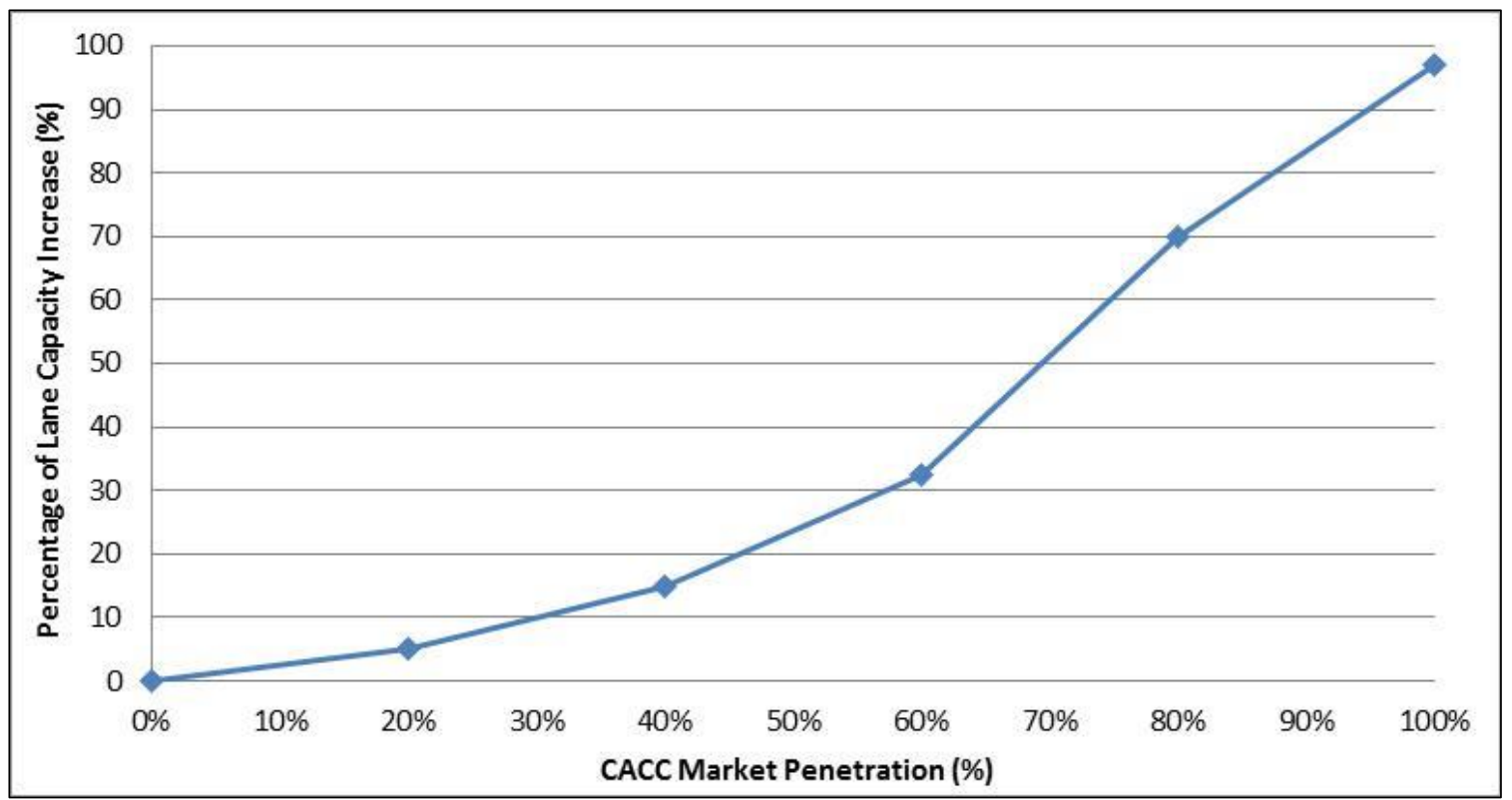

Figure 4-23 Impacts of CACC Proportion on Lane Capacity (Shladover et al., 2012)

The results from microscopic simulation, according to Shladover et al. (2012), indicate that the capacity is not significantly impacted by the introduction of ACCequipped vehicles into the traffic stream. However, the increase in the percentage of CACC technology significantly increases the capacity. If preferential treatment is given to CACC vehicles when using ML, this is expected to result in an increase in their percentage on ML and thus is expected to improve the mobility benefits of these devices by having more CACC vehicles concentrated on the lanes, which is a condition for capacity improvements. These potential impacts are investigated in this research by using demand forecasting modeling based on STA and mesoscopic simulation modeling based on DTA using the results from the microscopic simulation. This analysis approach can be considered as a multi-resolution modeling (MRM) approach since it uses results from macroscopic, mesoscopic, and microscopic models. 


\subsubsection{Modeling the Impacts of CACC Vehicle Based on Macroscopic and Mesoscopic}

\section{Simulation}

This study examined the assessment of the impacts of CACC vehicle technologies on the performance of ML and GPL in the exploration network using macroscopic and mesoscopic models based on capacity estimates from microscopic simulation models by Shladover et al. (2012), as described in the previous section. The assessment was based on the capacity estimated by Shladover et al. (2012). This capacity was coded as a variable in demand forecasting modeling tool with macroscopic traffic model and a mesoscopic simulation-based DTA tool. The capacity was allowed to vary in each assignment iteration, as a function of the percentages of CACC in traffic streams in that iteration, according to the findings from the microscopic simulation study.

Macroscopic traffic flow-based STA and mesoscopic simulation-based DTA were used to assess diversions between GPL and ML, in response to different CACC-equipped vehicle market penetrations and different ML strategies in the exploration network, which includes eight miles of the northbound direction of the I-95 freeway corridor in Miami, FL with 288 nodes, 303 links and 57 zones. As described earlier in the methodology section, three different user groups of demand matrices were used in the model: Tolled Vehicles, Shared Ride of three or more occupants (SRP3), and Truck. SRP3 were allowed to use the ML without any cost or restriction, and trucks were not allowed to use ML.

In this research, the mobility impacts of CACC-equipped vehicles were modeled first based on the macroscopic traffic flow-based STA implemented in the SERPM. The same scenarios were also modeled in Cube Avenue, a mesoscopic simulation-based DTA 
tool. The results from STA and DTA were aggregated into peak period values for comparison purposes.

Although various projections have been reported in the literature to predict the market penetration of CACC vehicles from now until year of 2040, there is no consent yet on one specific projection. Therefore, a sensitivity analysis was conducted in this study to examine the impacts of CACC vehicle market penetration. Four values of market penetration were considered in the study: $0 \%, 20 \%, 60 \%$, and $100 \%$. Also, a tolling policy was tested in this study to give incentive to the vehicles equipped with CACC and encourage them to use the managed lanes by providing toll pricing discount to these vehicles. The rationale behind this policy is that for a given demand, the maximum managed lane throughput is expected to increase as the percentage of CACC vehicles traveling along the managed lanes increases due to the smaller gaps between vehicles, which may help reduce congestion on the ML and along the parallel GPL. In addition, two demand levels were included in the analysis; one corresponding to the existing travel demand, and another with $100 \%$ increase in demand to represent an extreme case of increase in future demand.

The impacts of the CACC market penetration, toll discount rates and demand level on the portion of travelers that select the managed lanes under different scenarios was examined first using STA and the corresponding results are displayed in Figure 4-24. 


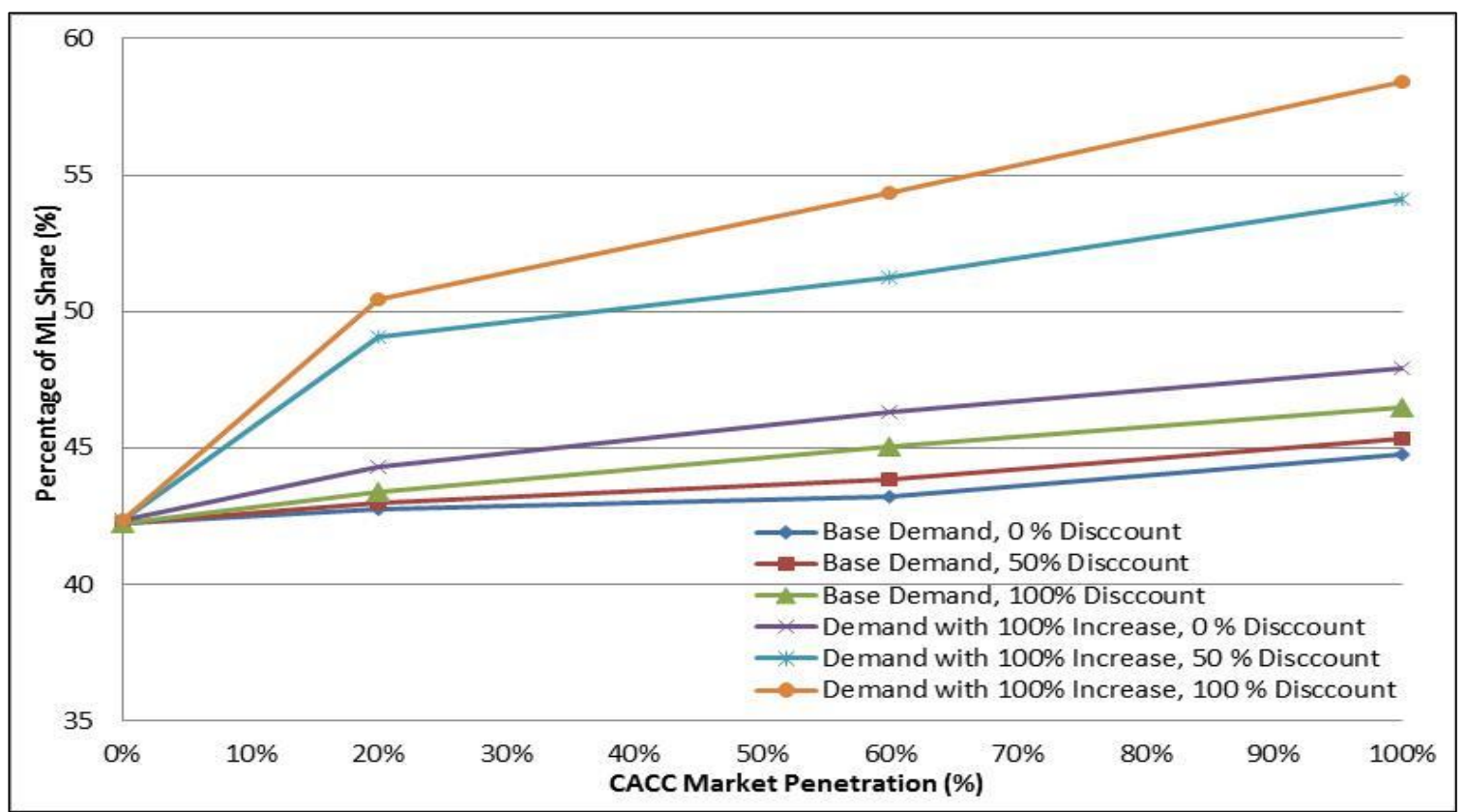

Figure 4-24 Variation of Percentage of ML Share with Respect to CACC Market Penetration Using STA

As shown in Figure 4-24, based on the STA analysis, the percentage of ML share increases with the increase in the CACC market penetration for a given demand level and toll discount. It should be noted that the percentage of ML share in this figure is calculated as the number of vehicles diverted to managed lanes divided by the total number of vehicles traveling along the corridor at the entrance of managed lanes. The managed lane has two entrance points close to each other at the beginning of the system and the ML and GPL are separated until the end of the system. There are many ramps feeding the system and the higher percentages of vehicles that can be diverted to ML at the beginning of the system. It should be noted that due to the separation between ML and GPL, on-ramp vehicles have to use the GPL and cannot use the ML. In addition, all vehicles at the entrance of the system that are destined to off-ramps on the study segments are not able to use the ML due to the fact that they are not able to exit the ML 
to get to the off-ramps. Thus, the separation between GPL and ML creates a maximum limit on the number of vehicles that are able to use the ML.

With the base demand, this increase is from about $42 \%$ for $0 \%$ CACC market penetration to about $45 \%-46 \%$ for $100 \%$ CACC market penetration depending on the values of the toll discount rate. It appears that with the current demand, at small market penetrations of $\mathrm{CACC}$, the increase in capacity due to CACC is small according to the relationship between the capacity and CACC vehicle proportion used in this study. Thus, as incentives are given to CACC vehicles and they divert to the ML in the assignment iterations.

At higher market penetrations and with the base demand level, the capacity increase on the GPL and ML is significant, reducing the congestion on the GPL and the ML, and thus results in the motivation to shift to the ML. As the demand doubled with an increase of $100 \%$ in demand, the percentage of the ML share shows a significant increase from about $42 \%$ for $0 \%$ CACC market penetration to $48 \%-58 \%$ for all CACC toll discount rates when the CACC market penetration is $100 \%$. Also, it can be seen that the increase in the toll discount rate for vehicles equipped with CACC can attract more vehicles to use managed lanes, especially when the market penetration of CACC is high. For example, at $60 \%$ market penetration, the ML share increased from $46 \%$ to about $54 \%$ when $100 \%$ discount is provided.

Figure 4-25 presents the DTA model-based results of the percentage share of ML volumes, compared to the total volumes along the corridor. Again, the percentage of ML share in this figure is calculated as the number of vehicles diverted to ML divided by the total number of vehicles traveling along the corridor at the entrance of managed lanes. It 
is interesting to note that the results in this figure show similar trends to those shown in Figure 4-24, as described earlier. However, the increase in the ML percentage share with the increase in demand and toll discount is more in the DTA modeling, compared to the STA modeling, which reflects the ability of DTA to better model congestion impacts. Compared to the STA modeling, simulation-based DTA considers the capacity constraint and the queue propagation, and thus produces more realistic results.

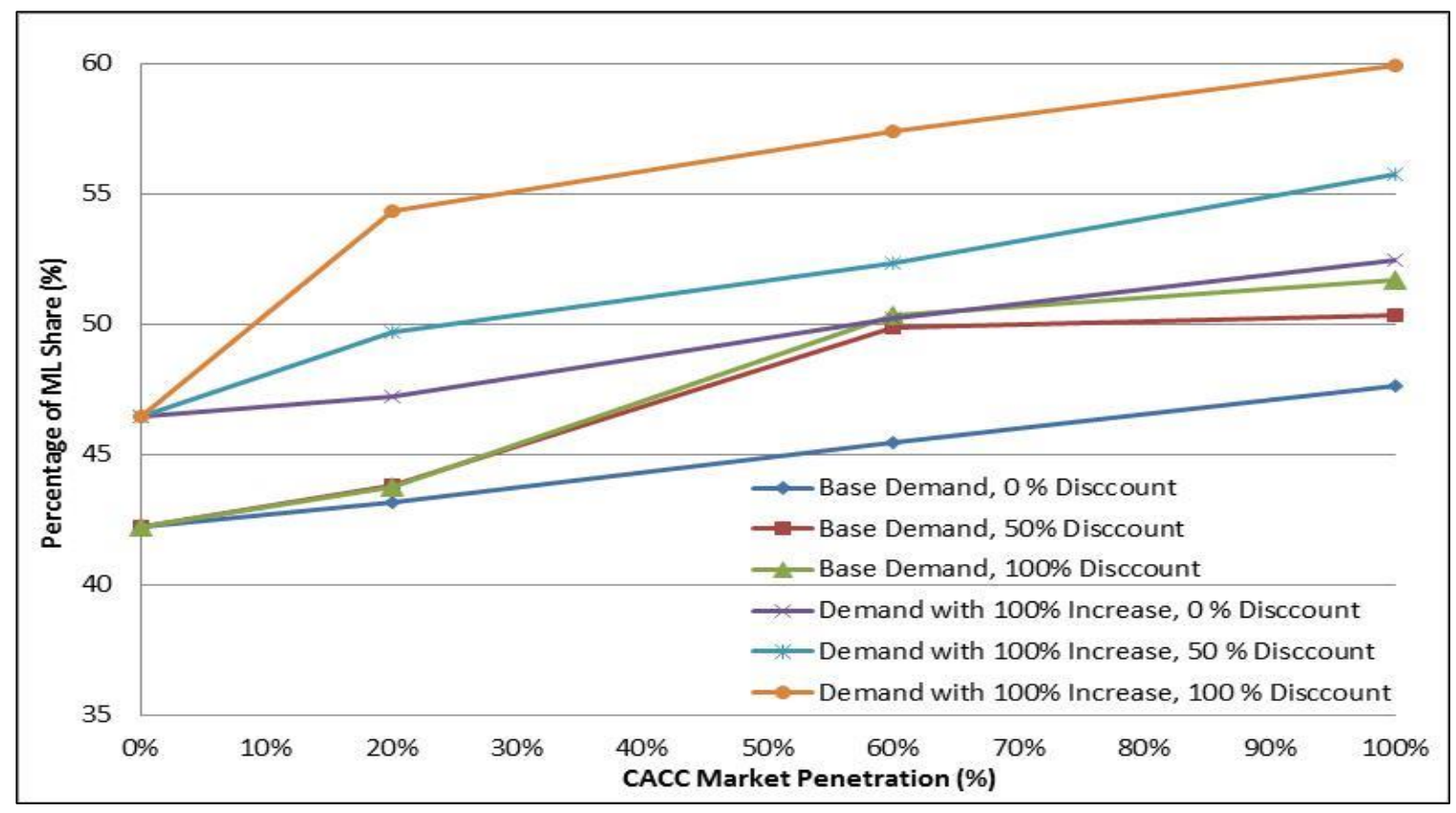

Figure 4-25 Variation of Percentage of ML Share with Respect to CACC Market Penetration Using DTA

It can be seen from Figure 4-25 that for the scenarios with the base demand, the percentage of ML share can be increased from $42 \%$ when the CACC market penetration is $0 \%$ to $47 \%-52 \%$ for $100 \%$ CACC market penetration, which is higher than the percentage share of $45 \%$ - $46 \%$ resulted from the STA modeling. Similarly, for the high demand scenario (100\% increase in demand), the percentage of ML share is about $42 \%$ for 0\% CACC market penetration, and is about $48 \%$ - 58\% for 100\% CACC market penetration based on the STA results. These values are 52\% - 60\% for 100\% CACC 
market penetration according to the DTA modeling results. It should be noted that the high demand causes higher congestion, which gives incentives for more drivers to use the ML, and therefore results in a higher ML percentage share. Another example comparison is that $60 \%$ CACC market penetration with the base demand, proving $100 \%$ discount, increases market penetration from $43 \%$ to $45 \%$ according to the STA, and from $45 \%$ to $50 \%$ according to the DTA.

One of the bottleneck locations was at the NW 103rd St. interchange along the study corridor as identified earlier in Section 4.1.2. Figure 4-26 presents the corresponding worst speed at this location along the GPL during the analysis period, according to the STA analysis.

The results in this figure show that the speed at the bottleneck location increases when the market penetration of CACC is increased and the discount rate is increased because of the higher roadway capacity associated with the higher percentage of CACC and the increasing shift to ML. When the CACC market penetration is less than $20 \%$, the toll discount does not show a significant impact on the bottleneck location speed. This can be explained again by the earlier discussion that with small market penetrations of CACC the increase in capacity due to CACC is small according to the relationship between capacity and CACC vehicle proportion used in this study. Thus, as incentives is given to $\mathrm{CACC}$ vehicles and they divert to $\mathrm{ML}$ in the assignment iterations, the congestion on the ML increases, and causes the CACC vehicle to shift back to the GPL. However, an about $5 \mathrm{mi} / \mathrm{h}$ increase in the speed at bottleneck can be observed from Figure 4-26 due to toll discounts at the higher CACC market penetrations. 
When the demand is increased by $100 \%$, the speed at the bottleneck location becomes lower compared to the scenarios with the base demand. However, the changes of bottleneck location speed with respect to the CACC market penetration and toll discount rates are similar for these two demand levels.

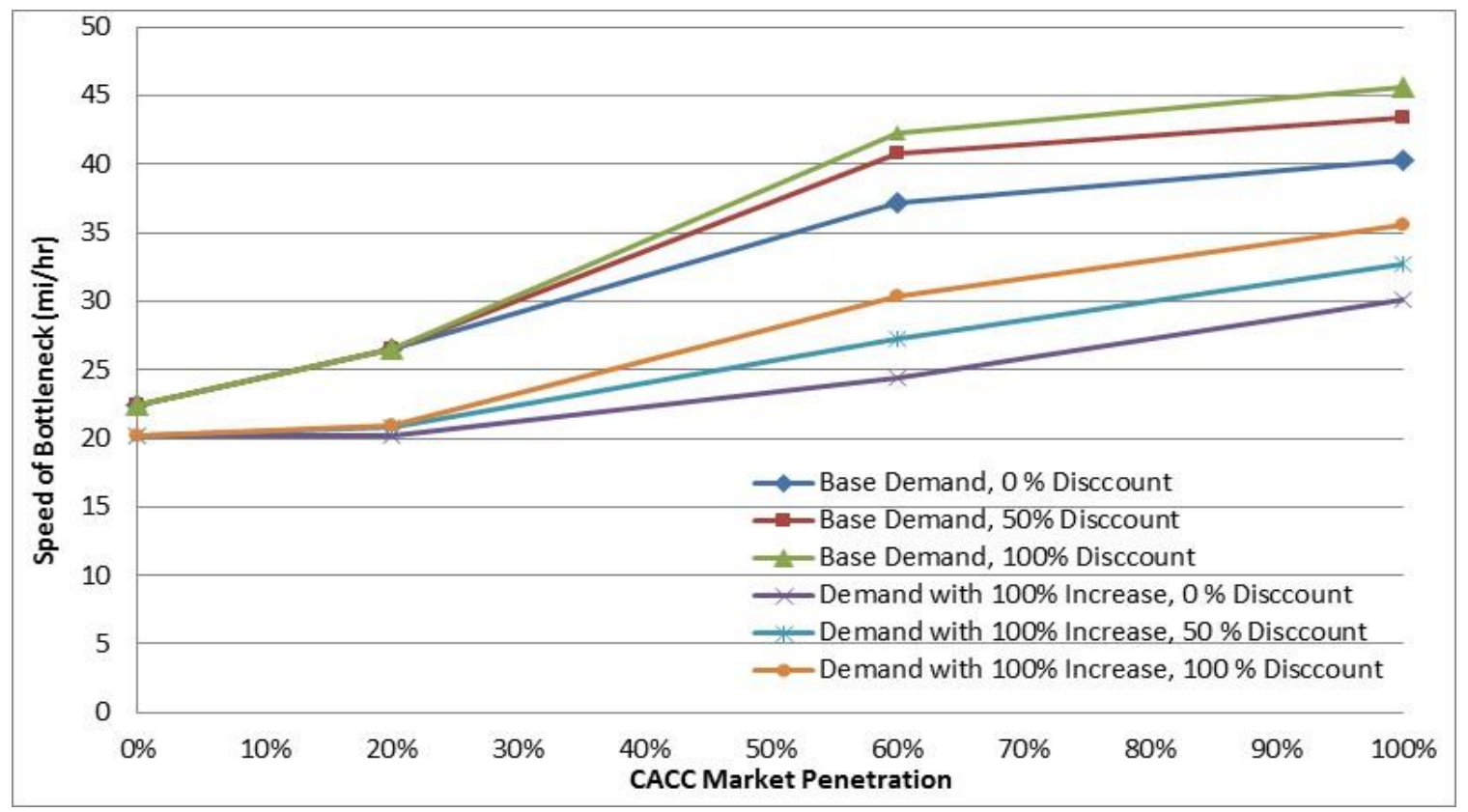

Figure 4-26 Variation of Speed at Bottleneck Location with Respect to CACC Market Penetration Using STA

Figure 4-27 presents the corresponding DTA analysis for the speed at the worst bottleneck location, along the GPL. As shown in this figure, the bottleneck location speed is improved with the increase in the CACC market penetration, especially at the high CACC market penetrations. 


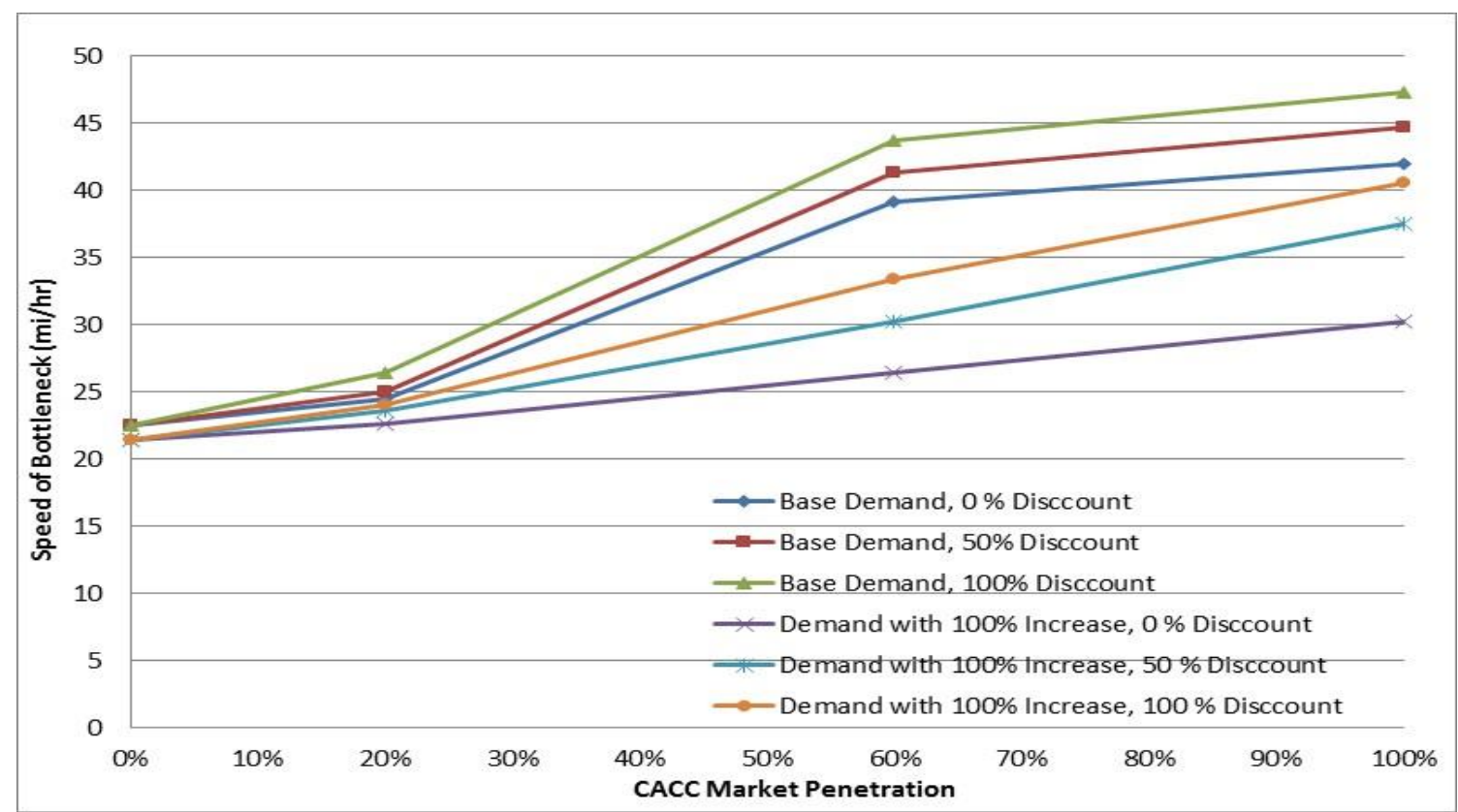

Figure 4-27 Variation of Speed at Bottleneck Location with Respect to CACC Market Penetration Using DTA

A close comparison of the results in Figure 4-26 and Figure 4-27 reveals that for the scenarios with the base demand, the implementation of different toll discounts can cause about $5 \mathrm{mi} / \mathrm{h}$ difference in the bottleneck location speed given a CACC market penetration of $100 \%$ based on both STA and DTA simulations. However, increasing the toll discount rate from $0 \%$ to $100 \%$ at the $100 \%$ CACC market penetration can improve the bottleneck location speed from $30 \mathrm{mi} / \mathrm{h}$ to $40 \mathrm{mi} / \mathrm{hr}$ according to the DTA analysis, as shown in Figure 4-27, while such improvement in bottleneck location speed is only from $30 \mathrm{mi} / \mathrm{hr}$ to $35 \mathrm{mi} / \mathrm{hr}$ according to the STA results in Figure 4-26. This is consistent with the impacts of the toll discount rate on the percentage of ML share utilizing STA and DTA, as shown in Figure 4-24 and Figure 4-25, in which DTA modeling results show more vehicles using the ML than the STA modeling results, which causes a higher speed at the bottleneck location in DTA modeling compared to STA modeling. 
The results of this section demonstrate the benefit of using results from tools with different levels of modeling to support each other's analyses. In general, the trends obtained based on results from the STA modeling of advanced vehicle technologies in terms of the market share of traffic in ML and the reduction in congestion on GPL are consistent with those obtained from DTA. However, DTA results show more significant shifts due to better modeling of traffic congestion. The results also show that providing toll incentives for CACC-equipped vehicles to use express lanes is not beneficial at a lower market penetration due to the small increase in capacity with these market penetrations. Such incentives are beneficial at higher market penetrations, particularly with higher demand levels.

\subsection{Summary}

The MRM framework developed in Chapter 3 was applied in this study to model managed lanes and the impacts of associated operational and pricing strategies. To achieve this purpose, the methodology described in Chapter 3 was applied to a network, which has already been modeled and calibrated with a mesoscopic simulation-based DTA tool (Cube Avenue) by Hadi et al. (2013), to explore and examine the concepts and the components of the MRM in this study.

A number of modeling tools used in this case study includes: Express Lanes Time of Day (ELToD) coded in Cube and VISUM as the macroscopic tools, and Cube Avenue and DTALite as the mesoscopic DTA tools. The first step was to convert the network and demand data from the calibrated network in Cube by Hadi et al. (2013) to VISUM and DTALite utilizing a set of shape files and demand matrices. 
This research examined the impacts of the calibrated and uncalibrated TFM parameters in the simulation results using different modeling tools. The results showed that the use of calibrated TFM parameters reduces the deviations from the real-world speeds. Both DTALite and VISUM tools produce better results than Cube Avenue. Using the Ackcelik function with Cube Avenue improved the results.

The power of ODME modules in Cube, VISUM, and DTALite tools were tested and compared to optimize the demand matrices estimation in comparison to the real world measures in this research. The results showed that DTALite can produce better volume results than the other two tools. A better demand matrix used as input to the ODME process (such as the previously calibrated demand matrices) can produce a more realistic replication of real-world volume counts, compared to utilizing simple factorized demand matrices. The implementation of the dynamic ODME in VISUM and DTALite can better capture the queue forming and dissipation than the static assignment-based ODME implemented in Cube, which can result in more accurate volume counts.

The developed MRM framework was applied in this research to model managed lanes and the impacts of associated operational and pricing strategies. The findings from SHRP 2 C04 were applied to set the generalized cost function in DTALite because it allows the users to define the distribution of the value of time among other explored tools in this study. The results showed that the value of time of $\$ 40$ produced the closest results to the real-world diverted volume to ML. These results also confirmed that utilizing a distribution of VOT, instead of a fixed value, produces better correspondence to the realworld diversion. 
In addition, a methodology was proposed to incorporate the impacts of travel time reliability in the selection of managed lanes. The value of 1.1 was assumed for the ratio VOT/VOR in this study. In this research, the $80^{\text {th }}$ and $95^{\text {th }}$ percentile travel time reliability were estimated for the modeling of ML. The results confirmed that there are significant differences between simulated volumes to ML when VOR is not added to the generalized cost function.

In terms of the dynamic pricing approach in the ML modeling, the dynamic I-95 ML pricing is defined as a function of maximum traffic density along the managed lanes with a purpose of maintaining a desired level of service, as is done in the real-world. The results showed that the dynamic assignment-based managed lane model implemented in Cube Avenue can produce closer results of diverted volume to the real-world measurements. However, ELToD as a static assignment-based ML model underestimates the diverted volumes to the ML based on the old toll policy and the new toll policy.

Eventually, the developed MRM framework was applied in the assessment of the impacts of CACC vehicle technologies on the performance of ML and GPL utilizing macroscopic and mesoscopic models based on capacity estimates from microscopic simulation models. The capacity was allowed to vary in each assignment iteration, as a function of the percentages of CACC in traffic streams in that iteration. The trends obtained based on results from the macroscopic simulation are consistent with those obtained from mesoscopic simulation-based DTA. However, DTA results show more significant shifts due to better modeling of traffic congestion. The results also show that providing toll incentives for CACC-equipped vehicles to use express lanes is not 
beneficial at a lower market penetration due to the small increase in capacity with these market penetrations.

\section{CHAPTER 5}

\section{CONCLUSIONS AND RECOMMENDATIONS}

This study proposes methods for selecting and combining tools with different analysis resolutions for modeling Managed Lanes (ML) and the associated advanced strategies. The methods show how data analysis support tools and transportation system modeling tools can be integrated for an effective modeling of ML. This research also demonstrates how a Multi-Resolution Modeling (MRM) approach can be applied to assess the operations of Adaptive Cruise Control (ACC) and Cooperative Adaptive Cruise Control (CACC), as representatives of Autonomous Vehicles (AVs) and Connected Vehicles (CVs) on ML with different incentives (preferential treatments), pricing strategies, and access restrictions. The following subsections are the conclusions based on the results of this research and the recommendations for future works.

\subsection{Conclusions}

A number of tools with different levels of modeling have been developed to assess ML strategies. Selecting and combining these tools for modeling of congested conditions and advanced strategies such as managed lanes are the challenging tasks for transportation modelers. A critical component of the MRM framework is to select the right tools to meet the modeling requirements. A list of criteria was developed in this research for the assessment of simulation tools to model ML with consideration of 
$\mathrm{AVs} / \mathrm{CV}$ s. These criteria can be used by agencies to allow general comparisons of various modeling tools to meet the needs of the specific modeling problem.

Among the analysis support tools, this study identified automatic data conversion between tools with different resolutions, as a critical component needed for the success of MRM frameworks. Such tools should automatically convert the input/output data from one tool format to another. To demonstrate the benefits of this type of tools, this research utilized NEXTA, an open-source tool that can convert the network shape files and demands from regional demand models to inputs accepted by other modeling tools. This tool was successfully used in this study to support the research activities by converting the inputs and/or outputs to formats acceptable by different tools.

The calibration of simulation and Dynamic Traffic Assignment (DTA) tools is another important component of MRM that requires analysis support tools and methods. With DTA-based models, the calibration process requires iterations between the adjustments of supply, demands, and assignment input parameters. Supply calibration of macroscopic and mesoscopic models involves estimating the segment's capacity, freeflow speeds, jam density, and other Traffic Flow Model (TFM) parameters. The quality of the calibration was assessed in this research, based on the degree of deviation between simulated and real-world measurements, as measured by the Mean Absolute Error (MAE), Mean Absolute Percentage Error (MAPE), Root-Mean-Square Error (RMSE), and

R-Squared $\left(R^{2}\right)$.

The supply calibration performed in this study illustrates the importance of specifying the capacity and jam density parameters based on detector measurements in 
macroscopic and mesoscopic simulation-based DTA tools. The effects of calibrating these parameters in a number of tools were assessed and compared. The results showed that the use of the calibrated capacity and jam density in the TFM model improves the simulation results, and reduces the deviations from the real-world speeds.

The trip matrix estimation is an important step in the assignment process. DTA requires time-dependent trip matrices in short time intervals (e.g., 15 minutes or 30 minutes). Therefore, an Origin-Destination Matrix Estimation (ODME) process is needed to estimate the trip tables for short intervals based on an initial matrix obtained from the regional demand model and field data. The performance of ODME modules in Cube, VISUM, and DTALite tools were tested and compared to optimize the demand matrices estimation in comparison to the real world measures in this research. The results showed that DTALite ODME process can produce better link volume results than the other two tools although the VISUM software results are close. It was also found that the quality of the O-D estimation is dependent on the quality of the initial O-D matrix that significantly affects the ODME process in all tools. The advantages of the dynamic ODME in VISUM and DTALite compared to the static ODME in Cube were clearly illustrated in this dissertation.

The results of this research also demonstrated the importance of specifying the parameters of the generalized cost function including the correct VOT and the incorporation of VOR that have not been commonly used in traffic assignment practices. These two parameters are the statistical distribution of the VOT, instead of the fixed value of VOT, and the utilization of VOR in the generalized cost function of the assignment. The results showed that the VOT of $\$ 40$ produced the best results in terms of 
predicting the utilization of ML. This value is much higher than the value of time commonly used in the assignment practices. For example, the VOT of $\$ 13.3$ used in the SERPM model. The results from this research also confirmed that utilizing a distribution of VOT, instead of a fixed value, produces better predictions of real-world utilization of ML.

The VOR essentially represents travelers' willingness to pay for the reduction in travel time variability. Previous researches on the estimating reliability either used a scenario-based approach that was too time-consuming to incorporate in the assignment iterations, or used a simplified approach that did not account for important factors that affect unreliability such as incidents, work zones, and bad weather. In this study, an innovative methodology was proposed to assess the travel time reliability in the traffic assignment process associated with managed lane modeling. The method was based on regression equations developed as part of the SHRP 2 L03 project and later calibrated for the case study corridor (I-95 corridor in Miami) in the SHRP 2 L38C project. According to these equations, the travel time reliability is estimated as functions of demand/capacity (d/c) ratio, lane hour lost due to incidents and work zones, and weather conditions. The equation allows the prediction of various percentile travel time index. In this research, the VOT/VOR ratio was assumed to be of 1.1 according to SHRP2 C04's findings. It was also assumed that travelers put the same weight on the $80^{\text {th }}$ percentile (worst four days in 20 days) and the $95^{\text {th }}$ percentile travel time (worst one day in 20 days) reliability for the modeling of ML. However, this can be modified in the future if new results become available. The results showed that there are significant differences between the simulated and real-world volumes of ML when the VOR is not added to the generalized cost 
function. These results highlighted the importance of utilizing the VOR in the generalized cost function.

This study also investigated weather ML modeling is able to predict shifts in ML volumes when the tool schedule is changed by transportation agencies to divert traffic out of the managed lanes to improve their performance. The results showed that when emulating the dynamic congestion pricing in the model, DTA-based tools produce volume diversions that are comparable with real-world data. However, static assignment models were less successful to predict the exact volumes but they also produced reasonable results in terms of the amount of shift to ML.

Finally, the developed MRM approach, with the selected parameters as described above, was implemented to evaluate the impacts of advanced vehicle technology. For this purpose, this research assessed the impacts of ACC and CACC vehicle technologies on ML and GPL using static and dynamic assignment models based on capacity estimates from microscopic simulation models. The lane capacity as a function of the proportions of CACC vehicles was estimated by Shladover et al. (2012) using microscopic simulation to estimate the effect of different market penetrations of ACC and CACC on freeway capacity. This capacity was then added and coded in static and dynamic traffic assignment models in the present research as a variable that is a function of the percentages of ACC and CACC in the traffic streams in each assignment iteration.

The impacts of CACC were tested in this study combined with a tolling policy that gives incentives to the vehicles equipped with CACC to encourage them to use the managed lanes by providing toll pricing discounts to these vehicles. The rationale behind this policy was that for a given demand, the maximum managed lane throughput was 
expected to increase as the percentage of CACC vehicles traveling along a lane increases due to smaller gaps between vehicles.

The results of this study demonstrated the benefit of using findings based on modeling tools with different levels of modeling to support each other's analyses. In

general, the trends obtained based on results from the static assignment modeling of advanced vehicle technologies in terms of the market share of traffic in ML and the reduction in congestion on GPL are consistent with those obtained from DTA. However, the DTA results showed more significant shifts due to better modeling of traffic congestion. The results also showed that providing toll incentives for CACC-equipped vehicles to use express lanes was not beneficial at lower market penetrations due to the small increase in capacity corresponding to these market penetrations. Such incentives are beneficial at higher market penetrations, particularly with higher demand levels.

\subsection{Recommendations for Future Works}

As mentioned earlier, this study aims to explore the effectiveness of different levels of modeling when used separately and in combination in the modeling of ML. A number of research topics can be recommended to extend the results of this study, as listed below:

- Incorporating microscopic simulation modeling in the developed MRM for the modeling of ML. Microscopic simulation model can be evaluated in the modeling of ML utilizing diverted volume with different simulation tools and using the data from microscopic simulation as inputs to macroscopic and mesoscopic simulation tools. 
- Testing the MRM framework for a larger network that includes parallel arterials to the ML facility to assess the effect of the full MRM framework in the diversion of ML.

- Implementing the explored concepts of the MRM framework to other advanced traffic and demand management strategies such as incident management, smart work zones, and integrated corridor management

- Developing tools to convert real-world data to format that can be used to support the modeling process. This tool should be able to convert the realworld data to the acceptable data format in the different modeling tools.

- Developing tools to support alternative analysis that post processes the outputs from different models and produces additional statistical parameters and visualization for the use in alternative comparisons.

- Assessing the benefits of the VOT and VOR by different user types in the modeling of ML. The stratification of VOT and VOR can be based on user income, trip purpose, vehicle occupancy, or a combination of all. Considering these parameters can replicated the diverted volume to the ML as is done in the real-world.

- Evaluating the impacts of different fixed and dynamic pricing schemes on the utilization of ML using the MRM approach. The benefit of the dynamic pricing schemes can be investigated in different levels of ML modeling.

- Utilizing additional field data to estimate the capacity in different market penetration of $\mathrm{AVs} / \mathrm{CVs}$ to confirm the values used in this study based on the microscopic simulation. 
- Showing how CV/AV modeling can be further enhanced by using microscopic simulation to model weaving, merging, and diverging at the entry and exit points of ML.

\section{REFERENCES}

Abdelghany, A., Abdelghany, K., Mahmassani, H., and Murray, P. (2000). Dynamic traffic assignment in design and evaluation of high-occupancy toll lanes. Transportation Research Record: Journal of the Transportation Research Board, (1733), 39-48.

Akcelik, R. (2003). Speed-Flow and Bunching Relationship for Uninterrupted Flows. Presented at 25th Conference of Australian Institute of Transport Research, University of South Australia.

Arnaout, G. M., and Bowling, S. (2014). A Progressive Deployment Strategy for Cooperative Adaptive Cruise Control to Improve Traffic Dynamics. International Journal of Automation and Computing, Vol. 11, No. 1, pp. 10-18.

Arem, B., and van der Vlist, M. J. (1992). An on-line procedure for estimating current capacity. Technical Report INRO-VVG 1991-17, TNO Institute of Spatial Organization, Delft.

Bassan, S., and Polus, A. (2010). Meaning of Actual Capacity of Freeways and its Estimation. Canadian Journal of Civil Engineering, Vol. 37, No. 1, 2010, pp. 77-87.

Ben-Akiva, M., Palma, A.D., and Kanaroglou, P. (2009). Dynamic Model of Peak Period Traffic with Elastic Arrival Rates. Transportation Science, Vol.20 (3), pp.164-181.

Bifulco, G. N., Pariota, L., and Di Pace, R. (2013). Development and Testing of a Fully Adaptive Cruise Control System. Transportation Research, Part C: Emerging Technologies, Vol.29, pp. 156-170.

Caliper Corporation. (2011). TransModeler 5.0.

Calvert, S. C., Van Broek, T. H., and Van Noort, M. (2012). Cooperative Driving in Mixed Traffic Networks-Optimizing for Performance. IEEE Intelligent Vehicles Symposium, pp. 861-866. 
Cambridge Systematics, Inc. (2012). Value of Travel Time Reliability. Synthesis Report and Workshop Working Paper. Prepared for SHRP 2 Workshops on ValueofTravelTimeReliability.http://onlinepubs.trb.org/onlinepubs/shrp2/L35RF/DraftSy nthesisValuing Reliability.pdf,_Accessed Oct 12, 2013.

Chao, C., Varaiya, P., and Kwon, J. (2005). An Empirical Assessment of Traffic Operations. In Proceedings of International Symposium on Transportation and Traffic Theory.

Chiu, Y.C. (2012) DynusT User's Manual Version 3.0.1. http://dynust.net/wikibin/doku.php?id=start. Accessed May, 2012.

Chiu, Y. C., Bottom, J., Mahut, M., Paz, A., Balakrishna, R., Waller, T., and Hicks, J. (2011). Dynamic traffic assignment: A primer. Transportation Research E-Circular, (EC153).

Chiu, Y. C., Zhou, L., and Song, H. B. (2010). Development and Calibration of the Anisotropic Mesoscopic Simulation Model for Uninterrupted Flow Facilities. Transportation Research Part B 44, pp.152-174.

Chiu, Y. C., and Bustillos, B. (2009). A Gap Function Vehicle Based Solution Procedure for Consistent and Robust Simulation Based Dynamic Traffic Assignment. $88^{\text {th }}$ Annual Meeting of the Transportation Research Board, National Research Council, Washington D.C., USA.

Citilabs (2013). Cube Reference Guide Version 6.4.

Concas, S., and Kolpakov, A. (2009). Synthesis of Research on Value of Time and Value of Reliability. Prepared for Florida Department of Transportation, Final Report Contract No. BD549 46.

Davis, P.K., and Hillestad, R. J. (1998). Experiments in Multi-resolution Modeling. RAND Report. MR1004. RAND Corp.

Davis, L. C. (2004). Effect of Adaptive Cruise Control Systems on Traffic Flow. Physical Review E, Vol. 69, No. 066110, pp. 1-8.

Dynameq, http://www.inro.ca/en/products/dynameq/index.php, Accessed by May 2012.

Decorla Souza, P. (2003). Evaluation of Toll Options Using Quick-Response Analysis Tools: A Case Study of the Capital Beltway. $82^{\text {th }}$ Annual Meeting of the Transportation Research Board, National Research Council, Washington D.C., USA.

DeCorla-Souza, P., and Whitehead, R. E. (2003). The value of pricing the use of roads. Journal of Public Works Management \& Policy, 7(4), pp.267-276. 
Dehghani, Y., Adler, T., Doherty, M. W., and Fox, R. (2003). Development of a New Toll Mode-Choice Modeling System for Florida's Turnpike Enterprise. Transportation Research Record: Journal of the Transportation Research Board, No. 1858, pp. 9-17.

Dervisoglu, G., Gomes, G., Kwon, J., and Varaiya, P. (2009), Automatic Calibration of the Fundamental Diagram and Empirical Observations on Capacity. $88^{\text {th }}$ Annual Meeting of the Transportation Research Board, National Research Council, Washington D.C., USA.

DTALite. (2012). User Manual, http://sites.google.com/site/dtalite/, Accessed May 2012.

Duthie, J., Juri, N. R., Melson, C. L., Pool, C. M., \& Boyles, S. (2012). Guidebook on DTA Data Needs and Interface Options for Integration into the Planning Process. Center for Transportation Research, University at Texas Austin No. 0-6657-P1.

Elefteriadou, L., Martin, B., Simmerman, T., and Hale,D.(2012). Using Microscopic Simulation to Evaluate the Effects of Advanced Vehicle Technologies on Congestion. Department of Civil and Coastal Engineering, University of Florida, Center for Multimodal Solutions for Congestion Mitigation, pp. 87-110.

Fakharian Qom, S., Xiao, Y., Hadi, M., and Al-Deek, H. (2015). Estimation of Freeway Density Based on the Combination of Point Traffic Detector Data and Automatic Vehicle Identification Data. Transportation Research Record: Journal of the Transportation Research Board, No 2484, pp. 110-118.

Fakharian Qom, S., Xiao, Y., and Hadi, M. (2016). Evaluation of Cooperative Adaptive Cruise Control (CACC) Vehicles on Managed Lanes Utilizing Macroscopic and Mesoscopic Simulation. 95 ${ }^{\text {th }}$ Annual Meeting of the Transportation Research Board, National Research Council, Washington D.C., USA, No 16-6384.

Fakharian Qom, S., Xiao, Y., Hadi, M., and Al-Deek, H. (2015). Estimation of Freeway Density Based on the Combination of Point Traffic Detector Data and Automatic Vehicle Identification Data. Transportation Research Record: Journal of the Transportation Research Board, No 2484, pp.110-119.

Federal Highway Administration (FHWA), U.S. Department of Transportation. (2015). Connected Vehicles. Publication No. FHWA-JPO-16-331.

Federal Highway Administration (FHWA), U.S. Department of Transportation. (2013a). The Effective Integration of Analysis, Modeling, and Simulation Tools. Publication No. FHWA-HRT-13-036.

Federal Highway Administration (FHWA), U.S. Department of Transportation. (2013b). Cooperative Adaptive Cruise Control, Human Factors Analysis. Report No. FHWA-HRT13-045. 
Federal Highway Administration (FHWA), U.S. Department of Transportation. (2012a). Traffic Analysis Toolbox Volume XIV: Guidebook on the Utilization of Dynamic Traffic Assignment in Modeling. Report No. FHWA-HOP-13-015.

Federal Highway Administration (FHWA), U.S. Department of Transportation. (2012b). Managed Lanes. http://fhwa.dot.gov/managed lanes.htp. Accessed Oct. 20, 2012.

Federal Highway Administration (FHWA), U.S. Department of Transportation. (2008b). Value Pricing Pilot Program TRUCE 3.0 Users Guide, HOV Lane Performance. http://fhwa.dor.gov/tolling pricing/value pricing/tools/truce model guide.htm, Accessed Oct. 10, 2013.

Federal Highway Administration (FHWA), U.S. Department of Transportation. (2008c). Managed Lanes: A Primer. http://fhwa.dot.gov/managedlanes/managed lanes primer.pdf. Accessed Oct. 20, 2013.

Florian, M., Mahut, M., and Tremblay, N. (2008). Application of a Simulation-Based Dynamic Traffic Assignment Model. European Journal of Operations Research. 189(3), 1381-1392.

Florida Department of Transportation (FDOT), (2013). Southeast Florida Regional Planning Model, v. 6.5.3 (SERPM). Florida Department of Transportation, Florida, http://www.fsutmsonline.net/index.php?/model pages/, Accessed Oct.20, 2013.

Florida's Turnpike Enterprise. (2012). Annual Customer Service Satisfaction Survey.http://www.floridastrnpike.com/downloads/CustomerSurveys/2005custsat_web Accessed Nov 4th, 2013.

Florida's Turnpike Enterprise, (2005) 2005 Annual Customer Service Satisfaction Survey,http://www.floridasturnpike.com/downloads/CustomerSurveys/2005custsat_web.p df, Accessed Nov $4^{\text {th }}, 2013$.

Hadi, M., Xiao, Y., Wang, T., Hu, P., Jia, J., Edelstein, R., and Lopez, A. (2014). Pilot Testing of SHRP 2 Reliability Data and Analytical Products: Florida Pilot Site. SHRP 2 Report.

Hadi, M., Shabanian, S., Ozen, H., Xiao, Y., Doherty, M., Segovia, C., and Ham, H. (2013). Application of Dynamic Traffic Assignment to Advance Managed Lane Modeling. Prepared for Florida Department of Transportation, by the Florida International University Lehman Center for Transportation Research, Miami, Fl.

Hadi, M., Ozen, H., Shabanian, Sh., and Xiao, Y. (2012a). Use of Dynamic Traffic Assignment in FSTUMS in Support of Transportation Planning in Florida. Prepared for Florida Department of Transportation, by the Florida International University Lehman Center for Transportation Research, Miami, Fl. 
Hadi, M., Ozen, H., and Xiao, Y. (2012b). Processes to Guide the Application of Dynamic Traffic Assignment by Transportation Agencies. $91^{\text {th }}$ Annual Meeting of the Transportation Research Board, National Research Council, Washington D.C., USA.

Hadi, M., Xiao, Y., Zhan, C., and Alvarez, P. (2012c). Integrated Environment for Performance Measurements and Assessment of Intelligent Transportation Systems Operations. Prepared for Florida Department of Transportation, by the Florida International University Lehman Center for Transportation Research, Miami, Fl.

Hadi, M., Xiao, Y., Ozen, H., and Alvarez, P. (2008). Evaluation Tools to Support ITS Planning Process: Development of a Sketch Planning Tool in FSUTMS/Cube Environment. Prepared for Florida Department of Transportation, by the Florida International University Lehman Center for Transportation Research, Miami, Fl.

He, R.R., Ran, B., and Choi, K. (2000). Decision Support System for Value Pricing Strategies on I-394 Corridor Network. $79^{\text {th }}$ Annual Meeting of the Transportation Research Board, National Research Council, Washington D.C., USA.

Holyoak, N., and Branko, S. (2009). Benefits of Linking Macro-Demand Forecasting Models and Microsimulations Models. ITE Journal, Vol 79, Issue 10, pp. 30-39

Janson, M., and Levinson, D. (2013). HOT or Not, Driver Elasticity to Price on the MnPASS HOT Lanes. http://nexus.umn.edu/Papers/HOTorNOT.pdf, Accessed Oct. 20, 2013.

Jia, A., Williams, B. M, and Rouphai, N. M. (2010). Identification and Calibration of Site Specific Stochastic Freeway Breakdown and Queue Discharge. Transportation Research Record, Journal of the Transportation Research Board, No. 2188, 2010, pp. 148-155.

Kerns, R., and Paterson, D. (2011). Using a Simulation Environment for Dynamic Toll Analysis., http://www.caliper.com/Library/simulation-methods-foranalyzing-managedlanes.htm. Accessed 2011.

Kesting, A., Treiber, M., Schönhof, M., and Helbing, D. (2007). Extending Adaptive Cruise Control to Adaptive Driving Strategies. Transportation Research Record: Journal of the Transportation Research Board, No. 1, pp. 16-24.

Kockelman, K., Fagnant, D., Nichols, B., and Boyles, S. (2012). A Sketch-Planning Toolkit for Evaluating Highway Transportation Projects. Prepared for Texas Department of Transportation, by the University of Texas at Austin, Austin, TX.

Lee, S. (2014). "Applications of TRANSIMS in Transportation. Procedia Computer Science, Vol.32, pp. 769-773.

Li, J., and Govind, S. (2003). An Optimization Model for Assessing Pricing Strategies of Managed Lanes. $82^{\text {th }}$ Annual Meeting of the Transportation Research Board, National Research Council, Washington D.C., USA. 
Lyman Ott, R., and Longnecker, M. (2001). An Introduction to Statistical Methods and Data Analysis. Texas A\&M University: pp. 445-450.

Mahmassani, H.S., Dong, J., Kim, J. (2009). DYNASMART-P 1.5 User's Guide and Programmer's Guide. Transportation Center, Northwestern University.

Mahut, M., Florian, M., and Tremblay, N., (2007) Comparison of Assignment Methods for Simulation-Based Dynamic Equilibrium Traffic Assignment, Presented in $6^{\text {th }}$ Triennial Symposium on Transportation Analysis.

Mahut, M., Florian, M., Tremblay, N., Campbell, M., Patman, D., and Mcdaniel, Z. K. (2004). Calibration and Application of a Simulation-Based Dynamic Traffic Assignment Model. Transportation Research Record: Journal of the Transportation Research Board, pp.101-111.

Margiotta, R., Lomax, T., Hallenbeck, M., Dowling, R., Skabardonis, A., and Turner, S. (2013). Analytical procedures for determining the impacts of reliability mitigation strategies. 92 $2^{\text {th }}$ Annual Meeting of the Transportation Research Board, National Research Council, Washington D.C., USA, No. SHRP 2 Report S2-L03-RR-1.

Martin, P., Zlatkovic, M., Chaudhuri, P., and Tasic, A. (2011). PTV Traffic Platform. Research Prepared by Civil and Environmental Engineering University of Utah.

Murray, P.M., Mahmassani, H.S., and Abdelghany, K.F. (2001)."Methodology for Assessing High-Occupancy Toll-Lane Usage and Network Performance. Transportation Research Record: Journal of the transportation Research Board, No.1765, pp. 8-15.

Nava, E., Milster, K., Chiu, Y.C., (2013). Modeling Managed Lane/Express Lane with Dynamic Traffic Assignment, Submitted for presentation at the 93nd Transportation Research Board Annual Meeting, Washington D.C.

Ngoduy, D. (2013). Instability of Cooperative Adaptive Cruise Control Traffic Flow: a Macroscopic Approach. In Communication Nonlinear Science and Numerical Simulation, Vol. 18, Issue 10, pp.2838-2851.

Koppelman, F., and Stats, G. (2013). Improving Our Understanding of How Highway Congestion and Pricing Affect Travel Demand. 92 $2^{\text {th }}$ Annual Meeting of the Transportation Research Board, National Research Council, Washington D.C., USA.

Parsons Brinckerhoff, (2013). Managed Lane Modeling Application for FSUTMS, Phase II, Memorandum to Advanced Traffic Assignment Subcommittee. Florida Department of Transportation, http://www.fsutmsonline.net/images/uploads/ManagedLane_Phase_II_Dr aft_2_2.docx, Accessed Oct. 23, 2013.

PTV Vision. (2013). VISUM .14, User Manual.

PTV Vision. (2013). VISSIM 7, User Manual. 
PTV Vision. (2015). VISSIM 8, User Manual.

Pueboobpaphan, R., Liu, F., and van Arem, B. (2010). The Impacts of a Communication based Merging Assistant on Traffic Flows of Manual and Equipped Vehicles at an OnRamp Using Traffic Flow Simulation. In Proceedings of the $13^{\text {th }}$ International IEEE Conference on Intelligent Transportation systems.

Rakha, H., and Mazen, A. (2010). Calibrating Steady-State Traffic Stream and CarFollowing Models Using Loop Detector Data. Transportation Science, Vol. 44, No. 2, pp. 151-168.

Reich, S. (2013). Automated and Autonomous Vehicles and Managed Lanes. Prepared for Tampa Hillsborough Expressway Authority, by Center for Urban Transportation Research, University of South Florida.

Resource Systems Group, (2012). South Florida Expressway Stated Preference Survey Report. Prepared by URS Corporation.

Rousseau, G., Scherr, W., Yuan, F., and Xiong, Ch. (2008). An Implementation Framework for Integrating Regional Planning Model with Microscopic Traffic Simulation. ASCE Journal of Transportation Engineering, pp. 3816-3825.

Ruegg, S., Kulshrestha, A., and Puppala, S. (2013). Phase I Managed Lane Assignment Model Testing I-95 Corridor, Orlando and Tampa Regional Model. Memorandum to Advanced Traffic Assignment Subcommittee, Florida Department of Transportation, http://www.fsutmsonline.net/images/uploads/ManagedLaneTesting_PhaseI.docx,Accesse d Oct. 20, 2013.

Sbayti, H., and Roden, D. (2010). Best Practices in the Use of Micro Simulation Models. NCHRP Project 8-36, Task 90, National Cooperative Highway Research Program, Washington, $D C$.

Safirova, E., Gillingham, K., Harrington, W., and Nelson, P. (2013). Resources for the Future, Are HOT Lanes a Hot Deal?. http://www.rff.org/rff/Documents/RFF-IB-0303.pdf, Accessed Oct. 20, 2013.

Scarinci, R., Hegyi, A., and Heydecker, B. G. (2013). Cooperative Ramp Metering; A Study of the Practicality of a Ramp Metering Development Using Intelligent Vehicles. Presented in 45 Annual UTSG Universities' Transport Study Group Conference.Shabanian, Sh. (2014). Advanced Methodologies in Dynamic Traffic Assignment Modeling of Managed Lanes. (Ph.D. Dissertation). Florida International University, Florida, United States.

Shladover, S., Su, D., and Lu, X. (2012). Impacts of Cooperative Adaptive Cruise Control on Freeway Traffic Flow. 91 ${ }^{\text {th }}$ Annual Meeting of the Transportation Research Board, National Research Council, Washington D.C., USA. 
Shelton, J., and Chiu, Y. (2009). Toward a Consistent and Robust Integrated MultiResolution Modeling Approach for Traffic Analysis. Transportation Research Board, Planning Application Conference. Houston, Texas.

SHRP 2 Report L38C. (2015). Testing of SHRP 2 Reliability Data and Analytical Products. https://trid.trb.org/view.aspx?id=1321186, Accessed Apr 2015.

SHRP 2 Report C04. (2013). Improving Our Understanding of How Highway CongestionandPricingAffectTravelDemand.http://onlinepubs.trb.org/onlinepubs/shrp $2 / S$ HRP2_S2-C04-RW-1.pdf, Accessed Apr 2013.

Tapani, A. (2012). Vehicle Trajectory Effects of Adaptive Cruise Control. In Journal of Intelligent Transportation Systems, Vol.16 (1), pp. 36-44.

Van Driel, C. J., and Van Arem, B. (2008) "Traffic Flow Impacts of a Congestion Assistant." $84^{\text {th }}$ Annual Meeting of the Transportation Research Board, National Research Council, Washington D.C., USA.

Van, A.B., and Van Der Vlist, M. (1991). An On-line Procedure for Estimating Current Capacity, Technical Report INRO-VVG 1991 -17, TNO Institute of Spatial Organization, Delft.

VISTA Transport Group, Inc. (2010). VISTA User Guide.

Wang, Y., Liu, X., Rouphail, N., Schroeder, B., Yin, Y., Bloomberg, L., and Santa Ana, C. A. (2012). Analysis of Managed Lanes on Freeway Facilities. Final Report for NCHRP Project 03-96. $91^{\text {th }}$ Annual Meeting of the Transportation Research Board, National Research Council, Washington D.C., USA.

Wang, J., and Rajamani, R. (2010). The Impact of Adaptive Cruise Control Systems on Highway Safety and Traffic Flow. Proceedings of the Institution of Mechanical Engineers, Part D: Journal of Automobile Engineering, Vol. 218, No. 2, pp. 111-130.

Xiao, Y., Fakharian Qom, S., Hadi, M., and Al-Deek, H. (2014). Use of Data from Point Detectors and Automatic Vehicle Identification to Compare Instantaneous and Experienced Travel Times. Transportation Research Record: Journal of the Transportation Research Board, No 2470, pp. 95-104.

Xiao, Y., Fakharian Qom, S., Hadi, M., and Al-Deek, H. (2014). Comparison of Instantaneous and Experienced Travel Time Using Point Detector Data and AVI Data. $84^{\text {th }}$ Annual Meeting of the Transportation Research Board, National Research Council, Washington D.C., USA.

Xiao, Y., Hadi, M., and Ozen, H. (2012). Development of a Data-Based Intelligent Transportation System Evaluation Tool. Prepared for Florida Department of Transportation, by the Florida International University Lehman Center for Transportation Research, Miami, Fl. 
Xiao, Y., Hadi, M., Ozen, H., and Mysore, V. (2010). An ITS Evaluation Tool in the FSUTMS Regional Demand Modeling Environment. Transportation Research Record: Journal of the Transportation Research Board, No. 2176, pp. 76-83.

Zhang, L., and Ma, J. (2012). A Study on Multi-Resolution Modeling of MesoscopicMicroscopic Traffic Simulation Model. ASCE Journal of Transportation Engineering, pp. 695-706.

Zhou, X., and Taylor, J. (2012). NEXTA/DTALite User Guide Visualization. http://code.ooglr.com/p/NEXTA/. Accessed September, 2012.

Ziliaskopoulos, A. K. (2000). A Linear Programming Model of Single Destination System Optimum Dynamic Traffic Assignment Problem. Transportation Science, 34(1): pp.37-49. 


\section{APPENDICES}

\section{APPENDIX 1. Review of Simulation and Dynamic Traffic Assignment (DTA) Tools VISUM}

Verkehr In Stadten Simulation Modeling, which means "Traffic in Towns Simulation Modeling" (VISUM) is a tool that allows modeling transportation systems and includes a DTA model that has been added to this software for the advanced modeling of the interaction between traffic path performance and route selection. The DTA model assigns dynamic Origin-Destination (O-D) matrices onto the network based on Dynamic User Equilibrium (DUE). The model converges to the equilibrium state in which no travelers can have less experienced travel time by unilaterally changing their paths (PTV Vision VISUM 14, 2013).

To represent a spillback in VISUM, it is assumed that each link is characterized by two time-varying bottlenecks: one located at the beginning, and another located at the end of the link, called "entry capacity" and "exit capacity", respectively. VISUM applies Traffic Flow Fuzzy (TFlowFuzzy) model to allow Origin-Destination Matrix Estimation (ODME) using observed count data and simulated volumes. The matrix estimation data is done using an iterative method to adjust the initial O-D matrix cells to achieve better matching of observed and simulated volumes (PTV Vision VISUM 14, 2013).

\section{DYNASMART}

Dynamic Network Assignment-Simulation Model for Advanced Telematics (DYNASMART) is one of the first DTA tools developed to implement a simulation-DTA 
modeling of transportation networks by Mahmassani et al. (2009). DYNASMART provides a mesoscopic level of traffic representation, which combines a microscopic level of representation of individual travelers with a macroscopic description of traffic flow. Link travels and movements of vehicles are governed by a modified version of the Greenshield's macroscopic speed-density relationship, but vehicular movements are tracked at the level of individual vehicles or groups of vehicles. Delay is computed using node transfer logic based on the time that takes for vehicles to transfer. The model assumes that O-D demands and departure times are provided. DYNASMART was initially tested on the Fort Worth network to evaluate real-time route guidance for incident and non-incident conditions under varying degrees of information supplied to different user classes. Additionally, DYNASMART was tested in the early stage of development using the Baltimore and Irvine network. Recently, DYNASMART was successfully used as a research tool for assessment, and used also as a part of advanced strategy implementations. This tool is not currently commercially available.

\section{DynusT}

DYNamic Urban Systems for Transportation (DynusT) was developed at the University of Arizona based on DYNASMART. DynusT is an open source program that was developed by Chiu (2012).

The default assignment in DynusT is based on a gap-based assignment which replaces the Method of Successive Average (MSA) assignment in a recent version of DynusT although the MSA assignment can still be requested. The gap-based assignment produces much better convergence and computational efficiency compared to MSA (Chiu 
and Bustillos, 2009). DynusT is an open-source tool and is not commercially available, although the developers can sign agreement to provide technical support. It has been used in a number of projects in recent years.

\section{VISTA}

The Visual Interactive System for Transportation Algorithms (VISTA) tool is a DTA-based software operated by VISTA Transport Group, Inc. (VTG) VISTA iterates between two modules, which are Path Generation (PG) and Dynamic User Equilibrium (DUE). In the first iteration, PG finds the time-dependent shortest path between each O-D pair at each departure time, assigns all vehicles to their shortest paths, and then simulates the vehicle movements to update travel costs. In subsequent iterations of PG, a fixed percentage of vehicles (as opposed to all vehicles) are allowed to move to the shortest paths before simulation. The DUE finds the optimal percentage of vehicles to be shifted from every other path onto the current shortest path. It then simulates these new vehicle trajectories to find new path costs and the new shortest path set. Convergence is measured after exercising both the PG and DUE modules by comparing the travel times across all vehicles with the same origin, destination, and departure time. VISTA VISTA has the ability to incorporate traffic signals, intersection movements, signal optimization, variable message signs, and multiple vehicle types including fixed-route transit vehicles and incidents that temporarily reduce capacity. VISTA can be run from a command line or using the web-based graphical user interface, which includes a geographic information system editor for the purpose of visualizing the network and animating results. More

details on VISTA's framework can be found in Ziliaskopoulous (2000). This software 
was tested for Chicago, Austin, and Dallas/Fort Worth metropolitan planning regions networks. However, the application of this software is still limited, particularly in recent years.

\section{Dynameq}

Dynameq is a DTA software developed by INRO Consultants, Inc. Dynameq is a DUE-based model that iterates between finding time-dependent path flows and determining the corresponding path travel times. Vehicles are assigned to paths using the MSA, which assigns a decreasing fraction of vehicles to the shortest path in subsequent iterations. The fraction is equal to one divided by the current iteration number, so that in the first iteration, all vehicles are assigned to the shortest path. Half of all vehicles are assigned to the shortest path in the second iteration, and so on. The developers have also tested more efficient and better converging methods of assignment (Mahut et al., 2007). Although Dynameq is a DUE-based model and vehicles do not switch paths en-route, lane-changing decisions are made upon entering each new link. Modeling individual lanes has the advantage of explicitly modeling scenarios when certain types of vehicles are restricted from specific lanes such as high occupancy vehicle lanes. To improve computational efficiency and allow for regional-level modeling, Dynameq's behavioral rules are simplified relative to other microscopic simulators. These simplifications include not allowing vehicles to reconsider their lane choice. Also, the model is updated each time an event occurs, rather than at pre-defined time intervals. Thus, Dynameq may be considered as higher fidelity mesoscopic model. More information about Dynameq and its application can be found in Mahut et al. (2004) and Florian et al. (2008). 


\section{DynaMIT}

Dynamic Network Assignment for the Management of Information to Travelers (DynaMIT) is a DTA model developed at the Massachusetts Institute of Technology. It has an online version that obtains real-time traffic data and predicts network conditions and an offline version mainly used as a planning tool. DynaMIT has two major components including demand simulator and a supply simulator that interact with each other to estimate the state of the system. The demand simulator makes use of historical O-D matrices and generates travelers with certain socio-economic characteristics based on the actual population. Route choice models are then used based on historic travel times to assign a habitual travel behavior for each traveler. In the next step travelers adjust their routes or departure times from their habitual behavior in the presence of network information. This is accomplished by Probit or nested Logit models using path travel time and cost as trip attributes. The demand is then aggregated and an online calibration model based on an autoregressive process using the Kalman filtering approach adjusts the demands to match real time data. The demand matrices are then disaggregated into individual lists of drivers as in the initial step and are loaded onto the simulator. The supply simulator captures the behavior of the network using traffic flow models. Links are divided into smaller segments, and each segment is associated with a speed-density relationship and a queuing part. A deterministic queuing model produces the waiting times in the queues using the output and queuing capacities of the segment. Capacities and several other parameters used in the speed-density equations can be calibrated both off-line and online. The simulator then updates the speeds and densities by loading the demand on the network in a time-based manner. The model was successfully applied to 
small networks such as Southampton, Lower Westchester County, and Irvine to study various traffic-related problems. This tool can be considered as a research tool since it has not been made available to agencies. More information on DynaMIT can be found in Ben-Akiva et al. (2009).

\section{Cube Avenue}

Cube Avenue is a dynamic traffic assignment extension of Cube Voyager (Citilabs, 2013). It models traffic at more details than the Cube Voyager's Highway program which utilizes macroscopic models, and at less detail than microscopic models. With Cube Avenue, routes and flow rates change during the modeling period based on congestion. One of the strength of Cube Avenue for regions that use the Cube modeling environment is to apply the same data format and scripting language as Highway Cube Voyager. Using this scripting language also provides more flexibility in modeling approaches.

The assignment in Cube Avenue is based on user equilibrium utilizing the MSA method.

\section{TRANSIMS}

Transportation ANalysis and SIMulation System (TRANSIMS) is an open-source software developed at the Los Alamos National Laboratory to conduct transportation system analysis. It consists of four steps, one of which estimates demand by an activitybased model, which is not available in other assignment tools. TRANSIMS has been implemented for large networks such as Dallas and Portland; however, it requires an 
extensive amount of input data compared to other DTA models. More information about TRANSIMS can be found in Lee et al. (2014).

\section{DTALite}

DTALite is an open-source light-weight DTA package that has been developed by Zhou and Taylor (2012) and supported by FHWA to allow a rapid utilization of advanced dynamic traffic analysis capabilities. DTALite is a mesoscopic simulation-based DTA package that works in conjunction with the Network EXplorer for Traffic Analysis (NEXTA) graphical user interface. The DTALite tool aims to integrate modeling and visualization capabilities using a modularized design, the open-source suite of simulation plus visualization interface.

The traffic assignment and simulation modules in DTALite iterate to either capture day-to-day user response or find steady-state equilibrium conditions. Speed, volume and density measures at the network, specific links, and vehicle trajectories can be visualized using the NEXTA user interface (Zhou and Taylor, 2012).

DTALite is a link-based simulation with capacity constraints and it has been used recently in several pilot and research project sponsored by FHWA program (FHWA, 2013a). More information about NEXTA/DTALite can be found at https://code.google.com/p/NEXTA (DTALite, 2012).

\section{TransModeler}

TransModeler is a microscopic simulation-based traffic assignment tool offered by Caliper (Caliper Corporation, 2011). One of the interesting features of TransModeler 
is that it allows the network modeling based on the microscopic, mesoscopic, and/or macroscopic simulation level in the same run.

TransModeler applies different algorithms that are suitable for microscopic simulation-based DTA. TransModeler can handle large-scale networks based on the microscopic simulation-based DTA. Micro-level simulation provides a more accurate representation of traffic and management operations compared to mesoscopic modeling. As these models become more efficient, this increases their attractiveness. However, calibration microscopic simulation still requests significantly more time than mesoscopic models, especially when combined with DTA.

More information about TransModeler can be found at http://www.caliper.com/TransModeler/Simulation.htm (Caliper Corporation, 2011).

\section{VISSIM}

Verkehr In Stadten Simulation, which means "Traffic in Towns Simulation (VISSIM) was developed by PTV Group in Germany (PTV Vision, VISSIM 7, 2013). Most existing simulation models operate using link-node configurations. VISSIM is a detailed microscopic simulation tool that models vehicles at the 0.1 -second resolution level. VISSIM differs from these models and it utilizes a link-connector structure. This involves coding movement individually at each intersection, allowing for increased precision and flexibility in modeling traffic flow. Although this process has been simplified in more recent versions of the software, it is more complex than coding linknode models. VISSIM can display microscopic simulation results in 3D animations, including a feature that allows viewing from a selected driver's perspective. VISSIM has 
also a power programing extension that allows modelers to program advanced managements and pricing strategies that correspond to real-world advanced strategies. VISSIM recently released the new version of VISSIM 8 which allows the user to specify the demands based on predetermined turning movement or to utilize DTA between origins and destinations. In addition, the tool has a ML model to estimate the diversion between ML and General Purpose Lanes (GPL). (PTV Vision, VISSIM 8, 2015). 
APPENDIX 2. Summary of Criteria for Tool Assessment

\begin{tabular}{|c|c|c|c|c|c|c|c|c|c|c|}
\hline Criterion & Cube Voyger & ELToD & VISUM & DTALite & DynsuT & Dynameq & Cube Avenue & VISSIM & $\begin{array}{c}\text { Trans } \\
\text { Modeler }\end{array}$ & CORSIM \\
\hline \multicolumn{11}{|c|}{ General Criteria (Hardware, Software, Interface, and etc) } \\
\hline Open Source & No & Yes & No & Yes & Yes & No & No & No & No & No \\
\hline $\begin{array}{c}\text { Utilization of } \\
\text { Additional Hardware } \\
\text { Computational } \\
\text { Capabilities }\end{array}$ & None & $\begin{array}{l}\text { Requires } \\
\text { Cube } \\
\text { Voyager }\end{array}$ & None & None & None & None & $\begin{array}{c}\text { Requires Cube } \\
\text { Voyager }\end{array}$ & None & None & None \\
\hline $\begin{array}{c}\text { Flexibility in } \\
\text { Modifying Procedures }\end{array}$ & $\begin{array}{c}\text { Cube scripting } \\
\text { language can be } \\
\text { modified }\end{array}$ & $\begin{array}{c}\text { Implementati } \\
\text { on of Cube } \\
\text { Voyager }\end{array}$ & $\begin{array}{c}\text { Can use API utilizing } \\
\text { Python script } \\
\text { language }\end{array}$ & $\begin{array}{c}\text { Open source. } \\
\text { Codes can be } \\
\text { modified. But } \\
\text { difficult to } \\
\text { modify. }\end{array}$ & $\begin{array}{l}\text { Open source. } \\
\text { Codes can be } \\
\text { modified }\end{array}$ & $\begin{array}{c}\text { Using a } \\
\text { Python-based } \\
\text { API } \\
\text { to implement } \\
\text { advanced } \\
\text { strategies }\end{array}$ & $\begin{array}{c}\text { Cube scripting } \\
\text { language can be } \\
\text { modified }\end{array}$ & \begin{tabular}{|} 
Can use API \\
utilizing \\
Python script \\
language
\end{tabular} & $\begin{array}{c}\text { Can use } \\
\text { script } \\
\text { language or } \\
\text { API to } \\
\text { implement } \\
\text { advanced } \\
\text { strategies }\end{array}$ & $\begin{array}{l}\text { The Real } \\
\text { Time } \\
\text { Extension } \\
\text { (RTE) } \\
\text { facility } \\
\text { allows } \\
\text { coding of } \\
\text { advanced } \\
\text { strategies }\end{array}$ \\
\hline $\begin{array}{c}\text { User } \\
\text { Interface/Software } \\
\text { Interface }\end{array}$ & $\begin{array}{l}\text { Make use of } \\
\text { Cube } \\
\text { environment } \\
\text { powerful } \\
\text { interface }\end{array}$ & $\begin{array}{c}\text { Implementati } \\
\text { on of Cube } \\
\text { Voyager }\end{array}$ & $\begin{array}{c}\text { State of the art } \\
\text { interface. There is no } \\
\text { interface with other } \\
\text { tools }\end{array}$ & \begin{tabular}{|} 
Use the \\
NEXTA \\
interface, \\
which is a \\
user friendly \\
interface. \\
Data in \\
NEXTA can \\
be converted \\
to Cube and \\
VISSIM
\end{tabular} & $\begin{array}{c}\text { Use the } \\
\text { NEXTA } \\
\text { interface. The } \\
\text { interface has } \\
\text { been developed } \\
\text { to convert } \\
\text { DynusT files to } \\
\text { VISUM, which } \\
\text { can then be } \\
\text { converted to } \\
\text { VISSIM }\end{array}$ & $\begin{array}{l}\text { Similar in } \\
\text { design to the } \\
\text { Emme } 4 \\
\text { interface }\end{array}$ & $\begin{array}{l}\text { Make use of } \\
\text { Cube } \\
\text { environment } \\
\text { powerful } \\
\text { interface }\end{array}$ & $\begin{array}{c}\text { State of the } \\
\text { art interface. } \\
\text { There is no } \\
\text { interface with } \\
\text { other tools }\end{array}$ & $\begin{array}{l}\text { State of the } \\
\text { art. } \\
\text { Interfaced } \\
\text { with } \\
\text { TransCAD }\end{array}$ & $\begin{array}{l}\text { Good user } \\
\text { interface } \\
\text { but limited } \\
\text { interface } \\
\text { with other } \\
\text { software }\end{array}$ \\
\hline
\end{tabular}




\begin{tabular}{|c|c|c|c|c|c|c|c|c|c|c|}
\hline Criterion & Cube Voyger & ELToD & VISUM & DTALite & DynsuT & Dynameq & Cube Avenue & VISSIM & $\begin{array}{c}\text { Trans } \\
\text { Modeler }\end{array}$ & CORSIM \\
\hline \multicolumn{11}{|c|}{ Shortest Path and Path Choice } \\
\hline Assignment Type & $\begin{array}{l}\text { Static user } \\
\text { equilibrium } \\
\text { (UE). Several } \\
\text { algorithms } \\
\text { available } \\
\text { including } \\
\text { 1. Bi-Conjugate } \\
\text { Frank-Wolf } \\
\text { method, path- } \\
\text { based gradient } \\
\text { projection } \\
\text { assignment } \\
\text { 2. Gradient } \\
\text { Projection } \\
\text { Algorithm, } \\
\text { origin based } \\
\text { 3. Junction } \\
\text { based }\end{array}$ & $\begin{array}{l} \\
\text { Use the static } \\
\text { user } \\
\text { equilibrium } \\
\text { in Cube } \\
\text { Voyager } \\
\\
\end{array}$ & \begin{tabular}{|c|} 
Use different types of \\
static and dynamic \\
assignment including: \\
Static : \\
1. Incremental \\
assignment \\
2. Equilibrium \\
assignment \\
3. Equilibrium Lohse \\
assignment(Time \\
Based User \\
Equilibrium) \\
4. Assignment with \\
Intersection Capacity \\
Analysis (ICA) \\
Stochastic assignment \\
5. TRIBUT (Time \\
Based User \\
Equilibrium) \\
Dynamic : \\
1. TRIBUT \\
Equilibrium (Time \\
Based User \\
Equilibrium) \\
2. Dynamic User \\
Equilibrium (DUE) \\
3. Dynamic stochastic \\
assignment \\
4. Equilibrium \\
assignment , Linear \\
User Cost Equilibrium \\
(LUCE)
\end{tabular} & \begin{tabular}{|c|} 
\\
\\
\\
Dynamic user \\
equilibrium \\
(DUE). \\
Alternative \\
methods are \\
available \\
including \\
MSA, Fix \\
Switching \\
Rate (FSR), \\
Day-to-Day \\
learning, and \\
OD Matrix \\
Estimation \\
(ODME) \\
\\
\end{tabular} & $\begin{array}{l}\text { MSA and } \\
\text { recently } \\
\text { introduced } \\
\text { GFV-based } \\
\text { method that } \\
\text { performs } \\
\text { significantly } \\
\text { better }\end{array}$ & $\begin{array}{c}\begin{array}{c}\text { Dynamic user } \\
\text { equilibrium } \\
\text { (DUE) }\end{array} \\
\text { 1. Fastest path } \\
\text { combined with } \\
\text { regular MSA } \\
\text { 2.flow } \\
\text { balancing } \\
\text { MSA } \\
\text { Gradient-Like } \\
\text { algorithm }\end{array}$ & $\begin{array}{l}\text { Dynamic user } \\
\text { equilibrium } \\
\text { (DUE) utilizing } \\
\text { MSA }\end{array}$ & $\begin{array}{l}\text { Dynamic user } \\
\text { equilibrium } \\
\text { (DUE). }\end{array}$ & $\mid \begin{array}{c}\text { Dynamic } \\
\text { user } \\
\text { equilibrium } \\
\text { (DUE). }\end{array}$ & $\begin{array}{c}\text { Use a static } \\
\text { assignment } \\
\text { before the } \\
\text { simulation } \\
\text { but this } \\
\text { feature is } \\
\text { not } \\
\text { normally } \\
\text { used. }\end{array}$ \\
\hline
\end{tabular}




\begin{tabular}{|c|c|c|c|c|c|c|c|c|c|c|}
\hline Criterion & Cube Voyger & ELToD & VISUM & DTALite & DynsuT & Dynameq & Cube Avenue & VISSIM & $\begin{array}{c}\text { Trans } \\
\text { Modeler }\end{array}$ & CORSIM \\
\hline $\begin{array}{c}\text { En-route Dynamic } \\
\text { Routing (e.g., in- } \\
\text { vehicle dynamic } \\
\text { navigation system, } \\
\text { DMS) }\end{array}$ & No & No & No & $\begin{array}{c}\text { Yes. } \\
\text { In-vehicle } \\
\text { and DMS }{ }^{1} \text { ) }\end{array}$ & $\begin{array}{c}\text { Yes. } \\
\text { In-vehicle and } \\
\text { DMS }\end{array}$ & No & No & $\begin{array}{l}\text { No. } \\
\text { But divert at } \\
\text { DMS using } \\
\text { API }\end{array}$ & Yes & No \\
\hline $\begin{array}{c}\text { Specification of Fine- } \\
\text { Grained Assignment } \\
\text { Interval (e.g., 15-30 } \\
\text { minutes) }\end{array}$ & No & No & Yes & Yes & Yes & Yes & Yes & Yes & Yes & Yes. \\
\hline $\begin{array}{l}\text { Allows Fixing Paths } \\
\text { for Parts of the } \\
\text { Demands }\end{array}$ & $\begin{array}{c}\text { No. } \\
\text { But can be } \\
\text { emulated by } \\
\text { restricting the } \\
\text { link in the path. } \\
\text { For specific } \\
\text { demands } \\
\text { (maybe difficult } \\
\text { in some cases) }\end{array}$ & \begin{tabular}{|c|} 
No. \\
But can be \\
emulated by \\
restricting the \\
link in the \\
path. For \\
specific \\
demands \\
(maybe \\
difficult in \\
some cases)
\end{tabular} & Yes & Yes & Yes & Yes & $\begin{array}{c}\text { No. } \\
\text { But can be } \\
\text { emulated } \\
\text { restricting the } \\
\text { link in the path. } \\
\text { For specific } \\
\text { demands (maybe } \\
\text { difficult in some } \\
\text { cases) }\end{array}$ & Yes & Yes & No \\
\hline Convergence Criteria & Link based & Link based & Trip based & Trip based & Trip based & Trip based & Link based & \begin{tabular}{|c|} 
Convergence \\
in the form of \\
the ' $\mathrm{P}^{2}$, and \\
' $\mathrm{V}^{3}$, \\
statisticsi
\end{tabular} & $\begin{array}{c}\text { DUE }^{4} \\
\text { relative gap }\end{array}$ & N/A \\
\hline
\end{tabular}

${ }^{1}$ DMS: Dynamic Massage Sign

2 ' $\mathrm{P}$ ': The percentage of links on which flows change by less than $5 \%$ between successive iterations ,,

${ }^{3}$ ' $\mathrm{V}$ ': The percentage change in total user costs or time spent in the network between successive iterations

${ }^{4}$ DUE: Dynamic User Equilibrium 


\begin{tabular}{|c|c|c|c|c|c|c|c|c|c|c|}
\hline Criterion & Cube Voyger & ELToD & VISUM & DTALite & DynsuT & Dynameq & Cube Avenue & VISSIM & $\begin{array}{c}\text { Trans } \\
\text { Modeler }\end{array}$ & CORSIM \\
\hline $\begin{array}{c}\text { Outputting and Using } \\
\text { Interval-based } \\
\text { Convergence Gap }\end{array}$ & N/A & N/A & $\begin{array}{l}\text { Utilizes gap for the } \\
\text { whole iteration. }\end{array}$ & $\begin{array}{c}\text { Utilizes gap } \\
\text { for the whole } \\
\text { iteration }\end{array}$ & $\begin{array}{l}\text { Utilizes gap for } \\
\text { the whole } \\
\text { iteration. }\end{array}$ & $\begin{array}{l}\text { Utilizes gap } \\
\text { for each } \\
\text { interval. }\end{array}$ & $\begin{array}{l}\text { Utilizes gap for } \\
\text { the whole } \\
\text { iteration. }\end{array}$ & NA & $\begin{array}{c}\text { Interval- } \\
\text { based } \\
\text { relative } \\
\text { gaps and } \\
\text { uses only } \\
\text { the average } \\
\text { gap }\end{array}$ & N/A \\
\hline $\begin{array}{c}\text { Assignment of } \\
\text { Individual Vehicles }\end{array}$ & No & No & No & Yes & Yes & Yes & No & Yes & Yes & N/A \\
\hline $\begin{array}{l}\text { Assignment of } \\
\text { Multiple Demand } \\
\text { Types }\end{array}$ & $\begin{array}{c}\text { Yes. } \\
\text { Different user } \\
\text { classes for each } \\
\text { demand type } \\
\text { defined by user }\end{array}$ & \begin{tabular}{|c} 
Yes. \\
Different user \\
classes for \\
each demand \\
type defined \\
by user
\end{tabular} & \begin{tabular}{|c|} 
Yes. \\
Based on each user \\
classes, by \\
considering each \\
demand type as type \\
of PrT (Private \\
Transpiration) system \\
in link attribute \\
\end{tabular} & \begin{tabular}{|c|} 
Yes. \\
Allows only \\
three \\
different \\
types of \\
users: SOV, \\
HOV, and \\
Truck. \\
However, the \\
value of time \\
can be \\
specified as a \\
random \\
variable for \\
each user \\
type
\end{tabular} & \begin{tabular}{|} 
Yes. \\
Allow only \\
three different \\
types of users: \\
SOV $^{1}, \mathrm{HOV}^{2}$, \\
and Truck \\
\end{tabular} & $\begin{array}{c}\text { Yes. } \\
\text { Different user } \\
\text { classes for } \\
\text { each demand } \\
\text { type defined } \\
\text { by user }\end{array}$ & $\begin{array}{c}\text { Yes. } \\
\text { Different user } \\
\text { classes for each } \\
\text { demand type } \\
\text { defined by user }\end{array}$ & $\begin{array}{l}\text { Yes. } \\
\text { Based on } \\
\text { each user } \\
\text { classes, by } \\
\text { considering } \\
\text { each demand } \\
\text { type in link } \\
\text { attribute }\end{array}$ & $\begin{array}{l}\text { Yes. } \\
\text { Based on } \\
\text { vehicle } \\
\text { class, or } \\
\text { driver } \\
\text { group }\end{array}$ & N/A \\
\hline
\end{tabular}

${ }^{1}$ SOV: Single Occupancy Vehicle

${ }^{2}$ HOV: High Occupancy Vehicle 


\begin{tabular}{|c|c|c|c|c|c|c|c|c|c|c|}
\hline Criterion & Cube Voyger & ELToD & VISUM & DTALite & DynsuT & Dynameq & Cube Avenue & VISSIM & $\begin{array}{c}\text { Trans } \\
\text { Modeler }\end{array}$ & CORSIM \\
\hline \multicolumn{11}{|c|}{ Traffic Flow Model (TFM) } \\
\hline $\begin{array}{c}\text { Traffic Flow Model } \\
\text { Type }\end{array}$ & Macroscopic & Macroscopic & Macroscopic & Mesoscopic & Mesoscopic & Msoscopic & Mesoscopic & Microscopic & $\mid \begin{array}{c}\text { Microscopi } \\
\text { c }\end{array}$ & Microscopc \\
\hline $\begin{array}{l}\text { Queuing and } \\
\text { Spillback }\end{array}$ & No & No & Yes & Yes & Yes & Yes & Yes & Yes & Yes & Yes \\
\hline $\begin{array}{c}\text { Modeling of } \\
\text { Signalized Arterials }\end{array}$ & Yes & No & Yes & Yes & Yes & Yes & Yes & Yes & Yes & Yes \\
\hline Modeling of Freeways & Yes & Yes & Yes & Yes & Yes & Yes & Yes & Yes & Yes & Yes \\
\hline $\begin{array}{c}\text { Modeling of } \\
\text { Alternative Routes to } \\
\text { Facilities }\end{array}$ & Yes & $\begin{array}{c}\text { No. } \\
\text { Only ML and } \\
\text { GPL }\end{array}$ & Yes & Yes & Yes & Yes & Yes & Yes & Yes & Yes \\
\hline $\begin{array}{c}\text { Automatic } \\
\text { Calculation of Signal } \\
\text { Timing in Dynamic } \\
\text { Traffic Assignment }\end{array}$ & Yes & No & Yes & Yes & Yes & Yes & Yes & No & Yes & NA \\
\hline $\begin{array}{l}\text { Lane-by-Lane } \\
\text { Simulation }\end{array}$ & No & No & No & No & No & Yes & No & Yes & Yes & Yes \\
\hline $\begin{array}{l}\text { Merging/Weaving } \\
\text { Simulation }\end{array}$ & No & No & No & No & No & Yes & No & Yes & Yes & Yes \\
\hline $\begin{array}{l}\text { Modeling Turn Lane } \\
\text { and Bay Length }\end{array}$ & No & No & No & No & No & Yes & No & Yes & Yes & Yes \\
\hline
\end{tabular}




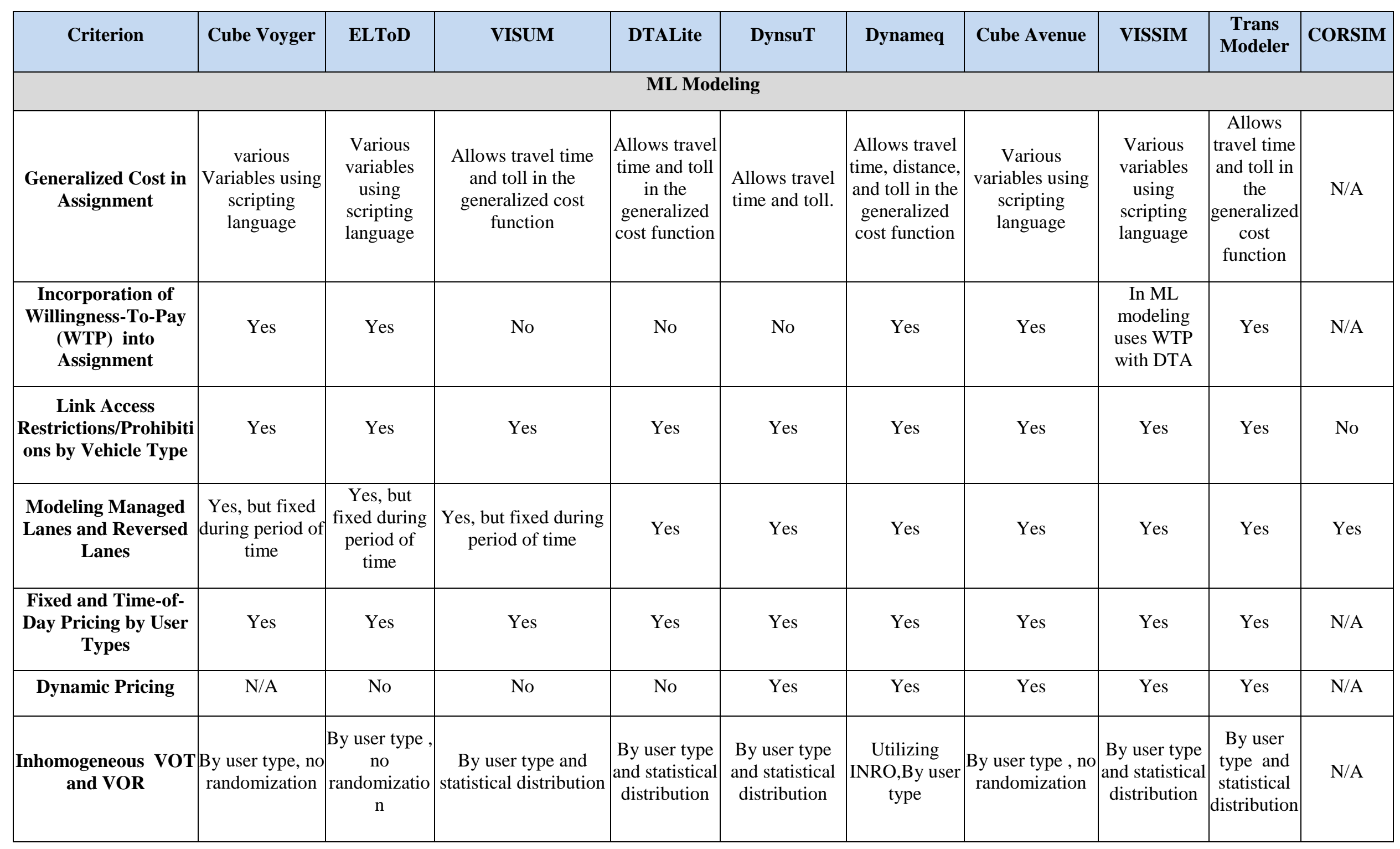




\begin{tabular}{|c|c|c|c|c|c|c|c|c|c|c|}
\hline Criterion & Cube Voyger & ELToD & VISUM & DTALite & DynsuT & Dynameq & Cube Avenue & VISSIM & $\begin{array}{c}\text { Trans } \\
\text { Modeler }\end{array}$ & CORSIM \\
\hline \multicolumn{11}{|c|}{ Advanced Vehicle Technology } \\
\hline $\begin{array}{c}\text { Capacity as a } \\
\text { Function of } \\
\text { Proportion of Vehicle } \\
\text { Types }\end{array}$ & Yes & $\begin{array}{c}\text { Maybe } \\
\text { emulated by } \\
\text { coding PC } \\
\text { and trucks }\end{array}$ & $\begin{array}{l}\text { Maybe emulated by } \\
\text { coding PC and trucks }\end{array}$ & $\begin{array}{l}\text { Maybe } \\
\text { emulated by } \\
\text { coding PC } \\
\text { and trucks }\end{array}$ & $\begin{array}{c}\text { Maybe } \\
\text { emulated by } \\
\text { coding PC and } \\
\text { trucks }\end{array}$ & Yes & Yes & Yes & $\begin{array}{l}\text { Can be } \\
\text { emulated } \\
\text { by coding } \\
\text { PC and } \\
\text { trucks }\end{array}$ & $\begin{array}{c}\text { Maybe } \\
\text { emulated } \\
\text { by coding } \\
\text { PC and } \\
\text { trucks }\end{array}$ \\
\hline $\begin{array}{c}\text { Fixed and Time-of- } \\
\text { Day Pricing by } \\
\text { different percentage } \\
\text { of Advanced Vehicle } \\
\text { Technology }\end{array}$ & Yes & $\begin{array}{l}\text { Maybe } \\
\text { emulated by } \\
\text { coding PC } \\
\text { and trucks }\end{array}$ & $\begin{array}{l}\text { Maybe emulated by } \\
\text { coding PC and trucks }\end{array}$ & $\begin{array}{l}\text { Maybe } \\
\text { emulated by } \\
\text { coding PC } \\
\text { and trucks }\end{array}$ & $\begin{array}{c}\text { Maybe } \\
\text { emulated by } \\
\text { coding PC and } \\
\text { trucks }\end{array}$ & Yes & Yes & Yes & Yes & $\begin{array}{c}\text { Maybe } \\
\text { emulated } \\
\text { by coding } \\
\text { PC and } \\
\text { trucks }\end{array}$ \\
\hline
\end{tabular}

${ }^{1}$ PC: Passenger Car 
VITA

\section{SOMAYE FAKHARIAN QOM}

\section{EDUCATION AND AWARDS}

$\begin{array}{ll}2002-2006 & \text { B.S., Civil Engineering } \\ & \text { Iran University of Science and Technology, Tehran, Iran } \\ 2007-2009 & \text { M.S., Graduate Assistant } \\ & \text { Civil Engineering - Transportation } \\ & \text { Iran University of Science and Technology, Tehran, Iran } \\ 2013-2015 & \text { Doctoral Candidate and Graduate Assistant } \\ & \text { Civil Engineering - Transportation } \\ & \text { Florida International University, Miami, Florida } \\ 2015-2016 & \text { Doctoral Candidate and Dissertation Year Fellow (DYF) } \\ & \text { Civil Engineering - Transportation } \\ & \text { Florida International University, Miami, Florida }\end{array}$

Outstanding World Ahead Graduate, Office of Provost, Florida International University, Summer 2016.

Outstanding Ph.D. Graduate Award, College of Engineering, Florida International University, Summer 2016.

Recipient of $1^{\text {st }}$ Place of Top Student Paper, International Award, Transportation Research Board (TRB), $15^{\text {th }}$ International Conference on Managed Lane (ML), May 2016.

Candidate to Participate in the Best Ph.D. Student Research in Transportation Operations and Traffic Control session, Transportation Research Board (TRB), January 2016.

Outstanding Dissertation Year Fellowship (DYF), Florida International University, Spring and Summer 2016.

Recipient of $3^{\text {rd }}$ Place of Student Essay Competition, National Award, Intelligent Transportation System (ITS) America, June 2015.

Graduate \& Professional Student Committee (GPSC) Conference Funding Award, Graduate School, Florida International University, March 2015 and January 2016 
Anne S. Brewer Scholarship, Outstanding Scholastic Achievement, Intelligent Transportation Society (ITS) of Florida, December 2014.

Dwight David Eisenhower, Transportation Fellowship Award, U.S. Department of Transportation, Federal Highway Administration (FHWA), Academic Year 2013-2014.

Graduate Assistantship, Florida International University, Lehman Center for Transportation Research, Spring 2013 through Spring 2016.

Outstanding Leadership Award, President of Women in Transportation Seminars (WTS), Florida International University, 2013-2015.

Co-Founder and President, WTS Student Chapter at FIU

2013- 2015

Transportation Research Board (Reviewer)

Intelligent Transportation Systems (ITS) America, (Member)

2014-Present

Institute of Transportation engineers (ITE), (Member)

2015-Present

Women's Transportation Seminars (WTS), (Member)

2013-Present

American Society of Civil Engineers (ASCE), (Member)

2013-Present

2013-Present

\section{JOURNAL PUBLICATIONS}

Fakharian Qom, S., Xiao, Y., and Hadi, M. "Evaluation of Cooperative Adaptive Cruise Control (CACC) Vehicles on Managed Lanes Utilizing Macroscopic and Mesoscopic Simulation", Transportation Research Record: Journal of the Transportation Research Board, (In press).

Fakharian Qom, S., Amini, A.A., Moghadasnejad, F., and Fazaeli, H. "Analysis of Crash Injury Severity for Interstate Roads Utilizing Crash Data and Traffic Flow Data", Journal of Transportation Engineering, ASCE, (In press).

Fakharian Qom, S., Xiao, Y., Hadi, M., and Hadi, M. Al-Deek, H. "Estimation of Queue Length for Freeway Facilities Based on the Combination of Point Traffic Detector Data and Automatic Vehicle Identification Data", Journal of Advanced Transportation, (Under Review)

Fakharian Qom, S., Xiao, Y., Hadi, M., and Al-Deek, H. (2015). "Estimation of Freeway Density Based on the Combination of Point Traffic Detector Data and Automatic Vehicle Identification Data", Transportation Research Record: No.2484, Journal of the Transportation Research Becord, pp. 110-118.

Xiao, Y., Fakharian Qom, S., Hadi, M., and Al-Deek, H. (2014). "Use of Data from Point Detectors and Automatic Vehicle Identification to Compare Instantaneous and Experienced Travel Times", Transportation Research Record: No.2470, Journal of the Transportation Research Board, pp. 95-104. 